\title{
Aspects of slice stability in Locale Theory
}

\author{
C. F. Townsend
}

July 2008

\begin{abstract}
It is shown that a particular categorical axiomatisation of the category of locales is slice stable. This localic slice stability can be used to recover the fundamental theorem of topos theory. A categorical account of the ideal completion of a preorder is developed and is used to give a new proof of Joyal and Tierney's result on the slice stability of locales.

Keywords: locale, power monad, ideal completion, sheaf, local homeomorphism, relational composition.

MSC 2000 Classification: 06D22; 18B25, 18B10, 18D20, $18 \mathrm{~F} 15$.
\end{abstract}

\section{Contents}

1 Introduction 3

1.1 Outline contents .................. 4

2 Categorical background $\mathbf{5}$

2.1 Order enriched categories . . . . . . . . . . . . 5

2.2 Categorical change of base . . . . . . . . . . . 6

$\begin{array}{llr}3 & \text { The axioms } & 8\end{array}$

3.1 Basic axioms . . . . . . . . . . . . . . 8

3.2 The double power object axiom . . . . . . . . . . . . . . 13

3.3 The upper and lower power monad axiom . . . . . . . . . . . . 14

4 Properties of discrete objects in $\mathcal{C} \quad \mathbf{1 6}$

4.1 Open maps . . . . . . . . . . . . . . . . . . . 17

4.2 Application of the Hofmann-Mislove theorem to exponentiability 20

4.3 Relational composition . . . . . . . . . . . . . . . 23

4.4 The ideal completion of a preorder . . . . . . . . . . . 26

4.5 The ideal completion of a join semilattice . . . . . . . . . . . 32

5 Applications $\quad \mathbf{3 7}$

5.1 The fundamental theorem of topos theory . . . . . . . . . . . 37

5.2 Localic slice stability . . . . . . . . . . . . . . . . . 39

6 Concluding comments $\quad 45$ 
7 Appendix

7.1 Product as join semilattice tensor . . . . . . . . . . . . . . 46

8 References

50 


\section{Introduction}

An axiom, placed on a category $\mathcal{C}$, is said to be slice stable if whenever it is satisfied by $\mathcal{C}$ it is also satisfied by the slice category $\mathcal{C} / X$ for every object $X$ of $\mathcal{C}$. The easiest example is the axiom of having finite limits (i.e. being cartesian); if $\mathcal{C}$ has finite limits then so too does $\mathcal{C} / X$ for any object $X$ of $\mathcal{C}$. A non-example is the axiom of having finite products since there are examples of categories that have finite products but not equalizers. The fundamental theorem of topos theory, e.g. A2.3 of [J02], is the assertion that the axioms of an elementary topos are slice stable.

The category of locales, Loc, enjoys the property,

$$
\operatorname{Loc}_{S h(Y)} \simeq \operatorname{Loc} / Y
$$

for any locale $Y$, where $\operatorname{Sh}(Y)$ is the topos of sheaves over $Y$, [JT84]. So any fact about the category of locales that can be proved within a topos is true of any slice of the category of locales. Since most of the results of locale theory are provable using only intuitionistic logic and so can be proved in any topos, this applies to most of locale theory. From this we intuitively know that the category of locales is slice stable ('logically' slice stable say). Slice stability in the literature invariably refers to this logical slice stability. However what is missing is a set of axioms for the category of locales which is slice stable. Whilst axiomatic approaches have been developed (see [T04], [T05] or [T06]; and also [V95] and [Tay00] for not unrelated techniques) these approaches are not shown to be slice stable. The main result of this paper is to show that the axiomatic accounts of locale theory developed in [T04], [T05] and [T06] are all slice stable with only trivial modifications (these trivial modifications do not effect the results available and so do not constitute a weakening of the theory).

Two applications are given. Firstly we reprove the fundamental theorem of topos theory as a consequence of this slice stability. Secondly we exploit slice stability to reprove $\operatorname{Loc}_{S h(Y)} \simeq \mathbf{L o c} / Y$ so showing that the familiar logical slice stability of locale theory is an aspect of axiomatic slice stability. We are able to prove this second result with no significant use of topos theoretic machinery as we do not have to develop set theoretic constructions internal to a topos of sheaves. However we will assume familiarity with the well understood relationship

$$
\operatorname{Sh}(Y) \simeq \mathbf{L H} / Y
$$

that is central to sheaf theory (here $\mathbf{L H} / Y$ is the category of local homeomorphisms over $Y$ ). The hope therefore is to make Joyal and Tierney's result on the slice stability of locales more accessible to sheaf theorists (albeit at the expense of a fairly involved discussion of various categorical aspects of locale theory).

This paper does not focus on the relationship between the categorical axioms discussed and their canonical model (the category locales). Therefore it does not provide much detail on the general topological motivation that underpins the work; however such motivation can be found in the literature, e.g. [J82] and [Vic 89]. For motivation on categorical approaches to topology/locale theory consult for example [V95] and [Tay00]. 


\subsection{Outline contents}

We begin by making some comments on lattices internal to order enriched categories and recalling categorical change of base. This change of base technique can be described under mild categorical assumptions and is central to nearly all of the proofs in the rest of the paper.

Next we recall the axioms to be discussed and show that they are slice stable. The key technical result is a proof that the axiomatic representation of the double coverage theorem (introduced in [VT04]) is slice stable. The discussion then focuses on the power monads and we show that certain properties of these monads are, in the presence of the other axioms, slice stable. This allows us to conclude that all of the results of [T04] and [T05] are slice stable.

In particular we can then conclude that the Hofmann-Mislove theorem (a categorical account of which is the main result of [T05]) is slice stable. As an application of this we prove axiomatically the known result that discrete objects are exponentiable and show how the isomorphism implicit in this observation can be described using relational composition. With this categorical description of relational composition we are able to introduce axiomatically the ideal completion of a preorder. We check that this construction has the right properties and that it agrees with the more general construction given in [T05]. When acting on semilattices we show that the ideal completion construction is functorial. Although the results contained within this section are just basic aspects of lattice theory the section is long. This is because we are no longer working within set theory but are reliant on a categorical axiomatisation and so every step is a categorical manipulation rather than a more familiar set theoretic one.

The next section consists of applications. It gives two proofs using the techniques developed; firstly of the fundamental theorem of topos theory and secondly of the Joyal and Tierney result on slice stability (i.e. $\operatorname{Loc}_{S h(Y)} \simeq \operatorname{Loc} / Y$ ). The fundamental theorem of topos theory becomes a categorical triviality since every topos, $\mathcal{E}$, is equivalent to the category of discrete locales over $\mathcal{E}$.

The proof of $\operatorname{Loc}_{S h(Y)} \simeq \mathbf{L o c} / Y$ is a bit more involved. Firstly we note that the category $S h(Y)$ can be expressed axiomatically using local homeomorphisms in the usual manner. Next the proof centres on a verification that, in the slice over any locale $Y$, the ideal completion of local homeomorphisms (that are also internal semilattices) has a right adjoint which, in fact, is monadic. The proof is then completed with a verification that the induced monad is the downset monad whose algebras are exactly frames in $S h(Y)$.

The final section is a summary section which also contains a table of the axioms together with some comments on them, for example noting known dependencies. An appendix is included that contains a proof that a particular axiom (used only in [T06] and not used for the results of this paper) is slice stable. 


\section{Categorical background}

\subsection{Order enriched categories}

Our category $\mathcal{C}$, to be axiomatised, is order enriched. Let us start by making a few general comments about order enriched categories. Firstly it must be noted that when limits or any other universal constructions are discussed relative to an order enriched category it is assumed that they are order enriched; that is, establish order isomorphisms on the relevant partially ordered homsets rather than just bijections. For example if $\mathcal{C}$ has binary products then it is assumed that the function

$$
\mathcal{C}(Z, X \times Y) \stackrel{\left.\left(\pi_{1} \circ{ }_{-}\right), \pi_{2} \circ(-)\right)}{\longrightarrow} \mathcal{C}(Z, X) \times \mathcal{C}(Z, Y)
$$

is an order isomorphism and not just a bijection.

In an order enriched category with finite products we will invariably discuss order internal meet (or join) semilattices. These are just ordinary internal semilattices but with the further requirement that the join(meet) map is left(right) adjoint to the diagonal and that the unit is left(right) adjoint to the nullary diagonal (i.e. the unique map to 1 ). Therefore for an object $X$ in an order enriched category we have that there is at most one order enriched join or meet semilattice structure on it; this is by uniqueness of adjoints.

An order enriched functor $F: \mathcal{C} \rightarrow \mathcal{D}$ between order enriched categories $\mathcal{C}$ and $\mathcal{D}$ is just an ordinary functor but with the additional property that $F$, on morphisms, is monotone. To prove that $F$ preserves order internal join or meet semilattices it is sufficient to check that it preserves products. Note that for any particular order internal join or meet semilattice, $X$ say, it just needs to be checked that $F$ preserves $\Delta_{X}: X \rightarrow X \times X$ and $!^{X}: X \rightarrow 1$ in order to conclude that $F(X)$ is an order internal join or meet semilattice.

Let us comment on the presheaf categories of order enriched categories. Central to the axiomatic system to be discussed is the interaction between $\mathcal{C}$ and its presheaf category $\left[\mathcal{C}^{o p}, \mathbf{S e t}\right]$. However if $\mathcal{C}$ is order enriched then $\left[\mathcal{C}^{o p}, \mathbf{S e t}\right]$ does not have a natural ordering on all of its homsets. There are two ways round this problem: (i) develop the theory relative to the order enriched category $\left[\mathcal{C}^{o p}, \mathbf{P o s}\right]$ where Pos is the category of posets or (ii) note that in practice for all the presheaves that we are concerned with the homsets between them do in fact have a natural ordering and so are posets. The first option is viable and in fact all the axioms do hold with $\left[\mathcal{C}^{o p}, \mathbf{P o s}\right]$ in place of $\left[\mathcal{C}^{o p}\right.$, Set $]$. However we have chosen to stick with the more familiar $\left[\mathcal{C}^{o p}\right.$, Set] for this exposition as this avoids the need to re-check the axioms in the slightly different context of an order enriched presheaf category.

Finally we make some observations about internal semilattices in $\left[\mathcal{C}^{o p}\right.$, Set]. Say $X$ and $Y$ are objects of an order enriched cartesian category $\mathcal{C}$. Consider the presheaf

$$
\begin{aligned}
\mathcal{C}^{o p} & \rightarrow \text { Set } \\
Z & \longmapsto \mathcal{C}(Z \times Y, X) .
\end{aligned}
$$


The notation $X^{Y}$ is used for this presheaf since it can be verified that $X^{Y}$ is the exponential $\mathcal{C}\left({ }_{-}, X\right)^{\mathcal{C}(-, Y)}$ in the category $\left[\mathcal{C}^{o p}\right.$, Set]. If $X$ is an order internal join semilattice in $\mathcal{C}$ then $X^{Y}$ is an internal join semilattice in $\left[\mathcal{C}^{o p}\right.$, Set]. Now the homsets $\left[X^{Y}, X^{Y} \times X^{Y}\right],\left[X^{Y} \times X^{Y}, X^{Y}\right],\left[1, X^{Y}\right]$ and $\left[X^{Y}, 1\right]$ are all posets as they inherit an ordering from $\mathcal{C}$ and it can be seen that the binary and nullary join maps on $X^{Y}$ are left adjoint to the binary and nullary diagonal maps in $\left[X^{Y}, X^{Y} \times X^{Y}\right]$ and $\left[X^{Y}, 1\right]$ respectively. Therefore to prove that a functor $F:\left[\mathcal{C}^{o p}\right.$, Set $] \rightarrow\left[\mathcal{D}^{o p}\right.$, Set $]$ preserves the semilattice structure on $X^{Y}$ we have but to check that it preserves the ordering on these homsets and the diagonal maps. We shall apply this reasoning below to specialise our categorical change of base result which is the subject of the next section.

\subsection{Categorical change of base}

The work below is going to rely on categorical change of base, [T04]. Since the main lemma relevant to this can be stated under very mild categorical assumptions and is easy to prove, it seems appropriate to start with this aspect. We first assume an order enriched cartesian category $\mathcal{C}$ with some distinguished object $\mathbb{S}$.

Let $X$ be an object of $\mathcal{C}$. We use $\mathbb{S}_{X}$ to denote $\pi_{2}: \mathbb{S} \times X \rightarrow X$, an object of the slice $\mathcal{C} / X$. Then,

Lemma 2.2.1. For any morphism $f: X \rightarrow Y$ of $\mathcal{C}$, define

$$
f^{\#}:\left[(\mathcal{C} / Y)^{o p}, \text { Set }\right] \rightarrow\left[(\mathcal{C} / X)^{o p}, \text { Set }\right]
$$

by precomposition with $\Sigma_{f}$ and define

$$
f_{*}:\left[(\mathcal{C} / X)^{o p}, \mathbf{S e t}\right] \rightarrow\left[(\mathcal{C} / Y)^{o p}, \text { Set }\right]
$$

by precomposition with $f^{*}$. Then $f^{\#} \dashv f_{*}$ and for any objects $A, B$ of $\mathcal{C} / X$, $\mathcal{C} / Y$ respectively we have

$$
f^{\#} \mathbb{S}_{Y}^{B} \cong \mathbb{S}_{X}^{f^{*} B}
$$

and

$$
f_{*} \mathbb{S}_{X}^{A} \cong \mathbb{S}_{Y}^{\Sigma_{f} A}
$$

Further there is a natural order isomorphism between the sets of natural transformations,

$$
\left[\mathbb{S}_{X}^{f^{*} B}, \mathbb{S}_{X}^{A}\right] \cong\left[\mathbb{S}_{Y}^{B}, \mathbb{S}_{Y}^{\Sigma_{f} A}\right]
$$

ordered pointwise in the obvious manner.

Here standard notation for the pullback adjunction $\Sigma_{f} \dashv f^{*}: \mathcal{C} / X \rightarrow \mathcal{C} / Y$ is being used.

Proof. To see that $f^{\#} \dashv f_{*}$ observe that the unit and counit of $\Sigma_{f} \dashv f^{*}$ can be used to define natural transformations $f^{\#} f_{*} F \longrightarrow F$ for each $F:(\mathcal{C} / X)^{o p} \rightarrow$ Set and $f_{*} f^{\#} G \longrightarrow G$ for each $G:(\mathcal{C} / Y)^{o p} \rightarrow$ Set. But the induced natural 
transformations $f^{\#} f_{*} \rightarrow I d$ and $f_{*} f^{\#} \rightarrow I d$ can be seen to satisfy the triangular identities for $f^{\#}$ and $f_{*}$ (since the counit and unit of $\Sigma_{f} \dashv f^{*}$ satisfy the triangular identities for $f^{*}$ and $\Sigma_{f}$ ) and so $f^{\#} \dashv f_{*}$.

Both $f^{\#} \mathbb{S}_{Y}^{B} \cong \mathbb{S}_{X}^{f^{*} B}$ and $f_{*} \mathbb{S}_{X}^{A} \cong \mathbb{S}_{Y}^{\Sigma_{f} A}$ are immediate since

$$
\mathcal{C} / Y\left(B \times \Sigma_{f} A, \mathbb{S}_{Y}\right) \cong \mathcal{C} / X\left(f^{*} B \times A, \mathbb{S}_{X}\right)
$$

holds for every $A$ in $\mathcal{C} / X$ and every $B$ in $\mathcal{C} / Y$.

The following lemma will be used in our main technical result on the slice stability of Axiom 4 (to follow). It is essentially an exercise in the definition of $f^{\#}$ and $f_{*}$ just given.

Lemma 2.2.2. For any $W \stackrel{l}{\rightarrow} Y$, a morphism of an order enriched cartesian category $\mathcal{C}$ with some distinguished object $\mathbb{S}$, and for any natural transformation $\alpha: \mathbb{S}_{Y}^{A} \rightarrow \mathbb{S}_{Y}^{B}$ with objects $A$ and $B$ of $\mathcal{C} / Y$ and any object $X$ of $\mathcal{C}$ we have that

$$
\left[W_{*} l^{\#}(\alpha)\right]_{X}=\alpha_{\Sigma_{l} W^{*} X}
$$

and

$$
\alpha_{W_{l}}=\left[W_{*} l^{\#}(\alpha)\right]_{1} .
$$

Note we are using $W_{l}$ as notation for the object $l: W \rightarrow Y$ of $\mathcal{C} / Y$. Further note that we are, of course, using the notation $\Sigma_{W}$ and $W^{*}$ for the pullback adjunction $\mathcal{C} / W \rightleftarrows \mathcal{C}$ and are using $W^{\#} \dashv W_{*}$ (rather than $\left(!^{W}\right)^{\#} \dashv !_{*}^{W}$ ) for the extension to natural transformations.

Proof. In summary the proof is about unwinding the definition of the extensions of the functors $\Sigma_{W}$ and $l^{*}$ to natural transformations.

Firstly consider some natural transformation $\gamma: \mathbb{S}_{W}^{C} \rightarrow \mathbb{S}_{W}^{D}$, then $\left[W_{*}(\gamma)\right]_{X}$ for any object $X$ of $\mathcal{C}$ is defined by

$$
\begin{aligned}
\mathcal{C}\left(X \times \Sigma_{W}(C), \mathbb{S}\right) & \cong \mathcal{C} / W\left(W^{*} X \times{ }_{W} C, \mathbb{S}_{W}\right) \\
\stackrel{\gamma_{W^{*} X}}{\longrightarrow} \mathcal{C} / W\left(W^{*} X \times_{W} D, \mathbb{S}_{W}\right) & \cong \mathcal{C}\left(X \times \Sigma_{W}(D), \mathbb{S}\right) .
\end{aligned}
$$

I.e. $\left[W_{*}(\gamma)\right]_{X}=\gamma_{W^{*} X}$. by

Secondly, for $\alpha: \mathbb{S}_{Y}^{A} \rightarrow \mathbb{S}_{Y}^{B}$ and for any object $E$ of $\mathcal{C} / W,\left[l^{\#}(\alpha)\right]_{E}$ is defined

$$
\begin{aligned}
\mathcal{C} / W\left(E \times_{W} l^{*} A, \mathbb{S}_{W}\right) & \cong \mathcal{C} / Y\left(\Sigma_{l} E \times_{Y} A, \mathbb{S}_{Y}\right) \\
\stackrel{\alpha_{\Sigma_{l} E}}{\longrightarrow} \mathcal{C} / Y\left(\Sigma_{l} E \times_{Y} B, \mathbb{S}_{Y}\right) & \cong \mathcal{C} / W\left(E \times_{W} l^{*} B, \mathbb{S}_{W}\right)
\end{aligned}
$$

I.e. $\left[l^{\#}(\alpha)\right]_{E}=\alpha_{\Sigma_{l} E}$. Combining these we get the first claim. For the second claim note that $\Sigma_{l} W^{*} 1=W_{l}$.

We now discuss a sharpening of Lemma 2.2.1 for situations where the additional assumption is made that $\mathbb{S}$ is an order internal join semilattice. Certainly then $\mathbb{S}_{Y}^{\Sigma_{f}(-)}$ transforms $\mathbb{S}_{X}^{A}$ into an order internal join semilattice in $\left[\mathcal{C} / Y^{o p}\right.$, Set $]$ 
as the lemma shows it is (a restriction of) a right adjoint. We would like to conclude, moving in the opposite direction, that $\mathbb{S}_{X}^{f^{*}\left({ }^{-}\right)}$transforms any $\mathbb{S}_{Y}^{B}$ into a join semilattice in $\left[\mathcal{C} / X^{o p}\right.$, Set]. If $h^{*}: \mathcal{C} / W \rightarrow \mathcal{C} / Z$ preserves finite coproduct (for every morphism $h: Z \rightarrow W$ ) then this is the case since then $\mathbb{S}_{Y}^{B} \times \mathbb{S}_{Y}^{B} \cong \mathbb{S}_{Y}^{B+B}$ and $\mathbb{S}_{X}^{f^{*}(B+B)} \cong \mathbb{S}_{X}^{f^{*} B+f^{*} B} \cong \mathbb{S}_{Y}^{f^{*} B} \times \mathbb{S}_{Y}^{f^{*} B}$, showing that $\mathbb{S}_{X}^{f^{*}(-)}$ preserves binary product (nullary product is similar). In summary,

Proposition 2.2.3. If $\mathcal{C}$ is a cartesian order enriched category with finite coproducts such that pullback preserves finite coproducts then for any order internal join semilattice $\mathbb{S}$, any $f: X \rightarrow Y$ and any $A$ and $B$ objects of $\mathcal{C} / X$ and $\mathcal{C} / Y$ respectively,

(i) $\mathbb{S}_{Y}^{\Sigma_{f}(-)}$ takes $\sqcup_{\mathbb{S}_{X}^{A}}$ to $\sqcup_{\mathbb{S}_{Y}^{\Sigma_{f}}}$ (and similarly nullary join),

(ii) $\mathbb{S}_{X}^{f^{*}\left({ }_{-}\right)}$takes $\sqcup_{\mathbb{S}_{Y}^{B}}$ to $\sqcup_{\mathbb{S}_{X}^{f^{*} B}}$ (and similarly nullary join), and;

(iii) the order isomorphism between natural transformations of Lemma 2.2.1 specialises to natural transformations that are join semilattice homomorphisms.

Proof. (i) and (ii) are covered in the preamble. For (iii) note that the unit and counit of the adjunction in the lemma are

$$
\mathbb{S}_{Y}^{B} \stackrel{\mathbb{S}_{Z}^{\epsilon_{B}}}{\rightarrow} \mathbb{S}_{Y}^{\Sigma_{f} f^{*} B}
$$

and

$$
\mathbb{S}_{X}^{f^{*} \Sigma_{f} A} \stackrel{\mathbb{S}_{X}^{\eta}}{\longrightarrow} \mathbb{S}_{X}^{A}
$$

respectively, where $\eta$ and $\epsilon$ are the unit and counit of the adjunction $\Sigma_{f} \dashv f^{*}$. But both these maps are internal join semilattice homomorphisms and so (iii) is clear as (i) and (ii) show that $\mathbb{S}_{X}^{f^{*}(-)}$ and $\mathbb{S}_{Y}^{\Sigma_{f}(-)}$, on morphisms, preserve the property of being an internal join semilattice homomorphism.

Of course an identical discussion could have been had about order internal meet semilattices.

\section{The axioms}

\subsection{Basic axioms}

We now state various axioms that can be placed on a category $\mathcal{C}$ and show that they are slice stable. They are all stable under the order enrichment which means that the theory developed has an implicit order enriched duality. The axioms are all true when $\mathcal{C}=$ Loc and are used in [T04] and [T05] to develop various aspects of locale theory.

Axiom 1. $\mathcal{C}$ is an order enriched category with finite limits and finite colimits.

It has been commented on above that the property of being cartesian is slice stable. It is similarly trivial that the property of being cocartesian is slice stable. 
Axiom 2. For any objects $A, B$ and $C$ in $\mathcal{C} / Z, A \times(B+C) \cong A \times B+A \times C$. Further $A \times 0 \cong 0$.

This axiom is about all slices and so is, by definition, a slice stable axiom. It is equivalent to the assertion that for any morphism $f: X \rightarrow Y$ of $\mathcal{C}$, the pullback functor $f^{*}: \mathcal{C} / X \rightarrow \mathcal{C} / Y$ preserves finite coproducts. (I have not been able to exhibit a category that is distributive for which this axiom fails. Although it is surely know whether distributivity is sufficient for Axiom 2, it has not proved possible here to establish this either way.)

Axiom 3. There is an order internal distributive lattice, denoted $\mathbb{S}$, such that given a pullback

$$
\begin{array}{ccc}
a^{*}(i) & \rightarrow & 1 \\
\downarrow & & \downarrow i \\
X & \stackrel{a}{\rightarrow} & \mathbb{S}
\end{array}
$$

$a$ is uniquely determined by $a^{*}(i) \hookrightarrow X$ for $i: 1 \rightarrow \mathbb{S}$ equal to either $0_{\mathbb{S}}$ or $1_{\mathbb{S}}$.

Such an $\mathbb{S}$ is called a Sierpinski object. If $\mathbb{S}$ is a Sierpiński object in $\mathcal{C}$ then for any object $Z$ of $\mathcal{C}$ it is easy to verify that $\mathbb{S}_{Z}$ is a Sierpiński object of $\mathcal{C} / Z$ so the axiom is slice stable. It can be shown, [T04], that this axiom implies, for any object $Z$ of $\mathcal{C}$ and any morphism $\alpha: \mathbb{S}^{Z} \rightarrow \mathbb{S}$,

$$
\text { (i) } \Pi_{\mathbb{S}}\left(\alpha \times I d_{\mathbb{S}}\right) \sqsubseteq \alpha \Pi_{\mathbb{S} Z}\left(I d_{\mathbb{S} z} \times \mathbb{S}^{!^{z}}\right) \text {; and }
$$

(ii) $\alpha \sqcup_{\mathbb{S} z}\left(I d_{\mathbb{S} Z} \times \mathbb{S}^{!^{Z}}\right) \sqsubseteq \sqcup_{\mathbb{S}}\left(\alpha \times I d_{\mathbb{S}}\right)$.

It is these last two inequalities which are sufficient to develop certain aspects of locale theory and so are taken as axioms in [T05] rather than our Axiom 3.

Capturing the usual localic notion we have:

Definition 3.1.1. A monomorphism $X_{0} \hookrightarrow X$ is an open subobject if it is the pullback of $1_{\mathbb{S}}: 1 \rightarrow \mathbb{S}$ and it is a closed subobject if it is the pullback of $0_{\mathbb{S}}: 1 \rightarrow \mathbb{S}$.

It is shown in [T04] that this axiom establishes an order isomorphism between open subobjects of $X$ and morphisms $X \rightarrow \mathbb{S}$; in particular to prove that $a \sqsubseteq b$ for $a, b: X \rightarrow \mathbb{S}$ it is sufficient to prove that for any morphism $Z \stackrel{p}{\rightarrow} X$ if $p$ factors via $a^{*} 1$ then $p$ factors via $b^{*} 1$.

Although in locale theory the Sierpiński locale can be defined categorically (for example as the initial order internal distributive lattice, or as the categorical tensor $1 \otimes \mathbf{2}$ where $\mathbf{2}$ is the external poset $\{0 \leq 1\}$ ) it is not the case that Axiom 3 determines an object unique up to isomorphism. To see this note that the terminal object always satisfies Axiom 3. However this lack of uniqueness makes no difference to the development of the theory to follow.

The next axiom is a categorical interpretation of the content of the double coverage theorem introduced in [VT04].

Axiom 4. For any equalizer diagram

$$
E \stackrel{e}{\hookrightarrow} X \underset{g}{\stackrel{f}{\rightarrow}} Y
$$


in $\mathcal{C}$ the diagram

$$
\mathbb{S}^{X} \times \mathbb{S}^{X} \times \mathbb{S}^{Y} \stackrel{\sqcap(1 \times \sqcup)\left(I d \times I d \times \mathbb{S}^{f}\right)}{\stackrel{\longrightarrow}{\longrightarrow}} \mathbb{S}^{X} \stackrel{\mathbb{S}^{e}}{\rightarrow} \mathbb{S}^{E}
$$

is a coequalizer in the full subcategory of $\left[\mathcal{C}^{o p}\right.$, Set $]$ consisting of all objects of the form $\mathbb{S}^{W}$.

Here $\sqcap(1 \times \sqcup)$ is the composite

$$
\mathbb{S}^{X} \times \mathbb{S}^{X} \times \mathbb{S}^{X} \stackrel{1 \times \amalg}{\longrightarrow} \mathbb{S}^{X} \times \mathbb{S}^{X} \stackrel{\sqcap}{\longrightarrow} \mathbb{S}^{X} .
$$

Our main technical result for this paper is a proof that this axiom is slice stable.

Proposition 3.1.2. Assuming Axioms 1-3, Axiom 4 is slice stable.

Proof. Say $E \stackrel{e}{\stackrel{e}{\hookrightarrow}} X_{1} \underset{h_{2}}{\stackrel{h_{1}}{\rightarrow}} X_{2}$ is an equalizer diagram in $\mathcal{C} / Y$, we must show that for any $\alpha: \mathbb{S}_{Y}^{X_{1}} \rightarrow \mathbb{S}_{Y}^{Z}$ for which

$$
\begin{aligned}
& \alpha \sqcap(1 \times \sqcup)\left(I d \times I d \times \mathbb{S}_{Y}^{h_{1}}\right) \\
= & \alpha \sqcap(1 \times \sqcup)\left(I d \times I d \times \mathbb{S}_{Y}^{h_{2}}\right) \quad \text { Eqn } 1 .
\end{aligned}
$$

there exists unique $\beta: \mathbb{S}_{Y}^{E} \rightarrow \mathbb{S}_{Y}^{Z}$ such that $\beta \mathbb{S}_{Y}^{e}=\alpha$. The first thing to note is that by application of the change of base proposition above we can assume that $Z=1$ (recall that change of base preserves Sierpiński meet and join; Proposition 2.2.3).

So say we are given $\alpha: \mathbb{S}_{Y}^{X_{1}} \rightarrow \mathbb{S}_{Y}$ satisfying Eqn 1 . To define $\beta: \mathbb{S}_{Y}^{E} \rightarrow \mathbb{S}_{Y}$ we must, for every $l: W \rightarrow Y$, define a map

$$
\mathcal{C} / Y\left(E \times_{Y} W_{l}, \mathbb{S}_{Y}\right) \rightarrow \mathcal{C} / Y\left(W_{l}, \mathbb{S}_{Y}\right) .
$$

Since $\mathcal{C} / Y\left(E \times_{Y} W_{l}, \mathbb{S}_{Y}\right) \cong \mathcal{C}\left(E \times_{Y} W, \mathbb{S}\right)$ and $\mathcal{C} / Y\left(W_{l}, \mathbb{S}_{Y}\right) \cong \mathcal{C}(W, \mathbb{S})$ this amounts to defining a map

$$
\mathcal{C}\left(E \times_{Y} W, \mathbb{S}\right) \rightarrow \mathcal{C}(W, \mathbb{S})
$$

for each $l: W \rightarrow Y$. Now if $\alpha$ satisfies Eqn 1 then $W_{*} l \#(\alpha)$ satisfies

$$
\begin{aligned}
& \left(W_{*} l^{\#}(\alpha)\right) \sqcap(1 \times \sqcup)\left(I d \times I d \times \mathbb{S}^{h_{1} \times I d}\right) \\
= & \left(W_{*} l^{\#}\right) \sqcap(1 \times \sqcup)\left(I d \times I d \times \mathbb{S}^{h_{2} \times I d}\right) .
\end{aligned}
$$

since, again by change of base, the extended functors $W_{*}$ and $l^{\#}$ preserve the Sierpiński meet and join. Therefore, by Axiom 4, there exists a unique natural

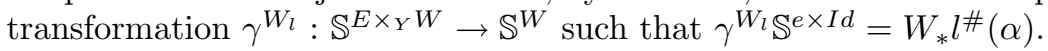

We define $\beta: \mathbb{S}_{Y}^{E} \rightarrow \mathbb{S}_{Y}$ by $\beta_{W_{l}} \equiv\left[\gamma^{W_{l}}\right]_{1}$. The construction of $\beta$ from $\alpha$ is monotone so to complete the proof it remains to verify,

A. that $\beta$ is natural, 
B. $\beta \mathbb{S}_{Y}^{e}=\alpha$; and,

C. if $\delta \mathbb{S}_{Y}^{e}=\alpha$ for some other natural transformation $\delta: \mathbb{S}_{Y}^{E} \rightarrow \mathbb{S}_{Y}$ then $\delta=\beta$.

Proof of $A$. Say $n: W_{l} \rightarrow V_{m}$ is a morphism in $\mathcal{C} / Y$ then by the first part of the lemma the square

$$
\begin{array}{ccc}
\mathbb{S}^{V \times{ }_{Y} X_{1}} & \Sigma_{V} n^{*}(\alpha) & \mathbb{S}^{V} \\
\mathbb{S}^{n \times I d} \downarrow & & \downarrow \mathbb{S}^{n} \\
\mathbb{S}^{W \times{ }_{Y} X_{1}} & \stackrel{\Sigma_{W} l^{*}(\alpha)}{\longrightarrow} & \mathbb{S}^{W}
\end{array}
$$

commutes by naturality of $\alpha$. However $\Sigma_{V} n^{*}(\alpha)$ factors as $\gamma^{V_{n}} \mathbb{S}^{e \times I d}$ and $\Sigma_{W} l^{*}(\alpha)$ factors as $\gamma^{W_{l}} \mathbb{S}^{e \times I d}$ and since $\mathbb{S}^{e \times I d}$ is an epimorphism we can conclude that

$$
\begin{array}{lll}
\mathbb{S}^{V \times_{Y} E} & \stackrel{\gamma^{V_{n}}}{\longrightarrow} & \mathbb{S}^{V} \\
\mathbb{S}^{n \times I d} \downarrow & & \downarrow \mathbb{S}^{n} \\
\mathbb{S}^{W \times_{Y} E} & \stackrel{\gamma^{W_{l}}}{\longrightarrow} & \mathbb{S}^{W}
\end{array}
$$

commutes. By applying these natural transformations at 1 we therefore obtain the fact that $\beta$ is natural.

Proof of B. By the lemma, for any $W_{l}, \alpha_{W_{l}}=\left[\Sigma_{W} l^{*}(\alpha)\right]_{1}=\left[\gamma^{W_{l}} \mathbb{S}^{e \times I d}\right]_{1}=$ $\left[\gamma^{W_{l}}\right]_{1}\left[\mathbb{S}^{e \times I d}\right]_{1}=\beta_{W_{l}}\left[\mathbb{S}_{Y}^{e}\right]_{W_{l}}$.

Proof of $C$. For such a $\delta$, for any $W_{l}, \Sigma_{W} l^{*}(\alpha)=\Sigma_{W} l^{*}(\delta) \mathbb{S}^{e \times I d}$, by applying $\Sigma_{W} l^{*}$ to the triangle $\delta \mathbb{S}_{Y}^{e}=\alpha$. It follows that $\Sigma_{W} l^{*}(\delta)=\gamma^{W_{l}}$ by the uniqueness part of Axiom 4. But by the lemma $\delta_{W_{l}}=\left[\Sigma_{W} l^{*}(\delta)\right]_{1}=\left[\gamma^{W_{l}}\right]_{1}=\beta_{W_{l}}$ and so we are done.

Axiom 5. The map $\mathbb{S}^{(-)}: \mathcal{C} \rightarrow\left[\mathcal{C}^{o p}\right.$, Set $]$, reflects isomorphisms.

Given Axiom 4, Axiom 5 implies that $\mathbb{S}^{(-)}$is faithful. To see this note that if $g_{1}$ and $g_{2}$ are two morphisms with $\mathbb{S}^{g_{1}}=\mathbb{S}^{g_{2}}$ then $\mathbb{S}^{e}$ is an isomorphism by Axiom 4, where $e$ is the equalizer of $g_{1}$ and $g_{2}$. This is sufficient to show that $g_{1}=g_{2}$ as $e$ is therefore an isomorphism in the presence of Axiom 5 .

For slice stability of Axiom 5, say $\mathbb{S}_{Y}^{f}: \mathbb{S}_{Y}^{W_{l}} \rightarrow \mathbb{S}_{Y}^{V_{m}}$ is an isomorphism in $\left[(\mathcal{C} / Y)^{o p}\right.$, Set $]$, for some morphism $f$ of $\mathcal{C} / Y$. Then $\Sigma_{Y}\left(\mathbb{S}_{Y}^{f}\right)$, i.e. $\mathbb{S}^{f}: \mathbb{S}^{W} \rightarrow \mathbb{S}^{V}$, is an isomorphism in $\left[\mathcal{C}^{o p}\right.$, Set $]$ and so $f$ is an isomorphism in $\mathcal{C}$ by the axiom. Therefore there exists $g: W \rightarrow V$ such that $g f=I d_{V}$ and $g f=I d_{W}$. It must just be checked that $g$ is a morphism of $\mathcal{C} / Y$, i.e. that $m g=l$. As $f$ is a morphism of $\mathcal{C} / Y$ we gave $l f=m$. Therefore $m g=l f g=l$ and we are done.

In [T05] a (not necessarily strict) strengthening of Axiom 5 is exploited so for completeness this is included here.

Axiom 5'. Any internal distributive lattice homomorphism $\alpha: \mathbb{S}^{Y} \rightarrow \mathbb{S}^{X}$ is of the form $\mathbb{S}^{f}$ for unique $f: X \rightarrow Y$.

Proposition 3.1.3. Given Axioms 1-4, Axiom $5^{\prime}$ is slice stable.

Proof. Say $\alpha: \mathbb{S}_{Y}^{W_{l}} \rightarrow \mathbb{S}_{Y}^{V_{m}}$ is an internal distributive lattice homomorphism. By change base to $V$, we obtain $\widehat{\alpha}: \mathbb{S}_{V}^{m^{*} W_{l}} \rightarrow \mathbb{S}_{V}$, the adjoint transpose of $\alpha$, 
which is also an internal distributive lattice homomorphism. Now by Axiom 3 applied to $\mathcal{C} / Y$, the relations

$$
\begin{gathered}
\text { (i) } \sqcap(\widehat{\alpha} \times 1) \sqsubseteq \widehat{\alpha} \sqcap\left(1 \times \mathbb{S}_{V}^{m^{m^{*} W_{l}}}\right) \text {; and } \\
\text { (ii) } \widehat{\alpha} \sqcup\left(1 \times \mathbb{S}_{V}^{m^{m^{*} W_{l}}}\right) \sqsubseteq \sqcup(\widehat{\alpha} \times 1)
\end{gathered}
$$

hold and since $\widehat{\alpha}$ preserves 0 and 1 , this shows that $\widehat{\alpha}$ is split by $\mathbb{S}_{V}^{!}$; in other words the diagram

$$
\begin{array}{lll}
\mathbb{S}_{V}^{m^{*} W_{l}} & \stackrel{\widehat{\alpha}}{\longrightarrow} & \mathbb{S}_{V} \\
& \mathbb{S}_{V}^{m^{m^{*} W_{l}}} \nwarrow & \begin{array}{c}
\uparrow I d \\
\mathbb{S}_{V}
\end{array}
\end{array}
$$

commutes. By taking adjoint transpose (i.e. changing base) back to $Y$, we get that

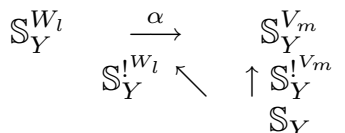

commutes. Then by applying the functor $\Sigma_{Y}$ to this triangle we see both that $\Sigma_{Y}(\alpha)$, since it is a distributive lattice homomorphism, is $\mathbb{S}^{f}$ for some $f: V \rightarrow$ $W$ and also, by the uniqueness part of Axiom $5^{\prime}$, that $f m=l$. This shows that $f$ is a morphism of $\mathcal{C} / Y$. To prove that $\alpha=\mathbb{S}_{Y}^{f}$ it is sufficient to check that

$$
\alpha \mathbb{S}_{Y}^{(I d, f)}=\mathbb{S}_{Y}^{f} \mathbb{S}_{Y}^{(I d, f)}
$$

since $(I d, f)$ is an regular monomorphism (apply Axiom 4). But (*) follows since the adjoint transpose (back to 1 ) of both sides is $\Sigma_{Y}(\alpha)$.

Axiom 6: For any regular epimorphism $q: X \rightarrow Q$ in $\mathcal{C}$, any for any $\alpha$, $\beta: \mathbb{S}^{Y} \rightarrow \mathbb{S}^{Q}$, if $\mathbb{S}^{q} \alpha=\mathbb{S}^{q} \beta$ then $\alpha=\beta$.

In other words, if $q$ is a regular epimorphism then $\mathbb{S}^{q}$ is a monomorphism in the full subcategory of $\left[\mathcal{C}^{o p}\right.$, Set] consisting of all objects of the form $\mathbb{S}^{Y}$. Note that in [T04] this aspect of the axiomatisation is presented as

"The image of a coequalizer diagram under $\mathbb{S}^{(-)}: \mathcal{C} \rightarrow\left[\mathcal{C}^{o p}\right.$, Set $]$ is an equalizer diagram provided we restrict to the full category of all objects in $\left[\mathcal{C}^{o p}, \mathbf{S e t}\right]$ of the form $\mathbb{S}^{X}$ ".

However, in application it is only the fact that $\mathbb{S}^{q}$ is a monomorphism that is used, so all the results of [T04] are still available with the weakening just given.

Proposition 3.1.4. Assuming Axioms 1-4, Axiom 6 is slice stable.

Proof. Say $X \stackrel{q}{\longrightarrow} Q$ is a regular epimorphism in $\mathcal{C} / Y$ and that we are given $\alpha$, $\beta: \mathbb{S}_{Y}^{W_{l}} \rightarrow \mathbb{S}_{Y}^{Q}$ with $\mathbb{S}_{Y}^{q} \alpha=\mathbb{S}_{Y}^{q} \beta$ for any object $W_{l}$ of $\mathcal{C} / Y$. Since $W_{l}$ exists as an equalizer

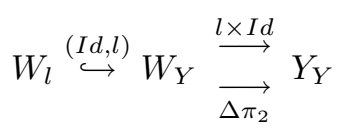


in $\mathcal{C} / Y$, to prove that $\alpha=\beta$ it is sufficient to prove that $\alpha \mathbb{S}_{Y}^{(I d, l)}=\beta \mathbb{S}_{Y}^{(I d, l)}$ and appeal to Axiom 4. But the adjoint transpose of $\alpha \mathbb{S}_{Y}^{(I d, l)}$ under change of base back to 1 is $\Sigma_{Y}(\alpha)$ and, identically, the adjoint transpose of $\beta \mathbb{S}_{Y}^{(I d, l)}$ is $\Sigma_{Y}(\beta)$; so it remains to prove $\Sigma_{Y}(\alpha)=\Sigma_{Y}(\beta)$ which is trivial by application of Axiom 6 to $q$ as $q$ is certainly a regular epimorphism in $\mathcal{C}$ (as coequalizers in $\mathcal{C} / Y$ are created in $\mathcal{C}$ ).

In summary we have therefore now shown that all of the axiomatic account of weak triquotient assignments in locale theory, which is the main topic of [T04], is automatically slice stable. The slice stable phrasing of the axioms in that paper is redundant.

\subsection{The double power object axiom}

To complete this discussion it is useful to point out that various other categorical axioms for locales are also automatically slice stable. The most important is probably the next axiom, which offers a categorical characterization of the double power object:

Axiom 7. The functor $\mathbb{S}^{(-)}: \mathcal{C} \rightarrow\left[\mathcal{C}^{o p}\right.$, Set $]$, once its codomain is restricted to the full category of all objects in $\left[\mathcal{C}^{o p}\right.$, Set $]$ of the form $\mathbb{S}^{X}$, has a right adjoint.

Such a right adjoint, it can be verified, sends $\mathbb{S}^{X}$ to $\mathbb{S}^{\mathbb{S}^{X}}$, i.e. the double exponential. Conversely if such an exponential exists and is representable then the axiom is satisfied. The notation $\mathbb{P}(X)=\mathbb{S}^{\mathbb{S}^{X}}$ is used and it can be verified that a monad is defined on $\mathcal{C}$ using the universal properties of exponentiation. With this axiom we can stop writing 'the full subcategory of $\left[\mathcal{C}^{o p}\right.$, Set $]$ consisting of all objects of the form $\mathbb{S}^{X}$, and write $\mathcal{C}_{\mathbb{P}}^{o p}$, since this full subcategory is readily seen to be (weakly) equivalent to the opposite of the Kleisli category. Note that Axiom 4 implies that $\mathbb{P}$ takes regular monomorphisms to regular monomorphisms. This is not to say that it preserves equalizer diagrams, though it can be checked that it preserves coreflexive equalizers.

It is worth noting further that this axiom is a (not necessarily strict) strengthening of Axiom 6.

Proposition 3.2.1. Given Axioms 1-4, Axiom 7 is slice stable.

Proof. Firstly notice that for any objects $X$ and $Y$ of $\mathcal{C}$, the double exponential $\mathbb{S}_{Y}^{\mathbb{S}_{Y}^{X_{Y}}}$ exists in $\left[(\mathcal{C} / Y)^{o p}\right.$, Set $]$. It is given by $\mathbb{P}(X)_{Y}$. This can be verified by change of base since, for any object $W_{l}$ of $\mathcal{C} / Y$,

$$
\begin{aligned}
\mathcal{C} / Y\left(W_{l}, \mathbb{P}(X)_{Y}\right) & \cong \mathcal{C}(W, \mathbb{P}(X)) \\
& \cong \operatorname{Nat}\left[\mathbb{S}^{X}, \mathbb{S}^{W}\right] \\
& \cong \operatorname{Nat}\left[\mathbb{S}_{Y}^{X_{Y}}, \mathbb{S}_{Y}^{W_{l}}\right]
\end{aligned}
$$

where the last line is by change of base. 
Now, as in the previous proof any $X_{f}$, an object of $\mathcal{C} / Y$, occurs as an equalizer

$$
X_{f} \stackrel{(I d, f)}{\hookrightarrow} X_{Y} \stackrel{f \times I d}{\stackrel{f \times}{\longrightarrow}} Y_{Y}
$$

and this gives rise, via Axiom 4 in the slice $\mathcal{C} / Y$, to a coequalizer in (a full subcategory of $)\left[(\mathcal{C} / Y)^{o p}\right.$, Set $]$ which we can write as

$$
\mathbb{S}_{Y}^{(X+X+Y)_{Y}} \stackrel{\stackrel{\alpha}{\longrightarrow}}{\longrightarrow} \mathbb{S}_{Y}^{X_{Y}} \stackrel{\mathbb{S}_{Y}^{(I d, f)}}{\longrightarrow} \mathbb{S}_{Y}^{X_{f}}
$$

If we therefore define $\mathbb{P}_{Y}\left(X_{f}\right)$ to be the equalizer of

$$
\mathbb{P}(X)_{Y} \underset{\mathbb{S}_{Y}^{\beta}}{\stackrel{\mathbb{S}_{Y}^{\alpha}}{\longrightarrow}} \mathbb{P}(X+X+Y)_{Y}
$$

it clearly then has the right universal property of the double exponential.

\subsection{The upper and lower power monad axiom}

In [T05] an axiomatic account of the upper and lower power constructions is developed. Both are submonads of the double power monad. The defining characteristic of these power constructions, denoted $P_{U}$ and $P_{L}$ respectively, is that their points (i.e. morphism $\mathcal{C}\left(Z, P_{U}(X)\right), \mathcal{C}\left(Z, P_{L}(X)\right)$ respectively) are in order isomorphism with internal meet (respectively join) semilattice homomorphisms $\mathbb{S}^{X} \rightarrow \mathbb{S}^{Z}$. Notice that just as in the double power construction it is easy, by change of base, to check that $P_{U}^{Y}\left(X_{Y}\right) \cong P_{U}(X)_{Y}$ and $P_{L}^{Y}\left(X_{Y}\right) \cong P_{L}(X)_{Y}$ where the $Y$ in $P_{L}^{Y}$ indicates the lower power construction relative to $\mathcal{C} / Y$, and similarly for the upper power construction. Explicitly $P_{L}(X) \stackrel{j_{X}^{L}}{\longrightarrow} \mathbb{P}(X)$ is constructed as the equalizer of

$$
\mathbb{P}(X) \underset{\left(\mathbb{S}_{\mathbb{S} X}^{0_{\mathbb{S}}}, g\right)}{\left.\stackrel{\left(0_{\mathbb{S}} \mathbb{P}^{(X)}\right.}{\longrightarrow}, f\right)} \mathbb{S} \times \mathbb{P}(X+X)
$$

where $f$ is the exponential transpose of

$$
\begin{aligned}
\mathbb{S}^{\mathbb{S}^{X}} \times \mathbb{S}^{X} \times \mathbb{S}^{X} & \rightarrow \mathbb{S} \\
(\Lambda, a, b) & \longmapsto \Lambda\left(a \sqcup_{\mathbb{S}^{X}} b\right)
\end{aligned}
$$

and $g$ is the exponential transpose of

$$
\begin{aligned}
\mathbb{S}^{\mathbb{S}^{X}} \times \mathbb{S}^{X} \times \mathbb{S}^{X} & \rightarrow \mathbb{S} \\
(\Lambda, a, b) & \longmapsto \Lambda(a) \sqcup_{\mathbb{S}} \Lambda(b) .
\end{aligned}
$$


The notation $\left(P_{L}, \eta^{L}, \mu^{L}\right)$ is used for the induced monad and we use $\nabla_{X}$ : $\mathbb{S}^{X} \rightarrow \mathbb{S}^{P_{L}(X)}$ for the double exponential transpose of $j_{X}^{L}$. By construction every internal join semilattice homomorphism $\alpha: \mathbb{S}^{X} \rightarrow \mathbb{S}^{Y}$ factors as $\left.\mathbb{S}^{p_{\alpha}}\right\rangle_{X}$ for some unique $p_{\alpha}: Y \rightarrow P_{L}(X)$ and this establishes an order isomorphisms between $\sqcup-S \operatorname{Lat}\left[\mathbb{S}^{X}, \mathbb{S}^{Y}\right]$ and $\mathcal{C}\left(Y, P_{L}(X)\right)$.

To develop a reasonable theory of these power constructions (for example to prove the Hofmann-Mislove theorem) it appears to be necessary to make the additional assumption that $P_{U}$ is co-KZ and, order dually, that $P_{L}$ is $\mathrm{KZ}$. So, given the subject to hand, it makes sense to check that this additional assumption is also slice stable. We will look at the lower construction only; the upper construction is exactly order dual.

Let us first gather a couple of consequences of Axiom 4.

Proposition 3.3.1. A. (lower coverage theorem) If $E \stackrel{e}{\hookrightarrow} X \stackrel{f}{\rightarrow} Y$ is an equalizer diagram in $\mathcal{C}$ then for any join semilattice homomorphism $\alpha: \mathbb{S}^{X} \rightarrow \mathbb{S}^{Z}$ such that

$$
\alpha \sqcap_{\mathbb{S} X}\left(I d \times \mathbb{S}^{f}\right)=\alpha \Pi_{\mathbb{S}^{X}}\left(I d \times \mathbb{S}^{g}\right)
$$

there exists unique join semilattice homomorphism $\beta$ such that $\beta \mathbb{S}^{e}=\alpha$.

$B$. If $e: E \hookrightarrow X$ is a regular monomorphism then so is $P_{L}(e)$.

Proof. A. Given such an $\alpha$, there certainly exists unique $\beta$ such that $\beta \mathbb{S}^{e}=\alpha$; this is Axiom 4. We just need to check that $\beta$ is a join semilattice homomorphism. Certainly $\beta$ preserves 0 since so too do $\mathbb{S}^{e}$ and $\alpha$. To prove that $\beta$ preserves binary join it is sufficient to prove that $\mathbb{S}^{e} \times \mathbb{S}^{e}$ is an epimorphism (in $\left.\mathcal{C}_{\mathbb{P}}\right)$ since both $\mathbb{S}^{e}$ and $\alpha$ preserve binary join. But $\mathbb{S}^{e} \times \mathbb{S}^{e}=\left(\mathbb{S}^{e} \times \mathbb{S}^{1_{E}}\right)\left(\mathbb{S}^{1_{X}} \times \mathbb{S}^{e}\right)$ so it will follow that this morphism is an epimorphism provided we show that $1_{X}+e$ and $e+1_{E}$ are both regular monomorphisms and then apply Axiom 4 twice. But by application of Axiom 2

$$
\begin{array}{ccc}
E+E & \stackrel{[f e, f e]}{\longrightarrow} & Y \\
e+1_{E} \downarrow & & \downarrow \Delta_{Y} \\
X+E & {[(f, g),(f e, f e)]} & Y \times Y
\end{array}
$$

is a pullback diagram and regular monomorphisms (here $\Delta_{Y}$ ) are pullback stable, so $e+1_{E}$ is a regular monomorphism. $1_{X}+e$ is dealt with similarly.

B. This is an application of $\mathrm{A}$ and the defining property of the points of $P_{L}(E)$ and $P_{L}(X)$.

The order dual of Part A of the proposition is stated as an axiom in [T06]. Part B can be applied to show that our final axiom is slice stable.

Axiom 8. The monad $P_{L}$ is $\mathrm{KZ}$ and the monad $P_{U}$ is co-KZ.

Proposition 3.3.2. Given Axioms 1-4 and Axiom 7, Axiom 8 is slice stable. 
Proof. We look at the lower power monad only; the situation with the upper power monad is dual.

To see the proof consider the following diagram constructed from $X_{f}$, an arbitrary object of $\mathcal{C} / Y$ :

$$
\begin{array}{ccccc}
P_{L}^{Y} P_{L}^{Y}\left(X_{f}\right) & \stackrel{\left[\mu_{L}^{Y}\right]_{X_{f}}}{\longrightarrow} & P_{L}^{Y}\left(X_{f}\right) & \stackrel{\left[P_{L}^{Y} \eta_{L}^{Y}\right]_{X_{f}}}{\longrightarrow} & P_{L}^{Y} P_{L}^{Y}\left(X_{f}\right) \\
P_{L}^{Y} P_{L}^{Y}((I d, f)) \downarrow & & P_{L}^{Y}((I d, f)) \downarrow & & P_{L}^{Y} P_{L}^{Y}((I d, f)) \downarrow \\
P_{L}^{Y} P_{L}^{Y}\left(X_{Y}\right) & \stackrel{\left[\mu_{L}^{Y}\right]_{X}}{\longrightarrow} & P_{L}^{Y}\left(X_{Y}\right) & {\left[P_{L}^{Y} \stackrel{\left.\eta_{L}^{Y}\right]_{X_{Y}}}{\longrightarrow}\right.} & P_{L}^{Y} P_{L}^{Y}\left(X_{Y}\right) .
\end{array}
$$

The assertion that the monad defined by $P_{L}$ is KZ is by definition (e.g. Lemma B1.1.12 of [J02]) exactly the assertion that $\mu_{L}$ is right adjoint to $P_{L} \eta_{L}$ in the order enrichment. But since $\mu_{L} \circ P_{L} \eta_{L}=I d$ this is equivalent to the assertion that $P_{L} \eta_{L} \circ \mu_{L} \sqsubseteq I d$. This last assertion is sufficient to show that the bottom row of the diagram is less than or equal to $I d_{P_{L}^{Y} P_{L}^{Y}\left(X_{Y}\right)}$ since we have commented already that $P_{L}^{Y}\left(X_{Y}\right) \cong P_{L}(X)_{Y}$. Therefore $\left[P_{L}^{Y} \eta_{L}^{Y}\right]_{X_{f}} \circ\left[\mu_{L}^{Y}\right]_{X_{f}} \sqsubseteq I d_{P_{L}^{Y}} P_{L}^{Y}\left(X_{f}\right)$ since $P_{L}^{Y} P_{L}^{Y}((I d, f))$ is a regular monomorphism as $(I d, f)$ is.

The axiom given in [T05] relevant to this is the stronger assertion that the Kleisli category $\mathcal{C}_{P_{L}}^{o p}$ is Cauchy complete. It is shown in that paper that an assumption of Cauchy completeness implies that the lower power monad is KZ. In application it is only the $\mathrm{KZ}$ property of the power monad that is exploited and so the result just shown is enough to prove that all the results of [T05] are slice stable.

From now on we shall assume that $\mathcal{C}$ is a category that satisfies all the axioms.

\section{Properties of discrete objects in $\mathcal{C}$}

As an application we now prove some results from locale theory. None of the results are new; the purpose of the exposition is to (i) show that they can all be shown axiomatically and (ii) provide some definitions and lemmas that are necessary for our final section on Joyal and Tierney's localic slice stability result. We only discuss the lower case that arises through our axiomatisation of the lower power monad. The upper case is exactly order dual and is not discussed.

The first subsection recalls the definition of open map and develops some basic results about open maps. We recall how discrete objects can be defined using open maps and that the resulting full subcategory, $\mathbf{D i s}_{\mathcal{C}}$, is regular. The second subsection shows how the order dual of the Hofmann-Mislove theorem can be applied to show that discrete objects are exponentiable. The third subsection describes how the isomorphism inherent in this observation can be defined using relational composition. The final two subsections introduce the ideal completion of a preorder and develop its theory, essentially by applying our axiomatic relational composition to recover what is familiar set theoretically. 
This section is long since the proofs are based on a categorical axiomatisation and so we no longer have certain basic set theoretic results available. The logically more sophisticated reader may wish to simply observe that the usual set theoretic arguments are all available using only the regular fragment of set theory and since the relevant category of discrete objects can be shown axiomatically to be regular all the arguments can be deployed within it. This makes the familiar lattice theoretic proofs available in the axiomatic context that is given and so this provides an alternative route for the proofs in this section. However we have chosen to ignore this approach and just prove the results directly from the axioms.

\subsection{Open maps}

To proceed we are going to need to recall some results about open maps relative to $\mathcal{C}$. Following the usual definition for the case when $\mathcal{C}$ is the category of locales, a morphism $f: X \rightarrow Y$ of $\mathcal{C}$ is said to be open if $\mathbb{S}^{f}$ has a left adjoint $\exists_{f}$ satisfying the Frobenius reciprocity condition

$$
\Pi_{\mathbb{S} Y}\left(\exists_{f} \times I d_{\mathbb{S}^{Y}}\right)=\exists_{f} \Pi_{\mathbb{S} X}\left(I d_{\mathbb{S} X} \times \mathbb{S}^{f}\right) .
$$

The reason for our interest in open maps is that they can be used to determine when an object is discrete:

Definition 4.1.1. An object $X$ of $\mathcal{C}$ is said to be discrete provided the maps $!^{X}: X \rightarrow 1$ and $\Delta_{X}: X \hookrightarrow X \times X$ are open.

This captures the usual localic notion of discrete. We use $\mathbf{D i s}_{\mathcal{C}}$ to denote the fullsubcategory of discrete objects of $\mathcal{C}$.

It can be shown axiomatically that open maps are pullback stable, see [T04]. Standard categorical arguments can be deployed to show that (i) any morphism between discrete objects is open and (ii) finite limits in $\operatorname{Dis}_{\mathcal{C}}$ are created in $\mathcal{C}$.

The paper [T04] also shows that the pullback stability of open maps can be strengthened as follows: if

$$
\begin{array}{ccc}
X \times_{Y} Z & \stackrel{p^{*} f}{\rightarrow} & Z \\
f^{*} p \downarrow & & \downarrow p \\
X & \stackrel{f}{\rightarrow} & Y
\end{array}
$$

is a pullback diagram in $\mathcal{C}$ and $p$ is open, then $\exists_{f^{*} p}$ satisfies the Beck-Chevalley condition; that is $\exists_{f^{*}} \mathbb{S}^{p^{*} f}=\mathbb{S}^{f} \exists_{p}$. Further $\exists_{f^{*} p}$ is unique in the sense that if $\alpha$ : $\mathbb{S}^{X \times_{Y} Z} \rightarrow \mathbb{S}^{X}$ is any other join semilattice homomorphism satisfying Frobenius reciprocity on $f^{*} p$ and $\alpha \mathbb{S}^{p^{*} f}=\mathbb{S}^{f} \exists_{p}$, then $\alpha=\exists_{f^{*} p}$. As an application we show that in certain situations $\exists_{f^{*} p}$ can be calculated explicitly:

Lemma 4.1.2. Given objects $A$ and $Z$ of $\mathcal{C}$, if $!^{A}: A \rightarrow 1$ is open then $\exists_{\pi_{1}}$ : $\mathbb{S}^{Z \times A} \rightarrow \mathbb{S}^{Z}$ is given by

$$
\left[\exists_{\pi_{1}}\right]_{W}(a)=\left[\exists_{A}\right]_{W \times Z}(a)
$$


for each object $W$ of $\mathcal{C}$ and each $a: W \times Z \times A \rightarrow \mathbb{S}$. In other words $\exists_{\pi_{1}}=\exists_{A}^{Z}$ where exponentiation is in $\left[\mathcal{C}^{o p}\right.$, Set $]$.

Of course the notation $\exists_{A}: \mathbb{S}^{A} \rightarrow \mathbb{S}$ is for the left adjoint to $\mathbb{S}^{!^{A}}$. We say that $A$ is open if $!^{A}$ is an open map (though the term overt is also used in the literature). This technical result will be of use later when we are describing the exponentiability of discrete objects.

Proof. Define $\alpha: \mathbb{S}^{Z \times A} \rightarrow \mathbb{S}^{Z}$ by $\alpha_{W}(a)=\left[\exists_{A}\right]_{W \times Z}(a)$. It is routine to verify that this is natural in $W$ and that $\alpha$ is a join semilattice homomorphism since $\exists_{A}$ is. But by naturality of $\exists_{A}$ we have that $\alpha \mathbb{S}^{\pi_{2}}=\mathbb{S}^{!^{Z}} \exists_{A}$ and since $\alpha$ satisfies Frobenius reciprocity on $\pi_{1}: Z \times X \rightarrow Z$ (as $\exists_{A}$ satisfies Frobenius reciprocity on $!^{A}: A \rightarrow 1$ ) we have that $\alpha=\exists_{\pi_{1}}$ by uniqueness of maps satisfying BeckChevalley on the pullback square:

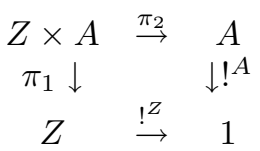

It can also be shown ([T04]) that a subobject is open if and only if it is an open regular monomorphism. In one direction this is shown by observing that the map $\ulcorner\sqcap\urcorner: \mathbb{S} \rightarrow \mathbb{S}^{\mathbb{S}}$, given by the exponential transpose in $\left[\mathcal{C}^{o p}\right.$, Set $]$ of the meet operation $\sqcap: \mathbb{S} \times \mathbb{S} \rightarrow \mathbb{S}$, is a left adjoint to $\mathbb{S}^{\mathbb{S}} \stackrel{\mathbb{S}^{1} \mathbb{S}}{\rightarrow} \mathbb{S}$. This left adjoint, it can be shown, witnesses that $1_{\mathbb{S}}$ is an open map and since $1_{\mathbb{S}}$ is a regular monomorphism (its domain is the terminal object) we have that every open subobject is an open regular monomorphism. In the other direction if $i: X_{0} \hookrightarrow$ $X$ is a regular monomorphism and $i$ is an open map then it can be verified that $i$ is the pullback of $1_{\mathbb{S}}$ along the open $\exists_{i}\left(1_{\mathbb{S} x_{0}}\right)$ (i.e. $\left[\exists_{i}\right]_{1}\left(X_{0} \stackrel{1_{\mathbb{S}} ! X_{0}}{\longrightarrow} \mathbb{S}\right)$ ). It follows that for any open subobject $i: X_{0} \hookrightarrow X$ we have that

$$
\exists_{i} \mathbb{S}^{i}=a \sqcap(-)
$$

where $a=\exists_{i}\left(1_{\mathbb{S} x_{0}}\right)$.

Our final observation about $\operatorname{Dis}_{\mathcal{C}}$, which we again recall from [T04], is that it forms a regular category and so has image factorizations. If $f: X \rightarrow Y$ is a map between discrete objects then it is open and its image is the open subobject $\exists_{f}\left(1_{\mathbb{S} X}\right)$ of $Y$. Note that this also implies that monomorphisms are regular in Dis $_{\mathcal{C}}$ since the image factorization of a monomorphism is itself and open subobjects are regular monomorphisms.

Notation 4.1.3. We need to make a remark on notation. For any natural transformation $\alpha: \mathbb{S}^{X} \rightarrow \mathbb{S}^{Y}$ if $a: 1 \rightarrow \mathbb{S}^{X}$, then of course $\alpha(a): 1 \rightarrow \mathbb{S}^{Y}$ is also by definition a natural transformation. However, by Yoneda's lemma, it is determined uniquely by the open $[\alpha]_{1}\ulcorner a\urcorner$ where $\ulcorner a\urcorner: X \rightarrow \mathbb{S}$ is the exponential transpose of $a$. However in the following we will not use notation to distinguish 
between (i) the morphism $\ulcorner a\urcorner: X \rightarrow \mathbb{S}$, (ii) the natural transformation $a: 1 \rightarrow$ $\mathbb{S}^{X}$ or (iii) the open subobject $(\ulcorner a\urcorner)^{*} 1 \hookrightarrow X$ referring to each ((i), (ii) or (iii)) generically as 'an open of $X$ ' and always using the notation a.

On the other hand for situations where we have $R: X \times Y \rightarrow \mathbb{S}$ we will use $\ulcorner R\urcorner$ for the exponential transpose $X \rightarrow \mathbb{S}^{Y}$. Although this introduces a notational difference between the binary and nullary situations, it does seem to strike the right balance by not over burdening the notation whilst at the same time retaining clarity when multiple variables need to be introduced.

Putting this Remark on notation to use, and as another simple application of the Beck-Chevalley condition on pullback diagrams of open maps, we show that if a discrete object has a point, then the singleton subset corresponding to that point is inhabited:

Lemma 4.1.4. If $A$ is a discrete object of $\mathcal{C}$ and there exists $p: 1 \rightarrow A$ then

$$
\exists_{A}\left(\left\ulcorner\Delta_{A}\right\urcorner p\right)=1_{\mathbb{S}} .
$$

Proof. The open $\left\ulcorner\Delta_{A}\right\urcorner$ p, i.e. $1 \stackrel{p}{\rightarrow} A \stackrel{\left\ulcorner\Delta_{A}\right\urcorner}{\longrightarrow} \mathbb{S}^{A}$, is equal to $A \stackrel{\left(I d_{A}, p !^{A}\right)}{\longrightarrow} A \times A \stackrel{\Delta_{A}}{\longrightarrow} \mathbb{S}$ so following the notational convention just remarked upon,

$$
\begin{aligned}
\left\ulcorner\Delta_{A}\right\urcorner p & =\Delta_{A}\left(I d_{A}, p !^{A}\right) \\
& =\mathbb{S}^{\left(I d_{A}, p !^{A}\right)} \Delta_{A} .
\end{aligned}
$$

By Beck-Chevalley applied to the pullback square

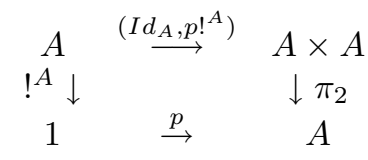

we have that

$$
\begin{aligned}
\exists_{A}\left(\left\ulcorner\Delta_{A}\right\urcorner p\right) & =\exists_{A} \mathbb{S}^{\left(I d_{A}, p^{A}\right)} \Delta_{A} \\
& =\mathbb{S}^{p} \exists_{\pi_{2}} \Delta_{A} \\
& =\mathbb{S}^{p} 1_{\mathbb{S}^{A}} \\
& =1_{\mathbb{S}}
\end{aligned}
$$

where $\exists_{\pi_{2}} \Delta_{A}=1_{\mathbb{S}^{A}}$ since $\Delta_{A}=\exists_{\Delta_{A}}\left(1_{\mathbb{S}^{A}}\right)$ and $\exists_{\pi_{2}} \exists_{\Delta_{A}}=I d_{\mathbb{S}^{A}}$ by uniqueness

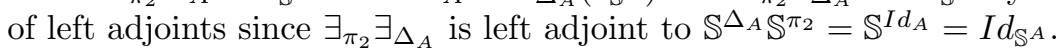

The following lemma will be required below. Intuitively it is showing that any inhabited subset of 1 must be the whole of 1 :

Lemma 4.1.5. If $i: I_{0} \hookrightarrow 1$ is an open subobject of 1 such that $\exists_{i}(1)=1$ then $i$ is an isomorphism. 
Proof. By definition $i$ is the pullback of $1 \stackrel{1_{\mathbb{S}}}{\longrightarrow} \mathbb{S}$ along some $a_{I_{0}}: 1 \rightarrow \mathbb{S}$. BeckChevalley applied to the pullback square implies that $\exists_{i} \mathbb{S}^{I_{0}}=\mathbb{S}^{a_{I_{0}}}\ulcorner\sqcap\urcorner$ where $\ulcorner\sqcap\urcorner$ is the exponential transpose of $\sqcap: \mathbb{S} \times \mathbb{S} \rightarrow \mathbb{S}$. It follows that $\mathbb{S}^{a_{I_{0}}}\ulcorner\sqcap\urcorner(1)=1$ since $\mathbb{S}^{!^{I_{0}}}$ preserves 1 and $\exists_{i}$ preserves 1 by assumption. It follows that $a_{I_{0}}=1_{\mathbf{S}}$ since $\ulcorner\sqcap\urcorner(1)$ is the identity on $\mathbb{S}$ and so $i$ is isomorphic to the identity on 1 .

\subsection{Application of the Hofmann-Mislove theorem to ex- ponentiability}

In this subsection we recall the main result of [T05] which is an axiomatic proof of the existence of an order isomorphism,

$$
\mathcal{C}\left(1, P_{L}(X)\right) \cong\left\{X_{0} \hookrightarrow X \mid X_{0} \text { open, } X_{0} \hookrightarrow X \text { weakly closed }\right\} .
$$

This result can be seen to be the order dual (in the order enrichment of $\mathcal{C}$ ) of the well known Hofmann-Mislove theorem, [HM81]. It is equivalent to BungeFunk's constructive description of the points of the lower power locale, [BF96]. The reason for recalling it is that it, together with a slice stable account of $\mathcal{C}$, allows us to prove axiomatically the well known result (made explicit by Vickers in [V97]) that $\mathbb{S}^{A} \cong P_{L}(A)$ for discrete objects $A$. It is the application of this isomorphism that will guide much of the technical work for the rest of the paper so the final parts of this subsection are devoted to giving an explicit description of how the isomorphism works.

Of course, we must now give the definition of weakly closed:

Definition 4.2.1. A monomorphism $X_{0} \hookrightarrow X$ is a weakly closed subobject if it exists as a lax equalizer of a diagram $f, g: X \rightrightarrows Y$ universally setting $g \sqsubseteq f$ where $f$ factors via the terminal object.

This recovers the usual localic definition in the case where $\mathcal{C}$ is the category of locales.

We must recall how the isomorphism $(*)$ works: it sends a point $p_{\alpha}: 1 \rightarrow$ $P_{L}(X)$ to the lax equalizer universally setting $\eta_{X}^{L}: X \rightarrow P_{L}(X)$ less than or equal to $X \stackrel{!^{X}}{\longrightarrow} 1 \stackrel{p_{\alpha}}{\longrightarrow} P_{L}(X)$. In the other direction, $p_{\exists_{X_{0}} \mathbb{S}^{i}}$ is the point of $P_{L}(X)$ corresponding to weakly closed $X_{0} \stackrel{i}{\hookrightarrow} X$ with $X_{0}$ open.

What happens when $X$ is discrete? We have the following observation which is an axiomatic account of a result in [V97]:

Lemma 4.2.2. If $A$ is a discrete object then for any regular monomorphism $i: X_{0} \hookrightarrow A$ the following are equivalent:

(a) $X_{0}$ is an open object of $\mathcal{C}$ and $i$ is a weakly closed subobject of $A$.

(b) $i$ is an open subobject.

Proof. (a) $\Longrightarrow$ (b). The diagonal $\Delta_{X_{0}}: X_{0} \hookrightarrow X_{0} \times X_{0}$ is open since it is the pullback (along $i \times i$ ) of the open diagonal $\Delta_{A}: A \hookrightarrow A \times A$. Therefore $X_{0}$ is 
discrete and since any morphism between discrete objects is open we have that $i$ is open.

(b) $\Longrightarrow$ (a). Certainly $X_{0}$ is open since the unique map ! ${ }^{X_{0}}$ factors as $X_{0} \stackrel{i}{\hookrightarrow}$ $A \stackrel{!^{A}}{\rightarrow} 1$ and both factors are open. Next we show that $i$ is weakly closed. By definition of being an open subobject, there exists a morphism $a: A \rightarrow \mathbb{S}$ such that $i: a \hookrightarrow A$ is the pullback of $1: 1 \rightarrow \mathbb{S}$ along $a$. We show that $a \hookrightarrow A$ is the lax equalizer universally setting $\eta_{A}^{L}$ less than or equal to $p_{\alpha} !^{A}$ where $\alpha$ is $\mathbb{S}^{A} \stackrel{\mathbb{S}^{i}}{\rightarrow} \mathbb{S}^{a} \stackrel{\exists_{a}}{\longrightarrow} \mathbb{S}$; equivalently $\alpha=\exists_{A}(a \sqcap(-))$. Note firstly that $\eta_{A}^{L} i \sqsubseteq p_{\alpha} !^{A} i$ since $\mathbb{S}^{i} \mathbb{S}^{{ }^{A}}=\mathbb{S}^{!^{X_{0}}}$ and $I d \sqsubseteq \mathbb{S}^{!^{a}} \exists_{a}$ so to complete it is sufficient to check that if there is any $E \stackrel{e}{\rightarrow} A$ with the property that $\eta_{A}^{L} e \sqsubseteq p_{\alpha} !^{A} e$ then $e$ must factor through $i$. This will show that $i$ is the lax equalizer universally setting $\eta_{A}^{L}$ less than or equal to $p_{\alpha} !^{A}$.

By change of base (to stage $E$ ) we can assume that we are given $1 \stackrel{e}{\rightarrow} A$ with the property that $\eta_{A}^{L} e \sqsubseteq p_{\alpha} !^{A} e$. By taking exponential transpose this implies that $\mathbb{S}^{A} \stackrel{\mathbb{S}^{e}}{\rightarrow} \mathbb{S}$ is less than or equal to $\alpha$ in the order enrichment. Say the subobject $1 \stackrel{e}{\rightarrow} A$ (which must be open since 1 is discrete) is classified by $a_{e}: A \rightarrow \mathbb{S}$, i.e. $a_{e}=\exists_{e}(1)$. Therefore since $1=\mathbb{S}^{e}\left(a_{e}\right)$ we have that $1=\alpha\left(a_{e}\right)=\exists_{A}\left(a \sqcap a_{e}\right)$. Now consider the pullback square

$$
\begin{array}{ccccc}
I_{0} & \stackrel{p}{\rightarrow} & a & \\
i_{0} \downarrow & & \downarrow & \\
1 & \stackrel{e}{\rightarrow} & A & &
\end{array}
$$

Since $i_{0}$ factors as ! ${ }^{A} i p$, we must have that $\exists_{I_{0}}=\exists_{A} \exists_{i} \exists_{p}$ (as the left adjoint to $\mathbb{S}^{i_{o}}=\mathbb{S}^{p} \mathbb{S}^{i} \mathbb{S}^{A}$ is unique and certainly $p$ is open as it is the pullback of the open e). But then

$$
\begin{aligned}
\exists_{I_{0}}(1) & =\exists_{A} \exists_{i} \exists_{p}(1) \\
& =\exists_{A} \exists_{i} \exists_{p}\left(\mathbb{S}^{i_{o}} 1\right) \\
& =\exists_{A} \exists_{i} \mathbb{S}^{i} \exists_{e}(1) \\
& =\exists_{A}\left(a \sqcap a_{e}\right) \\
& =1,
\end{aligned}
$$

where step $(x)$ is by Beck-Chevalley of the pullback square $(+)$. But then, by Lemma 4.1.5, we have that $i_{0}$ is an isomorphism which shows that $a_{e}=$ $a \sqcap a_{e}$; since this is sufficient to show that $e$ factors through $i$ as required we are done.

For discrete $A$ therefore we have an order isomorphism between open subobjects of $A$ and join semilattice homomorphisms $\mathbb{S}^{A} \rightarrow \mathbb{S}$. The next lemma clarifies how this order isomorphism works, in other words it describes how the order isomorphism $(*)$, recalled above, specialises when $X$ is restricted to being a discrete object. 
Lemma 4.2.3. If $A$ is a discrete object of $\mathcal{C}$ then

$$
\begin{aligned}
\mathcal{C}(A, \mathbb{S}) & \rightarrow \sqcup-\operatorname{SLat}\left[\mathbb{S}^{A}, \mathbb{S}\right] \\
a & \longmapsto \exists_{A}(a \sqcap(-))
\end{aligned}
$$

is an order isomorphism whose inverse sends any $\alpha: \mathbb{S}^{A} \rightarrow \mathbb{S}$ to $\alpha\left\ulcorner\Delta_{A}\right\urcorner$ where $\left\ulcorner\Delta_{A}\right\urcorner: A \rightarrow \mathbb{S}^{A}$ is the exponential transpose of the diagonal.

Proof. The proof is essentially an application of our description of the order isomorphism $(*)$ which we have recalled above. If $a \stackrel{i}{\hookrightarrow} A$ is open, then the corresponding join semilattice homomorphism under $(*)$ is the map $\exists_{a} \mathbb{S}^{i}$; but $\exists_{a}$ factors as $\exists_{A} \exists_{i}$ (by uniqueness of left adjoints since $!^{a}$ factors as $!^{A} i$ ) and $\left.\exists_{i} \mathbb{S}^{i}=a \sqcap()_{-}\right)$; so the join semilattice homomorphism corresponding to $a$ is $\exists_{A}(a \sqcap(-))$.

In the other direction, given a join semilattice homomorphism $\alpha: \mathbb{S}^{A} \rightarrow \mathbb{S}$, we have established in the previous lemma that the corresponding weakly closed map, $i: X_{0} \hookrightarrow A$ say, is open. To complete this proof we must show that $\exists_{i}(1)=$ $\alpha\left\ulcorner\Delta_{A}\right\urcorner$. Firstly note that by our explicit description of $(*)$ recalled above that

$$
\alpha=\exists_{X_{0}} \mathbb{S}^{i} .
$$

But, further, $i$ factors as $X_{0} \stackrel{\left(i, I d_{X_{0}}\right)}{\hookrightarrow} A \times X_{0} \stackrel{\pi_{2}}{\longrightarrow} A$ where $\pi_{2}$ is open since it is the pullback of the open $!^{X_{0}}$. The proof therefore reduces to showing $\exists_{\pi_{2}} \exists_{\left(i, I d_{X_{0}}\right)}(1)=\exists_{X_{0}} \mathbb{S}\left\ulcorner\left\ulcorner\Delta_{A}\right\urcorner\right.$. Now by Beck-Chevalley applied to the pullback square

$$
\begin{array}{ccc}
X_{0} & \stackrel{i}{\rightarrow} & A \\
\left(i, I d_{X_{0}}\right) \downarrow & & \downarrow \Delta_{A} \\
X_{0} \times A & \stackrel{i \times I d_{A}}{\rightarrow} & A \times A
\end{array}
$$

(and using the fact that $\mathbb{S}^{i}(1)=1$ ) we are in fact reduced to checking that

$$
\exists_{\pi_{2}} \mathbb{S}^{i \times I d_{A}}\left(\exists_{\Delta_{A}}(1)\right)=\exists_{X_{0}} \mathbb{S}^{i}\left\ulcorner\Delta_{A}\right\urcorner .
$$

However this is immediate by taking exponential transpose since we known that (Lemma 4.1.2) $\exists_{\pi_{2}}=\exists_{X_{0}}^{A}$ because $X_{0}$ is open.

Proposition 4.2.4. If $A$ is discrete then the presheaf $\mathbb{S}^{A}$ is representable and is naturally isomorphic to $P_{L}(A)$.

Proof. The proposition is equivalent to the assertion that the exponential $\mathbb{S}^{A}$ exists in $\mathcal{C}$ and is isomorphic to $P_{L}(A)$.

But for any object $X$ of $\mathcal{C}$ we have

$$
\begin{aligned}
\mathcal{C}(X \times A, \mathbb{S}) & \cong \mathcal{C} / X\left(A_{X}, \mathbb{S}_{X}\right) \\
& \cong\left\{I \hookrightarrow A_{X} \text { open relative to } X\right\} \\
& \cong \mathcal{C} / X\left(1, P_{L}^{X}\left(A_{X}\right)\right) \\
& \cong \mathcal{C}\left(X, P_{L}(A)\right)
\end{aligned}
$$


The second to last line uses the previous lemmas carried out in the slice $\mathcal{C} / X$. The last line is because the lower power monad is slice stable.

There is therefore an isomorphism in $\mathcal{C}$ from $P_{L}(A)$ to $\mathbb{S}^{A}$ for discrete objects $A$. In later sections we will need the following explicit description of this isomorphism.

Lemma 4.2.5. (a) The natural order isomorphism $\mathcal{C}\left(X, P_{L}(A)\right) \rightarrow \mathcal{C}\left(X, \mathbb{S}^{A}\right)$ established in the previous proposition sends any $p_{\alpha}: X \rightarrow P_{L}(A)$ to the double exponential transpose of

$$
A \stackrel{\left\ulcorner\Delta_{A}\right\urcorner}{\longrightarrow} \mathbb{S}^{A} \stackrel{\alpha}{\longrightarrow} \mathbb{S}^{X} .
$$

(b) The isomorphism $\phi_{A}: P_{L}(A) \rightarrow \mathbb{S}^{A}$ established in the previous proposition is given by the double exponential transpose of

$$
A \stackrel{\left\ulcorner\Delta_{A}\right\urcorner}{\longrightarrow} \mathbb{S}^{A} \stackrel{\vartheta_{A}}{\longrightarrow} \mathbb{S}^{P_{L}(A)} .
$$

Proof. Firstly (b) is immediate from (a) since the $P_{L}(A) \rightarrow \mathbb{S}^{A}$ is the mate of the identity on $P_{L}(A)$ under $\mathcal{C}\left(X, P_{L}(A)\right) \cong \mathcal{C}\left(X, \mathbb{S}^{A}\right)$ and the identity on $P_{L}(A)$ is equal to $p_{\diamond A}$.

For (a) this is just from the previous lemma by slice stability.

\subsection{Relational composition}

In this subsection we describe the order isomorphism just established in terms of axiomatic relational composition. It seems that the most appropriate definition of relational composition is found by not restricting to discrete objects but by only making the minimal (and surely necessary) assumption that the object that is both codomain and domain in the definition of composition is open. Although the operation is then only partially defined it has all the right properties.

Definition 4.3.1. If $A$ is an open object of $\mathcal{C}$ and $R_{1}$ an open of $W \times A$ and $R_{2}$ an open of $A \times X$ then define $R_{1} ; R_{2}$, an open of $W \times X$, by

$$
R_{1} ; R_{2}=\exists_{\pi_{13}}\left(\mathbb{S}^{\pi_{12}} R_{1} \sqcap \mathbb{S}^{\pi_{23}} R_{2}\right) .
$$

Our first observation is that this definition makes sense: $\exists_{\pi_{13}}: \mathbb{S}^{W \times A \times X} \rightarrow$ $\mathbb{S}^{W \times X}$ exists since $!^{A}: A \rightarrow 1$ is an open map and therefore so too is $\pi_{13}$ : $W \times A \times X \rightarrow W \times X$ as it is the pullback of $!^{A}$ along $!^{W \times X}$.

Our second observation is that if $W, A$ and $X$ are discrete then this definition recovers relational composition. This is because $\exists_{\pi_{13}}$ defines image factorization in the category of discrete objects relative to $\mathcal{C}$.

Finally we must of course check that:

Lemma 4.3.2. Where; is defined it is associative.

Proof. Say $R_{1}$ is an open of $W \times A, R_{2}$ is an open of $A \times B$ and $R_{3}$ is an open of $B \times Y$ where $A$ and $B$ are open objects of $\mathcal{C}$. We must check that

$$
\left(R_{1} ; R_{2}\right) ; R_{3}=R_{1} ;\left(R_{2} ; R_{3}\right)
$$


But the LHS of $(*)$ is

$$
\begin{aligned}
& \exists_{\pi_{13}}\left(\mathbb{S}^{\pi_{12}} \exists_{\pi_{13}}\left(\mathbb{S}^{\pi_{12}} R_{1} \sqcap \mathbb{S}^{\pi_{23}} R_{2}\right) \sqcap \mathbb{S}^{\pi_{23}} R_{3}\right) \\
= & \exists_{\pi_{13}}\left(\exists_{\pi_{134}} \mathbb{S}^{\pi_{123}}\left(\mathbb{S}^{\pi_{12}} R_{1} \sqcap \mathbb{S}^{\pi_{23}} R_{2}\right) \sqcap \mathbb{S}^{\pi_{23}} R_{3}\right) \\
= & \exists_{\pi_{13}} \exists_{\pi_{134}}\left(\left(\mathbb{S}^{\pi_{12}} R_{1} \sqcap \mathbb{S}^{\pi_{23}} R_{2}\right) \sqcap \mathbb{S}^{\pi_{134}} \mathbb{S}^{\pi_{23}} R_{3}\right) \\
= & \exists_{\pi_{14}}\left(\mathbb{S}^{\pi_{12}} R_{1} \sqcap \mathbb{S}^{\pi_{23}} R_{2} \sqcap \mathbb{S}^{\pi_{34}} R_{3}\right)
\end{aligned}
$$

where the first line is by Beck-Chevalley for the pullback square

$$
\begin{array}{ccc}
W \times A \times B \times Y & \stackrel{\pi_{123}}{\longrightarrow} & W \times A \times B \\
\pi_{134} \downarrow & & \downarrow \pi_{13} \\
W \times B \times Y & \stackrel{\pi_{12}}{\longrightarrow} & W \times B
\end{array}
$$

The second line exploits the fact that $\pi_{134}: W \times A \times B \times Y \rightarrow W \times B \times Y$ is open (it is the pullback of the open $\pi_{13}$ ) and so satisfies Frobenius reciprocity. The last line is because $\pi_{14}: W \times A \times B \times Y \rightarrow W \times Y$ factors as $W \times A \times B \times Y \stackrel{\pi_{134}}{\longrightarrow}$ $W \times B \times Y \stackrel{\pi_{13}}{\rightarrow} W \times Y$; therefore $\mathbb{S}^{\pi_{134}} \mathbb{S}^{\pi_{13}}=\mathbb{S}^{\pi_{14}}$ and so $\exists_{\pi_{13}} \exists_{\pi_{134}}=\exists_{\pi_{14}}$ by uniqueness of left adjoints.

Symmetrically the RHS of $(*)$ can be reduced to $\exists_{\pi_{14}}\left(\mathbb{S}^{\pi_{12}} R_{1} \sqcap \mathbb{S}^{\pi_{23}} R_{2} \sqcap\right.$ $\mathbb{S}^{\pi_{34}} R_{3}$ ) and so the proof is complete.

Beyond helping with spatial intuitions our relational composition operation is also key as it allows us to be more explicit about the order isomorphism $\mathcal{C}(X \times A, \mathbb{S}) \cong \sqcup-\mathbf{S L a t}\left(\mathbb{S}^{A}, \mathbb{S}^{X}\right)$ established above.

Lemma 4.3.3. If $A$ is a discrete object of $\mathcal{C}$ then the map

$$
\begin{aligned}
\mathcal{C}(A \times X, \mathbb{S}) & \rightarrow \sqcup-\operatorname{SLat}\left[\mathbb{S}^{A}, \mathbb{S}^{X}\right] \\
R & \longmapsto \alpha_{R}
\end{aligned}
$$

with $\alpha_{R}$ defined by $\left[\alpha_{R}\right]_{W}(I)=I ; R$, is an order isomorphism whose inverse sends any $\alpha: \mathbb{S}^{A} \rightarrow \mathbb{S}^{X}$ to the exponential transpose of $\alpha\left\ulcorner\Delta_{A}\right\urcorner: A \rightarrow \mathbb{S}^{X}$.

In other words for every open relation $R$ on $A \times X,\ulcorner R\urcorner: A \rightarrow \mathbb{S}^{X}$ factors as $\alpha_{R}\left\ulcorner\Delta_{A}\right\urcorner$ where $\alpha_{R}$ is defined in terms of relational composition.

Proof. Because of (a) of Lemma 4.2.5 we must but show that the image of $R$ under the isomorphism of that lemma is indeed $\alpha_{R}$. It is sufficient to check that

$$
\ulcorner R\urcorner=\alpha_{R}\left\ulcorner\Delta_{A}\right\urcorner
$$

which is the same as checking that $\Delta_{R} ; R=R$. This is true by change of base since $\Delta_{A_{X}}$ is the identity for relational composition in $\operatorname{Dis}_{\mathcal{C} / X}$. Alternatively 
we can use Beck-Chevalley again:

$$
\begin{aligned}
\Delta_{R} ; R & =\exists_{\pi_{13}}\left(\mathbb{S}^{\pi_{12}} \Delta_{A} \sqcap \mathbb{S}^{\pi_{23}} R\right) \\
& =\exists_{\pi_{13}}\left(\mathbb{S}^{\pi_{12}} \exists_{\Delta_{A}}\left(1_{\mathbb{S}^{A}}\right) \sqcap \mathbb{S}^{\pi_{23}} R\right) \\
& =\exists_{\pi_{13}}\left(\exists_{\Delta_{A} \times I d_{X}} \mathbb{S}^{\pi_{1}}\left(1_{\mathbb{S}^{A}}\right) \sqcap \mathbb{S}^{\pi_{23}} R\right) \quad(\mathrm{a}) \\
& =\exists_{\pi_{13}} \exists_{\Delta_{A} \times I d_{X}}\left(\mathbb{S}^{\pi_{1}}\left(1_{\mathbb{S}^{A}}\right) \sqcap \mathbb{S}^{\Delta_{A} \times I d_{X}} \mathbb{S}^{\pi_{23}} R\right) \\
& =\exists_{I d_{A \times X}}\left(1_{\mathbb{S}^{A} \times X} \sqcap R\right) \\
& =R
\end{aligned}
$$

where (a) is by Beck-Chevalley on the pullback square

$$
\begin{array}{ccc}
A \times X & \stackrel{\pi_{1}}{\rightarrow} & A \\
\Delta_{A} \times I d_{X} \downarrow & & \downarrow \Delta_{A} \\
A \times A \times X & \stackrel{\pi_{12}}{\rightarrow} & A \times A
\end{array}
$$

and (b) is because $\Delta_{A} \times I d_{X}$ is open since it is the pullback of the open map $\Delta_{A}$.

Corollary 4.3.4. Where it is defined relational composition is mapped to morphism composition under the isomorphism of the lemma.

Proof. This is immediate since the lemma describes any join semilattice corresponding to a relation in terms of relational composition and we have already checked that relational composition is associative.

The following result makes use of this explicit description of the order isomorphism in terms of relational composition. It proves, for discrete objects at least, that product in $\mathcal{C}$ is given by join semilattice tensor in $\mathcal{C}_{P_{L}}^{o p}$. In the case that $\mathcal{C}=$ Loc this is the equivalent to the basic observation that locale product is given by suplattice tensor.

Proposition 4.3.5. If $A$ and $B$ are two discrete objects of $\mathcal{C}$ then,

(i) the map $\otimes: \mathbb{S}^{A} \times \mathbb{S}^{B} \rightarrow \mathbb{S}^{A \times B}$ defined by $\otimes=\Pi_{\mathbb{S} A \times B}\left(\mathbb{S}^{\pi_{1}} \times \mathbb{S}^{\pi_{2}}\right)$ is universally join bilinear,

(ii) the map $\overline{\nabla_{A \times B} \otimes}: P_{L}(A \times B) \rightarrow \mathbb{P}(A+B)$ defined as the double exponential transpose of $\mathbb{S}^{A} \times \mathbb{S}^{B} \stackrel{\otimes}{\rightarrow} \mathbb{S}^{A \times B} \stackrel{\diamond_{A \times B}}{\longrightarrow} \mathbb{S}^{P_{L}(A \times B)}$ is a monomorphism.

Proof. (i) We must check that for any join semilattice homomorphism $\mathbb{S}^{A \times B} \rightarrow$ $\mathbb{S}^{Y}$ that precomposition with $\otimes$ induces an order isomorphism between $\sqcup-$ $\operatorname{SLat}\left(\mathbb{S}^{A \times B}, \mathbb{S}^{Y}\right)$ and

$$
\sqcup-\operatorname{Bilinear}\left(\mathbb{S}^{A} \times \mathbb{S}^{B}, \mathbb{S}^{Y}\right) .
$$

By change of base we can assume that $Y=1$. But we have the following series of order isomorphisms:

$$
\begin{aligned}
\sqcup-\operatorname{Bilinear}\left(\mathbb{S}^{A} \times \mathbb{S}^{B}, \mathbb{S}\right) & \cong \sqcup-\operatorname{SLat}\left(\mathbb{S}^{A}, P_{L}(B)\right) \\
& \cong \sqcup-\operatorname{Sat}\left(\mathbb{S}^{A}, \mathbb{S}^{B}\right) \\
& \cong \mathcal{C}(A \times B, \mathbb{S}) \\
& \cong \sqcup-\operatorname{SLat}\left(\mathbb{S}^{A \times B}, \mathbb{S}\right)
\end{aligned}
$$


where the first line is by exponential transpose and the definition of $P_{L}(B)$ as a subobject of $\mathbb{P}(B)$. Therefore we have but to check that in the reverse direction (i.e. from $\sqcup-S \operatorname{Lat}\left(\mathbb{S}^{A \times B}, \mathbb{S}\right)$ to $\sqcup-\operatorname{Bilinear}\left(\mathbb{S}^{A} \times \mathbb{S}^{B}, \mathbb{S}\right)$ ) any join semilattice $\beta: \mathbb{S}^{A \times B} \rightarrow \mathbb{S}$ is mapped to $\beta \otimes$. From above such a $\beta$ is given by $\exists_{A \times B}\left(R_{\beta} \sqcap\left({ }_{-}\right)\right)$ for some unique open $R_{\beta}$ of $A \times B$; the image of $\beta$ in $\sqcup-S \operatorname{Lat}\left(\mathbb{S}^{A}, \mathbb{S}^{B}\right)$ is therefore $\alpha_{R_{\beta}}$. On the other hand the image of $\beta \otimes$ in $\sqcup-S L a t\left(\mathbb{S}^{A}, \mathbb{S}^{B}\right)$ under the above order isomorphism is the composite

$$
\mathbb{S}^{A} \stackrel{\ulcorner\beta \otimes\urcorner}{\longrightarrow} P_{L}(B) \stackrel{\phi_{B}}{\longrightarrow} \mathbb{S}^{B}
$$

Our proof of part (i) will then be complete provided that we can prove that these two join semilattice homomorphism from $\mathbb{S}^{A}$ to $\mathbb{S}^{B}$ are equal. This can be achieved by checking for any $Z \stackrel{z}{\rightarrow} \mathbb{S}^{A}$ we have $\alpha_{R_{\beta}} z=\phi_{B}\ulcorner\beta \otimes\urcorner z$; but in fact we can assume $Z=1$ (and so $z=I$ for some open $I$ of $A$ ) by applying a change of base argument. Now $\ulcorner\beta \otimes\urcorner(I)$ is the point of $P_{L}(B)$ corresponding to the map $\mathbb{S}^{B} \stackrel{\beta(I \otimes-)}{\longrightarrow} \mathbb{S}$, i.e. to $\mathbb{S}^{B} \stackrel{\exists_{A \times B}\left(R_{\beta} \sqcap I \otimes(-)\right)}{\longrightarrow} \mathbb{S}$. Its image under the isomorphism $P_{L}(B) \rightarrow \mathbb{S}^{B}$ is the open $\exists_{A \times B}\left(R_{\beta} \sqcap I \otimes\left(\left\ulcorner\Delta_{B}\right\urcorner\right)\right)$ by lemma 4.2 .5 , which we can see is equal to $\exists_{B} \exists_{\pi_{2}}\left(R_{\beta} \sqcap \mathbb{S}^{\pi_{1}} I \sqcap \mathbb{S}^{\pi_{2}}\left(\left\ulcorner\Delta_{B}\right\urcorner\right)\right)$ by using the definition of $\otimes$ and the fact that $\exists_{A \times B}=\exists_{B} \exists_{\pi_{2}}$ since $!^{A \times B}=!^{B} \pi_{2}$ for $\pi_{2}: A \times B \rightarrow B$. Since $\pi_{2}$ is open (as it is the pullback of the open map $!^{A}$ ) we have that this is equal to

$$
\begin{aligned}
& \exists_{B}\left[\exists_{\pi_{2}}\left(R_{\beta} \sqcap \mathbb{S}^{\pi_{1}} I\right) \sqcap\left(\left\ulcorner\Delta_{B}\right\urcorner\right)\right) \\
= & \exists_{\pi_{2}}\left(R_{\beta} \sqcap \mathbb{S}^{\pi_{1}} I\right) .
\end{aligned}
$$

But we are then done since $\alpha_{R_{\beta}} I$ is the open $I ; R_{\beta}=\exists_{\pi_{2}}\left(R_{\beta} \sqcap \mathbb{S}^{\pi_{1}} I\right)$.

(ii) is immediate given the universal property of $\otimes$ just established. Note that the double exponential transpose of $\mathbb{S}^{A \times B} \stackrel{\vartheta_{A \times B}}{\longrightarrow} \mathbb{S}^{P_{L}(A \times B)}$ is, by definition, the regular monomorphism $j_{A \times B}^{L}: P_{L}(A \times B) \hookrightarrow \mathbb{P}(A \times B)$.

An appendix has been included examining the additional assumption (which is made in [T06]) that $\otimes: \mathbb{S}^{X} \times \mathbb{S}^{Y} \rightarrow \mathbb{S}^{X \times Y}$ is universal join bilinear for all objects $X$ and $Y$. The appendix shows that this additional assumption is slice stable in the presence of the other axioms. It also includes a conjecture concerning the axiomatic account of localic subgroupoids.

\subsection{The ideal completion of a preorder}

In this subsection we introduce the ideal completion of a preorder and develop some basic properties of it. The main result is that this ideal completion construction is a specialisation of a construction given [T05].

Definition 4.4.1. A preorder relative to $\mathcal{C}$ is a discrete object $B$ together with a open relation $\preceq_{B} \hookrightarrow B \times B$ which satisfies

$$
\begin{array}{rll}
\text { (i) } \Delta_{B} & \sqsubseteq & \preceq_{B}, \text { and } \\
\text { (ii) } \preceq_{B} ; \preceq_{B} & \sqsubseteq & \preceq_{B} .
\end{array}
$$


Lemma 4.4.2. The data for a preorder is equivalently a pair $\left(B, \alpha: \mathbb{S}^{B} \rightarrow \mathbb{S}^{B}\right)$ with the properties that (i) $B$ is discrete, (ii) $\alpha$ is a join semilattice homomorphism (iii) $I d_{\mathbb{S}^{B}} \sqsubseteq \alpha$ and (iv) $\alpha$ is idempotent.

Proof. This is immediate from our observations about the order isomorphism between open subobjects of $B \times B$ and join semilattice homomorphisms $\mathbb{S}^{B} \rightarrow$ $\mathbb{S}^{B}$; in particular the observation that relational composition maps to morphism composition.

If $B$ is a preorder then we use the notation $R_{B} \hookrightarrow B \times B \times B$ (and $B \times$ $B \times B \stackrel{R_{B}}{\rightarrow}$ ) to denote the open subobject $\pi_{12}^{*}\left(\preceq_{B}\right) \wedge \pi_{13}^{*}\left(\preceq_{B}\right)$ (equivalently, the open $\left.\mathbb{S}^{\pi_{12}}\left(\preceq_{B}\right) \sqcap \mathbb{S}^{\pi_{13}}\left(\preceq_{B}\right)\right)$. Set theoretically we are of course thinking $R_{B}=\left\{\left(b_{1}, b_{2}, b_{3}\right) \mid b_{1} \succeq_{B} b_{2}\right.$ and $\left.b_{1} \succeq_{B} b_{3}\right\}$.

Definition 4.4.3. If $B$ is a preorder relative to $\mathcal{C}$ then define $\operatorname{Idl}(B) \stackrel{i_{B}}{\hookrightarrow} \mathbb{S}^{B}$ to be the intersection (=pullback) of the equalizer

$$
A \sqcap \hookrightarrow \mathbb{S}^{B} \underset{\otimes \Delta_{\mathbb{S} B}}{\stackrel{\alpha_{R_{B}}}{\longrightarrow}} \mathbb{S}^{B \times B}
$$

and

$$
A^{1} \hookrightarrow \mathbb{S}^{B} \underset{1_{\mathbb{S}} !^{B}}{\stackrel{\Xi_{B}}{\longrightarrow}} \mathbb{S} .
$$

Capturing the usual spatial notion we have that, by definition, an open $I$ of a preorder $B$ is said to be an ideal provided that

(i) $\exists_{B}(I)=1_{\mathbb{S}} \quad$ - " $I$ is non-empty",

(ii) $I ; \succeq_{B}=I \quad-$ " $I$ is lower closed"; and

(iii) $I \otimes I \sqsubseteq I ; R_{B} \quad-$ " $I$ is directed".

Note that condition (ii) is equivalent to $I ; \succeq_{B} \sqsubseteq I$ since $\Delta_{B} \sqsubseteq \preceq_{B}$ by definition of preorder. The next proposition justifies our notation $\operatorname{Idl}(B)$ since it shows that the points of $\operatorname{Idl}(B)$ are in order isomorphism with the ideals of $B$.

Proposition 4.4.4. For any open $I$ of a preorder $B, I: 1 \rightarrow \mathbb{S}^{B}$ factors via $\operatorname{Idl}(B) \stackrel{i_{B}}{\hookrightarrow} \mathbb{S}^{B}$ if and only if $I$ is an ideal of $B$.

Proof. This is essentially by the construction of $\operatorname{Idl}(B)$. The only missing step is a proof that $I$ is lower closed (equivalently $I ; \succeq_{B} \sqsubseteq I$ ) if and only if $I ; R_{B} \sqsubseteq I \otimes I$.

Firstly, say that $I ; R_{B} \sqsubseteq I \otimes I$, then by application of $\mathbb{S}^{\Delta_{B}}$ we have that

$$
\mathbb{S}^{\Delta_{B}} I ; R_{B} \sqsubseteq I
$$

since $I \otimes I=\mathbb{S}^{\pi_{1}} I \sqcap \mathbb{S}^{\pi_{2}} I$ (and certainly $\mathbb{S}^{\Delta_{B}} \mathbb{S}^{\pi_{1}}=\mathbb{S}^{\Delta_{B}} \mathbb{S}^{\pi_{2}}=I d_{\mathbb{S}^{B}}$ ). But $\mathbb{S}^{\Delta_{B}} I ; R_{B}$ is equal to

$$
\mathbb{S}^{\Delta_{B}} \exists_{\pi_{23}}\left(\mathbb{S}^{\pi_{1}} I \sqcap \mathbb{S}^{\pi_{12}} \succeq_{B} \sqcap \mathbb{S}^{\pi_{13}} \succeq_{B}\right)
$$


(where $\pi_{1}: B \times B \times B \rightarrow B$ ) which reduces to

$$
\exists_{\pi_{2}}\left(\mathbb{S}^{\pi_{1}} I \sqcap \succeq_{B}\right)
$$

by Beck-Chevalley applied to the pullback square

$$
\begin{array}{ccc}
B \times B & \stackrel{I d_{B} \times \Delta_{B}}{\longrightarrow} & B \times B \times B \\
\pi_{2} \downarrow & & \downarrow \pi_{23} \\
B & \stackrel{\Delta_{B}}{\longrightarrow} & B \times B
\end{array}
$$

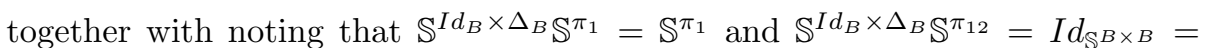

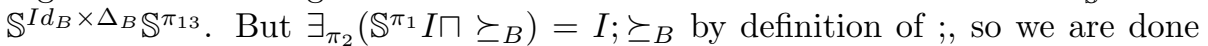
checking that $I ; R_{B} \sqsubseteq I \otimes I$ implies that $I$ is lower closed.

Secondly, in the other direction, say $I$ is lower closed, so we have that $I ; \succeq_{B} \sqsubseteq$ $I$, i.e. $\exists_{\pi_{2}}\left(\mathbb{S}^{\pi_{1}} I \sqcap \succeq_{B}\right) \sqsubseteq I$. Then, since $\exists_{\pi_{2}}$ is left adjoint to $\mathbb{S}^{\pi_{2}}$ we have that $\mathbb{S}^{\pi_{1}} I \sqcap \succeq_{B} \sqsubseteq \mathbb{S}^{\pi_{2}} I$. To show that $I ; R_{B} \sqsubseteq I \otimes I$ we are required to show

$$
\exists_{\pi_{23}}\left(\mathbb{S}^{\pi_{1}} I \sqcap \mathbb{S}^{\pi_{12}} \succeq_{B} \sqcap \mathbb{S}^{\pi_{13}} \succeq_{B}\right) \sqsubseteq \mathbb{S}^{\pi_{1}} I \sqcap \mathbb{S}^{\pi_{2}} I .
$$

We will just show that

$$
\exists_{\pi_{23}}\left(\mathbb{S}^{\pi_{1}} I \sqcap \mathbb{S}^{\pi_{12}} \succeq_{B} \sqcap \mathbb{S}^{\pi_{13}} \succeq_{B}\right) \sqsubseteq \mathbb{S}^{\pi_{1}} I
$$

since proof of the same inequality but with $\pi_{2}$ on the RHS rather than $\pi_{1}$ is symmetric. However this is immediate by noting that $\exists_{\pi_{23}} \dashv \mathbb{S}^{\pi_{23}}$, that $\mathbb{S}^{\pi_{23}} \mathbb{S}^{\pi_{1}}$ factors as $\mathbb{S}^{\pi_{12}} \mathbb{S}^{\pi_{2}}$ and that $\mathbb{S}^{\pi_{1}} I$ (for $\pi_{1}: B \times B \times B \rightarrow B$ ) is equal to $\mathbb{S}^{\pi_{12}} \mathbb{S}^{\pi_{1}} I$, since then

$$
\begin{aligned}
\mathbb{S}^{\pi_{1}} I \sqcap \mathbb{S}^{\pi_{12}} \succeq_{B} \sqcap \mathbb{S}^{\pi_{13}} \succeq_{B} & \sqsubseteq \mathbb{S}^{\pi_{12}}\left(\mathbb{S}^{\pi_{1}} I \sqcap \succeq_{B}\right) \\
& \sqsubseteq \mathbb{S}^{\pi_{12}} \mathbb{S}^{\pi_{2}} I=\mathbb{S}^{\pi_{23}} \mathbb{S}^{\pi_{1}} I .
\end{aligned}
$$

We now relate the construction of the ideal completion of a preorder to a more general construction given in [T05]. The reason for checking that our construction is the same is that we will need some of the known properties of the more general construction in our applications.

Our Axiom 8 asserts that $P_{L}$ is a KZ-monad and [T05] shows that this implies that such inflationary idempotents split. With an assumption that the lower power monad is $\mathrm{KZ}$, given any object $X$ and an inflationary and idempotent join semilattice homomorphism $\alpha: \mathbb{S}^{X} \rightarrow \mathbb{S}^{X}$ we can find an object $E$ and join semilattice homomorphisms $\beta: \mathbb{S}^{X} \rightarrow \mathbb{S}^{E}$ and $\gamma: \mathbb{S}^{E} \rightarrow \mathbb{S}^{X}$ such that $\gamma \beta=\alpha$ and $\beta \gamma=I d_{\mathbb{S}^{E}}$. In fact [T05] shows that $\gamma$ is a distributive lattice homomorphism; this fact will play a key role later. We now recall how $E$ is constructed. Consider the morphisms

$$
\begin{array}{ll}
\varepsilon^{\sqcap}: & \mathbb{S}^{X} \times \mathbb{S}^{X} \stackrel{\diamond_{X \times \diamond}}{\longrightarrow} \mathbb{S}^{P_{L} X} \times \mathbb{S}^{P_{L} X} \stackrel{\sqcap}{\longrightarrow} \mathbb{S}^{P_{L} X} \text { and } \\
\delta^{\sqcap}: & \mathbb{S}^{X} \times \mathbb{S}^{X} \stackrel{\alpha \times \alpha}{\longrightarrow} \mathbb{S}^{X} \times \mathbb{S}^{X} \stackrel{\sqcap}{\longrightarrow} \mathbb{S}^{X} \stackrel{\diamond_{X}}{\longrightarrow} \mathbb{S}^{P_{L} X} .
\end{array}
$$


Let $E^{\sqcap} \hookrightarrow P_{L} X$ be the equalizer in $\mathcal{C}$ of their double exponential transposes, i.e. of

$$
P_{L} X \underset{\overline{\delta \sqcap}}{\stackrel{\bar{\epsilon}}{\longrightarrow}} \mathbb{P}(X+X) .
$$

Further consider the morphisms

$$
\begin{array}{ll}
\varepsilon^{1}: & 1 \stackrel{1}{\rightarrow} \mathbb{S}^{P_{L} X} \text { and } \\
\delta^{1}: & 1 \stackrel{1}{\longrightarrow} \mathbb{S}^{X} \stackrel{\diamond_{X}}{\longrightarrow} \mathbb{S}^{P_{L} X},
\end{array}
$$

and let $E^{1} \hookrightarrow P_{L} X$ be the equalizer in $\mathcal{C}$ of their exponential transposes. Take $e_{X}: E \hookrightarrow P_{L} X$ to be the intersection of $E^{1}$ and $E^{\sqcap}$. To check that this construction is indeed a generalisation of the definition just given we need to prove:

Lemma 4.4.5. $E \cong I d l(B)$ in the case that $X=B$, a preorder relative to $\mathcal{C}$, and $\alpha=\alpha \preceq_{B}$.

Proof. There is a monomorphism $\overline{\nabla_{B \times B} \otimes}: P_{L}(B \times B) \hookrightarrow \mathbb{P}(X+X)$ given by mapping any join semilattice homomorphism $\gamma: \mathbb{S}^{B \times B} \rightarrow \mathbb{S}^{Y}$ to $\mathbb{S}^{B} \times \mathbb{S}^{B} \stackrel{\otimes}{\longrightarrow}$ $\mathbb{S}^{B \times B} \stackrel{\gamma}{\rightarrow} \mathbb{S}^{Y}$. The result then follows by verifying that the equalizer diagram given in the construction of $E$ factors as the equalizer diagram given in the definition of $\operatorname{Idl}(B)$ followed by $\overline{\nabla_{B \times B} \otimes}$. Since $\overline{\nabla_{B \times B} \otimes}$ is a monomorphism this shows that the two constructions are isomorphic. In detail to complete the proof we need to check that $(\mathrm{A}) \mathbb{S}^{B} \stackrel{\cong}{\cong} P_{L} B \stackrel{\overline{\varepsilon \sqcap}}{\longrightarrow} \mathbb{P}(B+B)$ factors as

$$
\mathbb{S}^{B} \stackrel{\otimes \Delta_{\mathrm{S} B}}{\longrightarrow} \mathbb{S}^{B \times B} \cong P_{L}(B \times B) \hookrightarrow \mathbb{P}(X+X),
$$

(B) $\mathbb{S}^{B} \stackrel{\cong}{\rightrightarrows} P_{L} B \stackrel{\overline{\delta^{\sqcap}}}{\longrightarrow} \mathbb{P}(B+B)$ factors as

$$
\mathbb{S}^{B} \stackrel{\alpha_{R_{B}}}{\longrightarrow} \mathbb{S}^{B \times B} \cong P_{L}(B \times B) \hookrightarrow \mathbb{P}(X+X)
$$

and similarly for the two nullary cases.

(A)

To show (A) we check that for any $Y \stackrel{z}{\rightarrow} \mathbb{S}^{B}$,

$$
Y \stackrel{z}{\rightarrow} \mathbb{S}^{B} \stackrel{\cong}{\rightrightarrows} P_{L} B \stackrel{\overline{\varepsilon^{\sqcap}}}{\longrightarrow} \mathbb{P}(B+B)
$$

is equal to

$$
Y \stackrel{z}{\rightarrow} \mathbb{S}^{B} \stackrel{\otimes \Delta_{\mathbb{S} B}}{\longrightarrow} \mathbb{S}^{B \times B} \cong P_{L}(B \times B) \stackrel{\widehat{\vartheta}_{B \times B}}{\longrightarrow} \mathbb{P}(X+X)
$$

Since all of the constructions involved are stable under change of base we can reduce to the case that $Y=1$ and that therefore $z=I$, some open of $B$. In this case (II) corresponds to the natural transformation

$$
\mathbb{S}^{B} \times \mathbb{S}^{B} \stackrel{\otimes}{\rightarrow} \mathbb{S}^{B \times B} \stackrel{\exists_{B \times B}(I \otimes I \sqcap(-))}{\longrightarrow} \mathbb{S}
$$


since the map $\overline{\nabla_{B \times B} \otimes}$ is effectively 'precompose with $\otimes$ ' and the isomorphism $\mathbb{S}^{B \times B} \cong P_{L}(B \times B)$ sends any relation $R$ on $B \times B$ to $\exists_{B}\left(R \sqcap\left({ }_{-}\right)\right)$. (I) on the other hand corresponds (in the case $Y=1, z=I$ ) to

$$
\mathbb{S}^{B} \times \mathbb{S}^{B} \stackrel{\diamond_{X} \times \diamond_{X}}{\longrightarrow} \mathbb{S}^{P_{L} B} \times \mathbb{S}^{P_{L} B} \stackrel{\sqcap}{\rightarrow} \mathbb{S}^{P_{L} B} \stackrel{\mathbb{S}^{p_{I}}}{\rightarrow} \mathbb{S}
$$

which is equal to

$$
\mathbb{S}^{B} \times \mathbb{S}^{B} \stackrel{\exists_{B}(I \sqcap(-)) \times \exists_{B}(I \sqcap(-))}{\longrightarrow} \mathbb{S} \times \mathbb{S} \stackrel{\sqcap}{\longrightarrow} \mathbb{S}
$$

since $\mathbb{S}^{p_{I}}$ is a meet semilattice homomorphism and $\mathbb{S}^{p_{I}} \diamond_{B}=$ the join semilattice homomorphism $\mathbb{S}^{B} \rightarrow \mathbb{S}$ corresponding to $I$, which is equal to $\exists_{B}(I \sqcap(-))$ a result we have already just drawn on. To prove that (i) is equal to (ii) it is sufficient, again by change of base, to check that they are the same when precomposed with $1 \stackrel{(J, K)}{\longrightarrow} \mathbb{S}^{B} \times \mathbb{S}^{B}$ for arbitrary opens $J$ and $K$ of $B$. So a verification of (A) reduces to checking that

$$
\exists_{B \times B}(I \otimes I \sqcap J \otimes K)=\exists_{B}(I \sqcap J) \sqcap \exists_{B}(I \sqcap K)
$$

But the LHS of $(*)$ is equal to

$$
\begin{aligned}
& \exists_{B} \exists_{\pi_{1}}\left(\mathbb{S}^{\pi_{1}}(I \sqcap J) \sqcap \mathbb{S}^{\pi_{2}}(I \sqcap K)\right) \\
= & \exists_{B}\left[(I \sqcap J) \sqcap \exists_{\pi_{1}} \mathbb{S}^{\pi_{2}}(I \sqcap K)\right] \\
= & \exists_{B}\left[(I \sqcap J) \sqcap \mathbb{S}^{!^{B}} \exists_{B}(I \sqcap K)\right] \\
= & \exists_{B}(I \sqcap J) \sqcap \exists_{B}(I \sqcap K)
\end{aligned}
$$

where the first step is because $\pi_{1}: B \times B \rightarrow B$ is open (it is the pullback of the open map $!^{B}: B \rightarrow 1$ ), the second step is Beck-Chevalley applied to the pullback square

$$
\begin{array}{ccc}
B \times B & \stackrel{\pi_{2}}{\rightarrow} & B \\
\pi_{1} \downarrow & & \downarrow !^{B} \\
B & \stackrel{!^{B}}{\rightarrow} & 1
\end{array}
$$

and the third and final step is because $!^{B}$ is open. This completes our verification of $(\mathrm{A})$.

To check (B) it is sufficient to show for any $Y \stackrel{z}{\rightarrow} \mathbb{S}^{B}$ that $\overline{\nabla_{B \times B} \otimes} \alpha_{R_{B}} z=$ $\bar{\delta} z$. By change of base we can take $Y=1$ and $z=I$, an arbitrary open of $B$. Now $\overline{\nabla_{B \times B} \otimes} \alpha_{R_{B}} I$ is the natural transformation

$$
\mathbb{S}^{B} \times \mathbb{S}^{B} \stackrel{\otimes}{\longrightarrow} \mathbb{S}^{B \times B} \stackrel{\exists_{B \times B}(I ; R \sqcap(-))}{\longrightarrow} \mathbb{S}
$$

The image of $I$ under $\mathbb{S}^{B} \stackrel{\cong}{\rightrightarrows} P_{L} B \stackrel{\overline{\delta^{\sqcap}}}{\longrightarrow} \mathbb{P}(B+B)$ is

$$
\mathbb{S}^{B} \times \mathbb{S}^{B} \stackrel{\alpha{ }_{B} \times \alpha \preceq_{B}}{\longrightarrow} \mathbb{S}^{B} \times \mathbb{S}^{B} \stackrel{\sqcap}{\longrightarrow} \mathbb{S}^{B} \stackrel{\vartheta_{B}}{\longrightarrow} \mathbb{S}^{P_{L}(B)} \stackrel{\mathbb{S}^{p} I}{\longrightarrow} \mathbb{S}
$$


which is equal to

$$
\mathbb{S}^{B} \times \mathbb{S}^{B} \stackrel{\alpha_{\underline{S}_{B}} \times \alpha_{\leq_{B}}}{\longrightarrow} \mathbb{S}^{B} \times \mathbb{S}^{B} \stackrel{\square}{\longrightarrow} \mathbb{S}^{B} \stackrel{\exists_{B}(I \sqcap(-))}{\longrightarrow} \mathbb{S} .
$$

So, by change of base, to establish (B) it is sufficient to check for any opens $J, K: 1 \rightarrow \mathbb{S}^{B}$ of $B$ that

$$
\exists_{B}\left(I \sqcap\left(J ; \preceq_{B}\right) \sqcap\left(K ; \preceq_{B}\right)\right)=\exists_{B \times B}\left(I ; R_{B} \sqcap \mathbb{S}^{\pi_{1}} J \sqcap \mathbb{S}^{\pi_{2}} K\right)
$$

Since $R_{B}=\mathbb{S}^{\pi_{12}} \succeq_{B} \sqcap \mathbb{S}^{\pi_{13}} \succeq_{B}$ this becomes an application of Beck-Chevalley together with various applications of the definition of a map being open, since all the projections involved are open as they are pullbacks of the open map $!^{B}: B \rightarrow 1$. The RHS of $(*)$ is equal to

$$
\begin{aligned}
& \exists_{B \times B}\left(\exists_{\pi_{23}}\left(\mathbb{S}^{\pi_{1}} I \sqcap R_{B}\right) \sqcap \mathbb{S}^{\pi_{1}} J \sqcap \mathbb{S}^{\pi_{2}} K\right) \\
= & \exists_{B \times B}\left(\exists_{\pi_{23}}\left(\mathbb{S}^{\pi_{1}} I \sqcap \mathbb{S}^{\pi_{12}} \succeq_{B} \sqcap \mathbb{S}^{\pi_{13}} \succeq_{B}\right) \sqcap \mathbb{S}^{\pi_{1}} J \sqcap \mathbb{S}^{\pi_{2}} K\right) \\
= & \exists_{B \times B} \exists_{\pi_{23}}\left(\left(\mathbb{S}^{\pi_{1}} I \sqcap \mathbb{S}^{\pi_{12}} \succeq_{B} \sqcap \mathbb{S}^{\pi_{13}} \succeq_{B}\right) \sqcap \mathbb{S}^{\pi_{2}} J \sqcap \mathbb{S}^{\pi_{3}} K\right)
\end{aligned}
$$

since $\pi_{2}: B \times B \times B \rightarrow B$ factors as $B \times B \times B \stackrel{\pi_{23}}{\rightarrow} B \times B \stackrel{\pi_{1}}{\rightarrow} B$ and $\pi_{3}$ : $B \times B \times B \rightarrow B$ factors as $B \times B \times B \stackrel{\pi_{23}}{\longrightarrow} B \times B \stackrel{\pi_{2}}{\rightarrow} B$.

The LHS of $(*)$ is equal to

$$
\begin{gathered}
\exists_{B}\left(I \sqcap \exists_{\pi_{1}}\left(\mathbb{S}^{\pi_{2}} J \sqcap \succeq_{B}\right) \sqcap \exists_{\pi_{1}}\left(\mathbb{S}^{\pi_{2}} K \sqcap \succeq_{B}\right)\right) \\
=\exists_{B} \exists_{\pi_{1}}\left(\mathbb{S}^{\pi_{1}} I \sqcap\left(\mathbb{S}^{\pi_{2}} J \sqcap \succeq_{B}\right) \sqcap \mathbb{S}^{\pi_{1}} \exists_{\pi_{1}}\left(\mathbb{S}^{\pi_{2}} K \sqcap \succeq_{B}\right)\right) \\
=\exists_{B} \exists_{\pi_{1}}\left(\mathbb{S}^{\pi_{1}} I \sqcap\left(\mathbb{S}^{\pi_{2}} J \sqcap \succeq_{B}\right) \sqcap \exists_{\pi_{12}} \mathbb{S}^{\pi_{13}}\left(\mathbb{S}^{\pi_{2}} K \sqcap \succeq_{B}\right)\right) \\
\left.=\exists_{B} \exists_{\pi_{1}} \exists_{\pi_{12}}\left(\mathbb{S}^{\pi_{1}} I \sqcap \mathbb{S}^{\pi_{2}} J \sqcap \mathbb{S}^{\pi_{12}} \succeq_{B} \sqcap \mathbb{S}^{\pi_{3}} K \sqcap \mathbb{S}^{\pi_{12}} \succeq_{B}\right)\right)
\end{gathered}
$$

where the second last line is by Beck-Chevalley on the pullback square

$$
\begin{array}{ccc}
B \times B \times B & \stackrel{\pi_{13}}{\rightarrow} & B \times B \\
\pi_{12} \downarrow & & \downarrow \pi_{1} \\
B \times B & \stackrel{\pi_{1}}{\rightarrow} & B
\end{array}
$$

This completes a proof of (B) since $\exists_{B} \exists_{\pi_{1}} \exists_{\pi_{12}}=\exists_{B \times B} \exists_{\pi_{23}}$ as both are left adjoint to $\mathbb{S}^{B \times B \times B}: \mathbb{S} \rightarrow \mathbb{S}^{B \times B \times B}$.

Next we check that nullary cases. To check that $\exists_{B}: \mathbb{S}^{B} \rightarrow \mathbb{S}$ factors as $\mathbb{S}^{B} \cong P_{L}(B) \stackrel{\overline{\delta^{1}}}{\rightarrow} \mathbb{S}$, by change of base we are reducing to checking that $\exists_{B}(I)=\overline{\delta^{1}}\left(J \longmapsto \exists_{B}(I \sqcap J)\right)$ for any open $I$ of $B$. But $\overline{\delta^{1}}: P_{L}(B) \stackrel{\overline{\delta^{1}}}{\rightarrow} \mathbb{S}$ is the map which sends any $\alpha: \mathbb{S}^{B} \rightarrow \mathbb{S}$ to $\alpha\left(1_{\mathbb{S} B}\right)$ since it is the exponential transpose of $1 \stackrel{1_{\mathbb{S} B}}{\longrightarrow} \mathbb{S}^{B} \stackrel{\vartheta_{B}}{\longrightarrow} \mathbb{S}^{P_{L}(B)}$ and so is equal to $P_{L}(B) \hookrightarrow \mathbb{S}^{\mathbb{S}^{B}} \stackrel{\mathbb{S}^{1} \mathbb{S}^{B}}{\longrightarrow} \mathbb{S}$. Therefore $\overline{\delta^{1}}\left(J \longmapsto \exists_{B}(I \sqcap J)\right)=\exists_{B}\left(I \sqcap 1_{\mathbb{S}^{B}}\right)=\exists_{B}(I)$ and we are done checking that $\exists_{B}$ factors via $\overline{\delta^{1}}$.

That $\mathbb{S}^{B} \stackrel{\mathbb{S}_{\mathbb{S}^{\mathbb{S}^{B}}}}{\rightarrow} \mathbb{S}$ factors as $\mathbb{S}^{B} \cong P_{L}(B) \stackrel{\frac{\varepsilon^{1}}{\rightarrow}}{\rightarrow}$ is immediate since $\overline{\varepsilon^{1}}$ factors as $P_{L}(B) \stackrel{!^{P_{L}(B)}}{\rightarrow} 1 \stackrel{1_{\mathbb{S}}}{\rightarrow} \mathbb{S}$ by definition of $1_{\mathbb{S}^{P_{L}(B)}}$, so we are done checking the nullary cases and have finished the proof. 
In other words we have now checked that the more general construction of the ideal completion as given, in [T05], for any $X$ with an inflationary idempotent join semilattice $\alpha: \mathbb{S}^{X} \rightarrow \mathbb{S}^{X}$ coincides with our $\operatorname{Idl}(B)$ for the case that $X$ is a preorder $B$.

\subsection{The ideal completion of a join semilattice}

In this subsection we make the entirely routine observation that if $B$ is an internal join semilattice in $\operatorname{Dis}_{\mathcal{C}}$, and is therefore a preorder relative to $\mathcal{C}$, then the construction of $\operatorname{Idl}(B)$ can be simplified to an equalizer involving the join operation and no longer needs to be explicitly dependent on relational composition. This allows us to easily make the construction $\operatorname{Idl}\left(_{-}\right)$functorial and to give an explicit description of arbitrary maps $Y \rightarrow \operatorname{Idl}(B)$ in $\mathcal{C}$. It is the functoriality of this construction and the explicit description of its general points that is key to our proof, to follow in the final section, of Joyal and Tierney's result about the logical slice stability of locales.

We use the notation $V-$ SLat $_{\mathbf{D i s}}$ to denote the category of internal join semilattices in $\mathbf{D i s}_{\mathcal{C}}$. The ' $\mathrm{V}$ ' is just a notational convenience to help us decide the direction of the ordering on any given semilattice. For any internal join semilattice in a cartesian category, say denoted $\left(B, \vee_{B}, 0_{B}\right)$, as usual we define $\geq_{B} \hookrightarrow B \times B$ to be the equalizer of

$$
B \times B \stackrel{\stackrel{\vee_{B}}{\longrightarrow}}{\longrightarrow} B,
$$

and as in our discussion of preorders we define $R_{B} \hookrightarrow B \times B \times B$ to be the intersection $\pi_{12}^{*} \geq_{B}$ and $\pi_{13}^{*} \geq_{B}$. In fact $R_{B}$ has an alternative description in this case:

Lemma 4.5.1. For any join semilattice $\left(B, \vee_{B}, 0_{B}\right)$ in a cartesian category, $R_{B}$ is isomorphic to the pullback of $\geq_{B} \hookrightarrow B \times B$ along $I d_{B} \times \vee_{B}: B \times B \times B \rightarrow B \times B$.

Proof. Intuitively this is just the assertion that the set $\left\{\left(b_{1}, b_{2}, b_{3}\right) \mid b_{1} \geq_{B} b_{2}\right.$ and $\left.b_{1} \geq_{B} b_{3}\right\}$ is equal to $\left\{\left(b_{1}, b_{2}, b_{3}\right) \mid b_{1} \geq_{B} b_{2} \vee_{B} b_{3}\right\}$ which of course is entirely trivial. The categorical verification that the two pullbacks are isomorphic is routine from the definition of being an internal join semilattice and the definition of $\geq_{B}$ as an equalizer. Although the proof is entirely routine the diagram chase involved is slightly lengthy.

So, for join semilattices in Dis $\mathbf{C}_{\mathcal{C}}$, we have $R_{B}=\mathbb{S}^{I d_{B} \times \vee_{B}}\left(\geq_{B}\right)$.

Lemma 4.5.2. For any join semilattice $\left(B, \vee_{B}, 0_{B}\right)$ in $\operatorname{Dis}_{\mathcal{C}}, \operatorname{Idl}(B)$ is given by the intersection of the equalizer of

$$
\mathbb{S}^{B} \stackrel{\stackrel{\mathbb{S}^{\vee} B}{\longrightarrow}}{\underset{\otimes \Delta_{\mathbb{S} B}}{\longrightarrow}} \mathbb{S}^{B \times B}
$$


and the equalizer of

$$
\mathbb{S}^{B} \underset{1_{\mathbb{S}} !^{B}}{\stackrel{\mathbb{S}^{0} B}{\longrightarrow}} \mathbb{S} .
$$

Proof. We verify for arbitrary $Y \stackrel{z}{\rightarrow} \mathbb{S}^{B}$ that the arrows

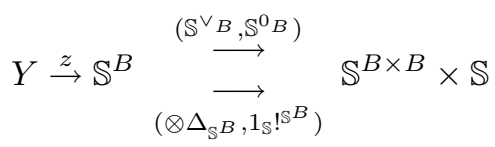

are equal if and only if the arrows

$$
Y \stackrel{z}{\rightarrow} \mathbb{S}^{B} \underset{\left(\otimes \Delta_{\mathbb{S} B}, 1_{\mathbb{S}} !^{\mathbb{S}^{B}}\right)}{\stackrel{\left(\alpha_{R_{B}}, \exists_{B}\right)}{\longrightarrow}} \mathbb{S}^{B \times B} \times \mathbb{S}
$$

are equal. As usual, by change of base we can assume that $Y=1$ and $z=I$, an open of $B$.

$(\beta) \Longrightarrow(\alpha)$

If $(\beta)$ is true then $I$ is lower closed by Proposition 4.4.4. We firstly check that $\alpha_{R_{B}}(I)=\mathbb{S}^{\vee_{B}}(I)$. We have just recalled that $R_{B}=\mathbb{S}^{I d_{B} \times \vee_{B}}\left(\geq_{B}\right)$ and so,

$$
\begin{aligned}
& \alpha_{R_{B}}(I)=\exists_{\pi_{23}}\left(R_{B} \sqcap \mathbb{S}^{\pi_{1}}(I)\right) \\
& =\exists_{\pi_{23}}\left(\mathbb{S}^{I d_{B} \times \vee_{B}}\left(\geq_{B}\right) \sqcap \mathbb{S}^{\pi_{1}}(I)\right) \\
& =\exists_{\pi_{23}}\left(\mathbb{S}^{I d_{B} \times \vee_{B}}\left(\geq_{B}\right) \sqcap \mathbb{S}^{I d_{B} \times v_{B} \mathbb{S}^{\pi_{1}}}(I)\right) \\
& =\exists_{\pi_{23}}\left(\mathbb{S}^{I d_{B} \times \vee_{B}}\left[\left(\geq_{B}\right) \sqcap \mathbb{S}^{\pi_{1}}(I)\right]\right) \\
& =\mathbb{S}^{\vee_{B}} \exists_{\pi_{2}}\left[\left(\geq_{B}\right) \sqcap \mathbb{S}^{\pi_{1}}(I)\right] \\
& =\mathbb{S}^{\vee_{B}}(I) \text {. }
\end{aligned}
$$

where the second last line is by Beck-Chevalley on the pullback square

$$
\begin{array}{ccc}
B \times B \times B & \stackrel{I d_{B} \times \vee_{B}}{\longrightarrow} & B \times B \\
\pi_{23} \downarrow & & \downarrow \pi_{2} \\
B \times B & \stackrel{\vee_{B}}{\longrightarrow} & B
\end{array}
$$

and the last line is because $I$ is lower closed.

Next we check that $\mathbb{S}^{0_{B}}(I)=\exists_{B}(I)$;

$$
\begin{aligned}
\mathbb{S}^{0_{B}}(I) & =\mathbb{S}^{0_{B}}\left(\exists_{\pi_{2}}\left(\geq_{B} \sqcap \mathbb{S}^{\pi_{1}} I\right)\right) \\
& \left.=\exists_{B} \mathbb{S}^{\left(I d_{B}, 0_{B} !^{B}\right)}\left(\geq_{B} \sqcap \mathbb{S}^{\pi_{1}} I\right)\right) \\
& =\exists_{B}(I)
\end{aligned}
$$

where the second line is by Beck-Chevalley applied to the pullback square

$\begin{array}{ccc}B & \stackrel{\left(I d_{B}, 0_{B}\right)}{\rightarrow} & B \times B \\ !^{B} \downarrow & & \downarrow \pi_{2} \\ 1 & \stackrel{0_{B}}{\longrightarrow} & B\end{array}$


and the last line is because $\mathbb{S}^{\left(I d_{B}, 0_{B} !^{B}\right)}\left(\geq_{B}\right)=1_{\mathbb{S}^{B}}$ since the pullback of $\geq_{B} \hookrightarrow$ $B \times B$ along $B \stackrel{\left(I d_{B}, 0_{B}\right)}{\longrightarrow} B \times B$ is isomorphic to the identity on $B$. This complete our proof of $(\beta) \Longrightarrow(\alpha)$.

$(\alpha) \Longrightarrow(\beta)$

Assume $(\alpha)$. Then $\mathbb{S}^{0_{B}}(I)=1_{\mathbb{S}}$. By Lemma 4.1 .4 to prove that $\exists_{B}(I)=1_{\mathbb{S}}$ it is sufficient to check that $\left\ulcorner\Delta_{B}\right\urcorner_{B} \sqsubseteq I$. The open $\left\ulcorner\Delta_{B}\right\urcorner 0_{B}$ of $B$ is the regular monomorphism $1 \stackrel{0_{B}}{\rightarrow} B$ since we have a pullback square

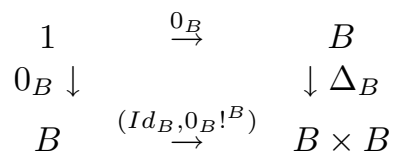

But $\mathbb{S}^{0_{B}}(I)=1_{\mathbb{S}}$ implies $1_{\mathbb{S}}: 1 \rightarrow \mathbb{S}$ factors as $1 \stackrel{0_{B}}{\rightarrow} B \stackrel{I}{\rightarrow} \mathbb{S}$, and so for any $W \stackrel{z}{\rightarrow} B$, if it factors via $1 \stackrel{0_{B}}{\rightarrow} B$ then it also factors via $I$; this shows that $\left\ulcorner\Delta_{B}\right\urcorner 0_{B} \sqsubseteq I$ (see the comments after the introduction of Axiom 3) and so $\exists_{B}(I)=1_{\mathbb{S}}$.

Finally we show that if $\mathbb{S}^{\vee_{B}}(I)=I \otimes I$ then $\alpha_{R_{B}}(I)=I \otimes I$. However the proof shown in $(\beta) \Longrightarrow(\alpha)$, that $\mathbb{S}^{\vee}(I)=\alpha_{R_{B}}(I)$ will be available provided we can check that $(\alpha)$ implies that $I$ is lower closed. To prove that $I$ is lower closed we check $\exists_{\pi_{2}}\left(\mathbb{S}^{\pi_{1}} I \sqcap \geq_{B}\right) \sqsubseteq I$, which is equivalent to checking $\mathbb{S}^{\pi_{1}} I \sqcap \geq_{B} \sqsubseteq \mathbb{S}^{\pi_{2}} I$. But $\mathbb{S}^{\vee}(I)=I \otimes I=\mathbb{S}^{\pi_{1}} I \sqcap \mathbb{S}^{\pi_{2}} I \sqsubseteq \mathbb{S}^{\pi_{2}} I$ so it is sufficient to check that

$$
\mathbb{S}^{\pi_{1}} I \sqcap \geq \geq_{B} \sqsubseteq \mathbb{S}^{\vee_{B}} I .
$$

Now for any $W \stackrel{z}{\rightarrow} B$ if $z$ factors through both (i) $\mathbb{S}^{\pi_{1}} I$ and (ii) $\geq_{B}$, then (i) $I \pi_{1} z=1_{\mathbb{S}} !^{B \times B} z$ and (ii) $\vee_{B} z=\pi_{1} z$. Putting these together we get that $I \vee_{B} z=1_{\mathbb{S}} !^{B \times B} z$ and so $z$ factors through $\mathbb{S}^{\vee_{B}} I$. This shows that $\mathbb{S}^{\pi_{1}} I \sqcap \geq_{B} \sqsubseteq$ $\mathbb{S}^{\vee} I$ and we are done.

Lemma 4.5.3. The construction given of $\operatorname{Idl}(B)$ extends to a functor $I d l$ : $\vee-\mathbf{S L a t}_{\mathbf{D i s}} \rightarrow \mathcal{C}^{o p}$ which is a subfunctor of $\mathbb{S}^{(-)}$.

Proof. Say $f: B_{1} \rightarrow B_{2}$ is a join semilattice homomorphism. Then it is clear that we have the following commuting diagram

$$
\begin{array}{lllll}
I d l\left(B_{2}\right) & \hookrightarrow & \mathbb{S}^{B_{2}} & \rightrightarrows & \mathbb{S}^{B_{2} \times B_{2}} \times \mathbb{S} \\
& & \mathbb{S}^{f} \downarrow & & \mathbb{S}^{f \times f} \times I d_{\mathbb{S}} \downarrow \\
\operatorname{Idl}\left(B_{1}\right) & \hookrightarrow & \mathbb{S}^{B_{1}} & \rightrightarrows & \mathbb{S}^{B_{1} \times B_{1}} \times \mathbb{S}
\end{array}
$$

and so there exists unique $\operatorname{Idl}(f): \operatorname{Idl}\left(B_{2}\right) \rightarrow \operatorname{Idl}\left(B_{1}\right)$ making the square on the left commute.

The following lemma clarifies why we have introduced the ideal completion of semilattices as it provides a description of the general points of $\operatorname{Idl}(B)$. 
Lemma 4.5.4. There are natural isomorphisms:

(i) $\mathcal{C}(W, \operatorname{Idl}(B)) \cong\left\{f: B \rightarrow \mathbb{S}^{W} \mid f\right.$ takes finite joins to meets $\}$ for any join semilattice $B$ and any object $W$; and,

(ii) $\mathcal{C}\left(W, \operatorname{Idl}\left(B^{o p}\right)\right) \cong \wedge-\operatorname{SLat}\left(B \rightarrow \mathbb{S}^{W}\right)$ for any meet semilattice $B$ and any object $W$.

Proof. (i) is immediate from construction. (ii) is the same assertion as (i) since $B$ is a meet semilattice if and only if $B^{o p}$ is a join semilattice.

Note that (ii) is, essentially, the well known observation from lattice theory that $\mathcal{D} B$, the set of lower closed subsets of $B$, is the free frame on the meet semilattice $B$. The reason for introducing (ii) is that in application we will want to view frames as (infinitary) algebras over meet semilattices in the familiar way. Of course we could have introduced the filter completion of a preorder exactly dually to the ideal completion and so avoided the need to introduce opposites at this point. However, although the filter completion has exactly the same mathematical content as the ideal completion, it is less familiar and so we have chosen not to introduce it.

We end this subsection with a couple of technical lemmas. They are applications of the description just given of the points of $\operatorname{Idl}\left(B^{o p}\right)$ and are required for the final section.

Lemma 4.5.5. If $A$ and $B$ are two internal meet semilattices with $A$ and $B$ discrete objects of $\mathcal{C}$ then

(i) $\mathcal{C}\left(\operatorname{Idl}\left(A^{o p}\right), \operatorname{Idl}\left(B^{o p}\right)\right) \cong\left\{B \stackrel{f}{\rightarrow} \mathbb{S}^{A} \mid f a \wedge-\right.$ SLat hom., $\left.\alpha \geq_{A} f=f\right\}$,

(ii) If $A=B$ then the image of the identity is $\left\ulcorner\geq_{B}\right\urcorner: B \rightarrow \mathbb{S}^{B}$; and,

(iii) If $h: B \rightarrow A$ is a meet semilattice homomorphism then the image of $\operatorname{Idl}(h)^{o p}: \operatorname{Idl}\left(A^{o p}\right) \rightarrow \operatorname{Idl}\left(B^{o p}\right)$ under $(i)$ is $B \stackrel{\left\ulcorner\left(h \times I d_{A}\right)^{*}\left(\geq_{A}\right)\right\urcorner}{\longrightarrow} \mathbb{S}^{A}$.

Proof. The order isomorphism follows by examining the case $W=I d l\left(A^{o p}\right)$ in $\mathcal{C}\left(W, \operatorname{Idl}\left(B^{o p}\right)\right) \cong \wedge-\operatorname{SLat}\left(B \rightarrow \mathbb{S}^{W}\right)$ and recalling that $\mathbb{S}^{I d l\left(A^{o p}\right)}$ is the split equalizer of $\mathbb{S}^{A} \underset{\alpha_{\geq_{A}}}{\stackrel{I d}{\longrightarrow}} \mathbb{S}^{A}$. This splitting, $\mathbb{S}^{I d l\left(A^{o p}\right)} \underset{\delta_{A}}{\stackrel{\varepsilon_{A}}{\hookrightarrow}} \mathbb{S}^{A}$ say, has the additional properties that

(i) $\varepsilon_{A}$ is a distributive lattice homomorphism; and,

(ii) $\delta_{A}=\mathbb{S}^{e_{A}} \diamond_{A}$, where $e_{A}: \operatorname{Idl}\left(A^{o p}\right) \hookrightarrow P_{L} A$ as introduced before lemma 4.4.5.

(See (ii) $\Longrightarrow$ (iii) of Theorem 2 in [T05] for the order dual observations.) From (i) we get that composition with $\varepsilon_{A}$ defines an order isomorphism between meet semilattice homomorphisms $B \rightarrow \mathbb{S}^{I d l\left(A^{o p}\right)}$ and meet semilattice homomorphisms $f: B \rightarrow \mathbb{S}^{A}$ such that $\alpha_{\geq_{A}} f=f$. This complete our proof of the assertion (i).

To prove that the image of the identity on $\operatorname{Idl}\left(B^{o p}\right)$ is $\left\ulcorner\geq_{B}\right\urcorner: B \rightarrow \mathbb{S}^{B}$ we must check that $B \stackrel{\widetilde{i_{B}}}{\longrightarrow} \mathbb{S}^{I d l\left(B^{o p}\right)} \stackrel{\varepsilon_{B}}{\longrightarrow} \mathbb{S}^{B}$ is equal to $\left\ulcorner\geq_{B}\right\urcorner: B \rightarrow \mathbb{S}^{B}$ where $\widetilde{i_{B}}$ is the double exponential transpose of $\operatorname{Idl}\left(B^{o p}\right) \stackrel{i_{B}}{\hookrightarrow} \mathbb{S}^{B}$. Since $\alpha_{\geq_{B}}$ factors as 
$\varepsilon_{B} \delta_{B}=\varepsilon \mathbb{S}^{e_{B}} \diamond_{B}$ and $\alpha_{\geq_{B}}\left\ulcorner\Delta_{B}\right\urcorner=\left\ulcorner\geq_{B}\right\urcorner$ it is sufficient, to complete a proof of (ii), to check that $\left.\widetilde{i_{B}}=\mathbb{S}^{e_{B}}\right\rangle_{B}\left\ulcorner\Delta_{B}\right\urcorner$. But $e_{B}$ is related to $i_{B}$ by

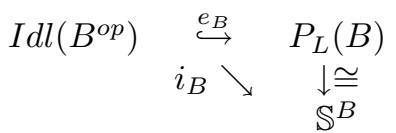

where the isomorphism $P_{L}(B) \rightarrow \mathbb{S}^{B}$ is given by the double exponential transpose of $B \stackrel{\left\ulcorner\Delta_{B}\right\urcorner}{\longrightarrow} \mathbb{S}^{B} \stackrel{\diamond_{B}}{\longrightarrow} \mathbb{S}^{P_{L}(B)}$ (see Lemma 4.2.5) and so we are done.

For (iii) note that by the definition of the functor $I d l$ the image of $I d l(h)^{o p}$ : $\operatorname{Idl}\left(A^{o p}\right) \rightarrow \operatorname{Idl}\left(B^{o p}\right)$ is equal to the double exponential transpose of

$$
\operatorname{Idl}\left(A^{o p}\right) \stackrel{i_{A}}{\longrightarrow} \mathbb{S}^{A} \stackrel{\mathbb{S}^{h}}{\longrightarrow} \mathbb{S}^{B}
$$

followed by $\mathbb{S}^{I d l\left(A^{o p}\right)} \stackrel{\varepsilon_{A}}{\longrightarrow} \mathbb{S}^{A}$. I.e. equal to

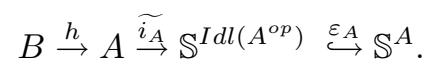

But we've already observed that $\varepsilon_{A} \tilde{i_{A}}=\left\ulcorner\geq_{A}\right\urcorner$ and so this effectively completes the proof since the exponential transpose of $\left\ulcorner\geq_{A}\right\urcorner h$ classifies the open $(h \times$ $\left.I d_{A}\right)^{*}\left(\geq_{A}\right)$.

Notation 4.5.6. We have established a relationship between maps $\operatorname{Idl}\left(A^{o p}\right) \rightarrow$ $\operatorname{Idl}\left(B^{o p}\right)$ of $\mathcal{C}$ and open relations on $B \times A$. We shall follow a notation that if $R$ is such a relation then $n_{R}$ is the corresponding map from $\operatorname{Idl}\left(A^{o p}\right)$ to $\operatorname{Idl}\left(B^{o p}\right)$.

Lemma 4.5.7. With the notation just introduced,

(a) for any $n_{R}: \operatorname{Idl}\left(A^{o p}\right) \rightarrow \operatorname{Idl}\left(B^{o p}\right)$

$$
\begin{array}{ccc}
\mathbb{S}^{I d l\left(B^{o p}\right)} & \stackrel{\mathbb{S}^{n} R}{\longrightarrow} & \mathbb{S}^{I d l\left(A^{o p}\right)} \\
\downarrow \varepsilon_{B} & & \downarrow \varepsilon_{A} \\
\mathbb{S}^{B} & \stackrel{\alpha_{R}}{\longrightarrow} & \mathbb{S}^{A}
\end{array}
$$

commutes; and,

(b) given $n_{R}: \operatorname{Idl}\left(A^{o p}\right) \rightarrow \operatorname{Idl}\left(B^{o p}\right)$ and $n_{R^{\prime}}: \operatorname{Idl}\left(B^{o p}\right) \rightarrow \operatorname{Idl}\left(C^{o p}\right)$

$$
n_{R^{\prime}} n_{R}=n_{R^{\prime} ; R}
$$

Proof. (a) Firstly

$$
\begin{aligned}
\ulcorner R\urcorner & =\varepsilon_{A} \mathbb{S}^{n_{R}} \widetilde{i_{B}} \\
& =\varepsilon_{A} \mathbb{S}^{n_{R}} \delta_{B}\left\ulcorner\Delta_{B}\right\urcorner,
\end{aligned}
$$

the first line by construction of $n_{R}$ and the second since $\delta_{B}\left\ulcorner\Delta_{B}\right\urcorner=\widetilde{i_{B}}$ was established in the previous lemma. Therefore $\varepsilon_{A} \mathbb{S}^{n_{R}} \delta_{B}=\alpha_{R}$ by Lemma 4.2.5. So the result follows since $\delta_{B} \varepsilon_{B}=I d$. 
(b) Under the order isomorphism (i) of the previous lemma the map $C \rightarrow \mathbb{S}^{A}$ corresponding to $n_{R^{\prime}} n_{R}$ is given by the double exponential transpose of

$$
\operatorname{Idl}\left(A^{o p}\right) \stackrel{n_{R}}{\rightarrow} \operatorname{Idl}\left(B^{o p}\right) \stackrel{n_{R^{\prime}}}{\longrightarrow} \operatorname{Idl}\left(C^{o p}\right) \stackrel{i_{C}}{\hookrightarrow} \mathbb{S}^{C}
$$

followed by $\mathbb{S}^{I d l\left(A^{o p}\right)} \stackrel{\varepsilon_{A}}{\hookrightarrow} \mathbb{S}^{A}$. Using party (a) the map $C \rightarrow \mathbb{S}^{A}$ corresponding to $n_{R^{\prime}} n_{R}$ is therefore given by

$$
\begin{aligned}
& C \stackrel{\widetilde{i_{G}}}{\longrightarrow} \mathbb{S}^{I d l\left(C^{o p}\right)} \stackrel{\mathbb{S}^{n} R^{\prime}}{\longrightarrow} \mathbb{S}^{I d l\left(B^{o p}\right)} \\
& \mathbb{S}^{I d l\left(B^{o p}\right)} \stackrel{\varepsilon_{B}}{\longrightarrow} \mathbb{S}^{B} \stackrel{\alpha_{R}}{\longrightarrow} \mathbb{S}^{A}
\end{aligned}
$$

which is equal to $C \stackrel{\left\ulcorner R^{\prime}\right\urcorner}{\longrightarrow} \mathbb{S}^{B} \stackrel{\alpha_{R}}{\longrightarrow} \mathbb{S}^{A}$, i.e. to $\left\ulcorner R^{\prime} ; R\right\urcorner$ as required.

\section{Applications}

\subsection{The fundamental theorem of topos theory}

Before stating and proving the fundamental theorem of topos theory we recall some facts about local homeomorphisms.

Definition 5.1.1. A morphism $f: X \rightarrow Y$ in $\mathcal{C}$ is a local homeomorphism provided it is open and the diagonal $X \rightarrow X \times_{Y} X$ is also open.

When $\mathcal{C}=$ Loc the usual definition for local homeomorphism is that $X$ has an open cover $\left(a_{i}\right)_{i \in I}$ such that each $a_{i} \hookrightarrow X \stackrel{f}{\rightarrow} Y$ is isomorphic to an open of $Y$. However our definition is equivalent (see, for example, Lemma C3.1.15 of [J02]) and is also a good translation of the topological situation since a map between topological spaces is a local homeomorphism if and only if it is open and its diagonal is open.

For any object $X$ in $\mathcal{C}$ we have that $\mathbf{L H} / X=\mathbf{D i s}_{\mathcal{C} / X}$ (see [T04] for the axiomatic proof). We shall also need the following easy extension of this observation:

Lemma 5.1.2. If $A$ is a discrete object of $\mathcal{C}$ then $\operatorname{Dis}_{\mathcal{C} / A}=\left(\operatorname{Dis}_{\mathcal{C}}\right) / A$.

Proof. Since $\mathbf{L H} / A=\operatorname{Dis}_{\mathcal{C} / A}$, we must check two things: (i) for any local homeomorphism $f: Y \rightarrow A, Y$ is necessarily discrete; and, (ii) for any discrete $B$ and for any map $f: B \rightarrow A, f$ is necessarily a local homeomorphism.

For (i), $!^{Y}: Y \stackrel{f}{\rightarrow} A \stackrel{!^{A}}{\rightarrow} 1$ must be open since both $f$ and $!^{A}$ are, the latter by assumption that $A$ is discrete. The inclusion $i: Y \times_{A} Y \hookrightarrow Y \times Y$ is open since it is the pullback (along $Y \times Y \stackrel{f \times f}{\rightarrow} A \times A$ ) of the open diagonal $A \hookrightarrow A \times A$. But then the diagonal $Y \hookrightarrow Y \times Y$ must be open since it is the composition of the open $Y \rightarrow Y \times{ }_{A} Y$ (which is open by assumption that $f$ is a homeomorphism) and $i$. It follows that $Y$ is discrete.

For (ii) firstly $f$ must be open since it is a morphism between discrete objects. But the diagonal $B \rightarrow B \times{ }_{A} B$ is open since it is the pullback (along $i: B \times{ }_{A} B \hookrightarrow$ $B \times B$ ) of the open diapasonal $B \rightarrow B \times B$. This proves that $f$ is a local homeomorphism. 
For an elementary topos $\mathcal{E}, \mathcal{E}$ is of course equivalent to the category of discrete locales over $\mathcal{E}$. In fact the inclusion $\mathcal{E} \hookrightarrow \mathbf{L o c}_{\mathcal{E}}$ has a right adjoint: send any locale $X$ to the set $\operatorname{Loc}_{\mathcal{E}}(1, X)$ (i.e. the set of points of $X$ ). What is remarkable (and originally observed by Paul Taylor as part of his Abstract Stone Duality programme) is that, in the other direction, if the inclusion $\operatorname{Dis}_{\mathcal{C}} \hookrightarrow \mathcal{C}$ has a right adjoint, then $\mathbf{D i s}_{\mathcal{C}}$ is a topos.

Proposition 5.1.3. If the inclusion $\operatorname{Dis}_{\mathcal{C}} \hookrightarrow \mathcal{C}$ has a right adjoint then $\operatorname{Dis}_{\mathcal{C}}$ is a topos.

We will use the notation $\left({ }_{-}\right)_{d}: \mathcal{C} \rightarrow \mathbf{D i s}_{\mathcal{C}}$ for such a right adjoint if it exists.

Proof. A category is a topos provided it is cartesian and has power objects. We have commented already that $\mathbf{D i s}_{\mathcal{C}}$ is cartesian and so we are left checking that $\operatorname{Dis}_{\mathcal{C}}$ has power objects. For any discrete object $A$, let $P A$ denote the object $\left(\mathbb{S}^{A}\right)_{d}$ of $\mathbf{D i s} \mathcal{C}_{\mathcal{C}}$. To prove that this defines a power object of $A$ we must find a monomorphism $\in_{A} \hookrightarrow P A \times A$ such that for any monomorphism $R \hookrightarrow B \times A$ there exists a unique map $r: B \rightarrow P A$ such $R$ is the pullback of $\in_{A}$ along $r \times I d_{A}$. Now all morphisms in $\mathbf{D i s}_{\mathcal{C}}$ are open and all monomorphisms in $\operatorname{Dis}_{\mathcal{C}}$ are regular. The open regular monomorphisms in $\mathcal{C}$ are exactly the open subobjects, and so $\operatorname{Sub}_{\text {Dis }_{\mathcal{C}}}(B \times A) \cong \mathcal{C}(B \times A, \mathbb{S})$. So we have that

$$
\begin{aligned}
\operatorname{Dis}_{\mathcal{C}}(B, P A) & \cong \mathcal{C}\left(B, \mathbb{S}^{A}\right) \\
& \cong \mathcal{C}(B \times A, \mathbb{S}) \\
& \cong \operatorname{Sub}_{\text {Dis }_{\mathcal{C}}}(B \times A) .
\end{aligned}
$$

This bijection is natural in $B$, so if $\epsilon_{A}$ is defined as the mate of $I d_{P A}$ then any monomorphism $R \hookrightarrow B \times A$ is the pullback of $\in_{A}$ along $r \times I d_{A}$, where $r: B \rightarrow P A$ is the mate of $R$ under the bijection.

The fundamental theorem of topos theory now becomes a categorical triviality:

Theorem 5.1.4. If $\mathcal{E}$ is an elementary topos and $A$ is an object of $\mathcal{E}$ then $\mathcal{E} / A$ is an elementary topos.

Proof. Firstly, as remarked before the last proposition, as $\mathcal{E}$ is a topos, $\mathcal{E} \hookrightarrow$ $\mathbf{L o c}_{\mathcal{E}}$ has a right adjoint. Now $\operatorname{Dis}_{\mathbf{L o c} \mathcal{E} / A} \simeq \mathcal{E} / A$ by the lemma and so it is sufficient to find a right adjoint to the inclusion $\mathcal{E} / A \hookrightarrow \operatorname{Loc}_{\mathcal{E}} / A$. Define $\left({ }_{-}\right)_{d_{A}}: \mathbf{L o c}_{\mathcal{E}} / A \rightarrow \mathcal{E} / A$ by sending an object $f: X \rightarrow A$ of $\operatorname{Loc}_{\mathcal{E}} / A$ to $(f)_{d}:$ $X_{d} \rightarrow A_{d} \cong A$. It is easy to verify that $\left({ }_{-}\right)_{d_{A}}$ is the required right adjoint.

For the next section we will need the following basic lemma which is showing that if $\left({ }_{-}\right)_{d}$ exists right adjoint to the inclusion $i: \mathbf{D i s}_{\mathcal{C}} \hookrightarrow \mathcal{C}$ then the induced adjoint transpose can commute with exponential transpose (for functions to power sets at least). Recall, of course, that a power set $P A$ can be written as the exponential $\Omega^{A}$ where $\Omega$ is the subobject classifier (i.e. $P 1$ ). For toposes we use the standard notation $\chi_{I}: A \rightarrow \Omega$ for the map that classifies a monomorphism $I \hookrightarrow A$. This is in contrast to our notation for $\mathcal{C}$ where we have not distinguished notationally between $a \hookrightarrow X$, an open regular monomorphism, and $a: X \rightarrow \mathbb{S}$. 
Lemma 5.1.5. If $i: \mathbf{D i s}_{\mathcal{C}} \hookrightarrow \mathcal{C}$ has a right adjoint then

(i) for any objects $Y$ of $\mathcal{C}$ and $A$ of $\operatorname{Dis}_{\mathcal{C}}, Y_{d}^{A} \cong\left(Y^{A}\right)_{d}$,

(ii) if $R \hookrightarrow C \times B$ is a monomorphism in Dis ${ }_{\mathcal{C}}$ then the mate of $C \stackrel{\left\ulcorner\chi_{R}\right\urcorner}{\longrightarrow}$ $\mathbb{S}_{d}^{B} \cong\left(\mathbb{S}^{B}\right)_{d}$ under the adjunction $i \dashv\left(\left(_{-}\right)_{d}\right.$ is $C \stackrel{\ulcorner R\urcorner}{\longrightarrow} \mathbb{S}^{B}$ where $R: C \times B \rightarrow \mathbb{S}$ classifies the open $R$; and,

(iii) the conunit at $\mathbb{S}$ of the adjunction $i \dashv\left({ }_{-}\right)_{d}$ is the map $T:(\mathbb{S})_{d} \rightarrow \mathbb{S}$ that classifies the open 'true', i.e. the top element of the subobject classifier $(\mathbb{S})_{d}$.

Proof. (i) This is a routine diagram chase given that finite products of Dis $\mathbf{S}_{\mathcal{C}}$ are created in $\mathcal{C}$.

(ii) The mate of $C \stackrel{\ulcorner R\urcorner}{\longrightarrow} \mathbb{S}^{B}$ under the adjunction is $C \stackrel{\ulcorner R\urcorner_{d}}{\longrightarrow}\left(\mathbb{S}^{B}\right)_{d}$ (passing through the isomorphism $C \cong C_{d}$ without notation). Under the isomorphism of (i) the exponential transpose of $C \stackrel{\ulcorner R\urcorner_{d}}{\longrightarrow}\left(\mathbb{S}^{B}\right)_{d} \cong \mathbb{S}_{d}^{B}$ is $C \times B \stackrel{R_{d}}{\longrightarrow} \mathbb{S}_{d}$. But $(-)_{d}$ preserves the pullback

$$
\begin{array}{ccc}
R & \rightarrow & 1 \\
\downarrow & & \downarrow 1_{\mathbb{S}} \\
C \times B & \stackrel{R}{\rightarrow} & \mathbb{S}
\end{array}
$$

and so $R_{d}=\chi_{R}$ giving $\ulcorner R\urcorner_{d}=\left\ulcorner\chi_{R}\right\urcorner$ as required.

(iii) is immediate from (ii) since the counit is the mate of identity.

\subsection{Localic slice stability}

We now embark on a proof that for any locale $Y$,

$$
\operatorname{Loc}_{S h(Y)} \simeq \operatorname{Loc} / Y .
$$

This result is of course well known, but its usual proof does require some understanding of constructions internal in a sheaf topos. Although we appear to be able to dispense with most of the topos theory, it does not appear possible to entirely dispense with sheaf theory as the following well known observations are required:

Lemma 5.2.1. For any locale $Y$,

(a) $\operatorname{Sh}(Y) \simeq \mathbf{L H} / Y$,

(b) the inclusion $\mathbf{L H} / Y \hookrightarrow \mathbf{L o c} / Y$ has a right adjoint. This right adjoint $\left({ }_{-}\right)_{d_{Y}}$ sends any $X_{f}$ to the sheaf of sections, i.e. defined by

$$
\left(X_{f}\right)_{d_{Y}}(a)=\{s: a \rightarrow X \mid f s=a \hookrightarrow Y\}
$$

for any open $a \hookrightarrow Y$ of $Y$; and,

(c) if $f: X \rightarrow Y$ is a map of locales then the pullback functor $f^{*}: \mathbf{L H} / Y \rightarrow$ $\mathbf{L H} / X$ is cartesian and has a right adjoint, denoted $f_{*}$ say. As an action on sheaves, $f_{*}(F)(a)=F \Omega f(a)$.

In part (c) we are following a notation whereby we use $\Omega X$ for the frame corresponding to a locale $X$ and are using $\Omega f: \Omega Y \rightarrow \Omega X$ for the frame homomorphism corresponding to a locale map $f: X \rightarrow Y$. 
Proof. Consult, for example, Ch. VI of [J82]. In (c), the right adjoint is the direct image of the geometric morphism $f: S h(X) \rightarrow S h(Y)$.

Note that for any locale map $f: X \rightarrow Y$ the adjunction $f^{*} \dashv f_{*}: \mathbf{L H} / X \leftrightarrows$ $\mathbf{L H} / Y$ gives rise to an adjunction $f^{*} \dashv f_{*}: \wedge-\mathbf{S L a t}_{\mathbf{L H} / X} \leftrightarrows \wedge-\mathbf{S L a t}_{\mathbf{L H} / Y}$ since both $f_{*}$ and $f^{*}$ are cartesian and so preserve the relevant structure. Further note that, by exactly the same reasoning, in the situations where $i:$ Dis $_{\mathcal{C}} \hookrightarrow \mathcal{C}$ has a right adjoint $\left({ }_{-}\right)_{d}$, since $i$ preserves finite products, there is an induced adjunction: $\wedge-$ SLat $_{\mathbf{D i}} \mathbf{s}_{\mathcal{C}} \leftrightarrows \wedge-$ SLat $_{\mathcal{C}}$

Part (b) tells us:

Proposition 5.2.2. For any locale $Y, \mathbf{L H} / Y$ is a topos.

Proof. This is immediate from 5.1.3 since $\mathbf{L H} / Y=\mathbf{D i s}_{\mathbf{L o c} / Y}$.

Say $\mathbb{D} \equiv(\mathcal{D}, I d \stackrel{\downarrow}{\longrightarrow} \mathcal{D}, \mathcal{D} \mathcal{D} \stackrel{\cup}{\longrightarrow} \mathcal{D})$ denotes the lower closed power set monad on $\wedge-\mathbf{S L a t}_{\mathbf{L H} / Y}$. So, relative to the topos $\mathbf{L H} / Y$, for any internal semilattice $A, \mathcal{D}(A)$ is the set of lower closed subsets of $A$. A frame is exactly an algebra of $\mathbb{D}$ and a $\mathbb{D}$-algebra homomorphism is exactly a frame homomorphism. Our aim of proving

$$
\operatorname{Loc}_{S h(Y)} \simeq \operatorname{Loc} / Y .
$$

therefore reduces to proving that

$$
\wedge-\mathbf{S L a t}_{\mathbf{L H} / Y}^{\mathbb{D}} \simeq \mathbf{L o c}^{o p} / Y .
$$

We do this in two stages. Firstly we show that the functor $I d l_{Y}\left({ }_{-}\right)^{o p}: \wedge-$ $\mathbf{S L a t}_{\mathbf{L H} / Y} \rightarrow \mathbf{L o c}^{o p} / Y$ has a monadic right adjoint (say denoted $U_{Y}$ ). Secondly we check that the induced monad, $U_{Y} I d l_{Y}\left({ }_{-}\right)^{o p}$, is isomorphic to $\mathbb{D}$.

Proposition 5.2.3. For any locale $Y, I d l_{Y}\left({ }_{-}\right)^{o p}: \wedge-\mathbf{S L a t}_{\mathbf{L H} / Y} \rightarrow \mathbf{L o c}^{o p} / Y$ has a monadic right adjoint.

Proof. If $X_{f}$ is an object of Loc $/ Y$ define $U_{Y}\left(X_{f}\right)$ to be $f_{*}\left(\left(\mathbb{S}_{X}\right)_{d_{X}}\right)$. If $h$ : $X_{f^{\prime}}^{\prime} \rightarrow X_{f}$ is a morphism of Loc/Y then let $U_{Y}(h): f_{*}\left(\left(\mathbb{S}_{X}\right)_{d_{X}}\right) \rightarrow f_{*}^{\prime}\left(\left(\mathbb{S}_{X^{\prime}}\right)_{d_{X^{\prime}}}\right)$ be the map $f_{*}\left(\widetilde{\chi_{h^{*} \top}}\right)$ where $\widetilde{(-)}$ is adjoint transpose via $h^{*} \dashv h_{*}$. The map $\top: 1_{X^{\prime}} \rightarrow\left(\mathbb{S}_{X^{\prime}}\right)_{d_{X^{\prime}}}$ is the universal monomorphism in $\mathbf{L H} / X^{\prime}$ which exists since we have established that this category is a topos. The pullback (along $h$ ) of this monomorphism is a monomorphisms and is therefore classified by a map to $\left(\mathbb{S}_{X}\right)_{d_{X}}$ and we are defining $U_{Y}(h)$ to be the $f_{*}$ applied to the adjoint transpose of this classifying map.

We now check that this provides a right adjoint to $I d l_{Y}\left({ }_{-}\right)^{o p}$. For any object $A_{g}$ of $\wedge-\mathbf{S L a t}_{\mathbf{L H} / Y}$, we have that

$$
\begin{aligned}
\wedge-\operatorname{SLat}_{\mathbf{L H} / Y}\left(A_{g}, f_{*}\left(\left(\mathbb{S}_{X}\right)_{d_{X}}\right)\right) & \cong \wedge-\mathbf{S L a t}_{\mathbf{L H} / X}\left(f^{*} A_{g},\left(\mathbb{S}_{X}\right)_{d_{X}}\right) \\
& \cong \wedge-\operatorname{SLat}_{\mathbf{L o c} / X}\left(f^{*} A_{g}, \mathbb{S}_{X}\right)
\end{aligned}
$$


On the other hand

$$
\begin{aligned}
(\operatorname{Loc} / Y)^{o p}\left(I d l_{Y}\left(A_{g}\right)^{o p}, X_{f}\right) & =(\mathbf{L o c} / Y)\left(X_{f}, I d l_{Y}\left(A_{g}^{o p}\right)\right) \\
& \cong(\mathbf{L o c} / X)\left(1, I d l_{X}\left(f^{*} A_{g}^{o p}\right)\right)
\end{aligned}
$$

since the $I d l$ construction is stable under change of base. This verifies that $I d l_{Y}()^{o p} \dashv U_{Y}\left(X_{f}\right)$ since by construction the points of $I d l_{X}\left(f^{*} A_{g}^{o p}\right)$ are exactly the meet semilattice homomorphisms from $f^{*} A_{g}$ to $\mathbb{S}_{Y}$. The naturality of the order isomorphisms involved is routine from our definition of $U_{Y}$ on morphisms since (lemma 5.1.5) the counit of the coreflection $\left({ }_{-}\right)_{d}$ classifies the universal monomorphism $T$.

For completeness we now check this naturality with respect to $X_{f}$. Say $t: A_{g} \rightarrow U_{Y}\left(X_{f}\right)$ and $\widehat{t}: X_{f} \rightarrow I d l_{Y}\left(A_{g}^{o p}\right)$ is its mate. We are given $h: X_{f^{\prime}}^{\prime} \rightarrow$ $X_{f}$. Under the order isomorphisms between the homsets we have the following mappings:

$$
\begin{aligned}
& A_{g} \stackrel{t}{\rightarrow} U_{Y}\left(X_{f}\right) \stackrel{U_{Y}(h)}{\longrightarrow} U_{Y}\left(X_{f^{\prime}}^{\prime}\right) \\
& A_{g} \stackrel{t}{\rightarrow} f_{*}\left(\left(\mathbb{S}_{X}\right)_{d_{X}}\right) \stackrel{f_{*}\left(\widetilde{\chi_{h^{*} T}}\right)}{\longrightarrow} f_{*}^{\prime}\left(\left(\mathbb{S}_{X^{\prime}}\right)_{d_{X^{\prime}}}\right) \\
& h^{*}\left(f^{*} A_{g}\right) \stackrel{h^{*}(\bar{t})}{\rightarrow} h^{*}\left(\left(\mathbb{S}_{X}\right)_{d_{X}}\right) \stackrel{\chi_{h^{*} T}}{\longrightarrow}\left(\mathbb{S}_{X^{\prime}}\right)_{d_{X^{\prime}}} \quad \bar{t} \text { mate of } t \text { via } f^{*}-! f_{*} \\
& h^{*}\left(f^{*} A_{g}\right) \stackrel{h^{*}(\bar{t})}{\rightarrow} h^{*}\left(\left(\mathbb{S}_{X}\right)_{d_{X}}\right) \stackrel{\chi_{h^{*}}}{\longrightarrow}\left(\mathbb{S}_{X^{\prime}}\right)_{d_{X^{\prime}}} \stackrel{T^{\prime}}{\rightarrow} \mathbb{S}_{X^{\prime}}
\end{aligned}
$$

where the last line is because $T^{\prime}$ is the conunit of the adjunction $i_{X^{\prime}} \dashv d_{X^{\prime}}$, see (iii) of 5.1.5. But $h^{*}\left(\left(\mathbb{S}_{X}\right)_{d_{X}}\right) \stackrel{\chi_{h^{*}}}{\longrightarrow}\left(\mathbb{S}_{X^{\prime}}\right)_{d_{X^{\prime}}} \stackrel{\mathrm{T}^{\prime}}{\rightarrow} \mathbb{S}_{X^{\prime}}$ is equal to $h^{*} T$ since this is the open that it classifies and so we have that $U_{Y}(h) t$ maps to $h^{*}$ applied to

$$
f^{*} A_{g} \stackrel{\bar{t}}{\rightarrow}\left(\mathbb{S}_{X}\right)_{d_{X}} \stackrel{\top}{\rightarrow} \mathbb{S}_{X}
$$

On the other hand $X_{f^{\prime}}^{\prime} \stackrel{h}{\rightarrow} X_{f} \stackrel{\hat{t}}{\rightarrow} I d l_{Y}\left(A_{g}^{o p}\right)$ maps to the meet semilattice homomorphisms

$$
\begin{array}{ll}
A_{g} \rightarrow \mathbb{S}_{Y}^{X_{f}} \stackrel{\mathbb{S}_{Y}^{h}}{\longrightarrow} \mathbb{S}_{Y}^{X_{f^{\prime}}^{\prime}} & \\
f^{*} A_{g} \stackrel{T \bar{t}}{\rightarrow} \mathbb{S}_{X} \stackrel{\mathbb{S}_{X}^{h}}{\longrightarrow} \mathbb{S}_{X}^{X_{h}^{\prime}} & \quad \text { change base to } X \\
h^{*} f^{*} A_{g} \stackrel{h^{*}(T \bar{t})}{\longrightarrow} h^{*}\left(\mathbb{S}_{X}\right) & \text { change base to } X^{\prime} .
\end{array}
$$

This completes our check of naturality.

To verify that is $U_{Y}$ monadic we check that (i) it has a left adjoint (clearly done already), (ii) it reflects isomorphisms and (iii) $(\text { Loc } / Y)^{o p}$ has and $U_{Y}$ preserves coequalizers of $U_{Y}$-split pairs (i.e. pairs $h_{1}, h_{2}: X_{f} \rightarrow Z_{g}$ such that $U_{Y}\left(h_{1}\right), U_{Y}\left(h_{2}\right)$ are part of a split coequalizer diagram in $\wedge-\mathbf{S L a t}_{\mathbf{L H} / Y}$.)

To prove (ii) and (iii) we use an explicit description of the sheaf corresponding to $U_{Y}\left(X_{f}\right)$ and of the morphisms in $S h(Y)$ corresponding to $U_{Y}(h)$. Since 
$\left({ }^{-} d_{X}\right.$ is the functor that takes the sheaf of sections, as a sheaf we have that $\left(\mathbb{S}_{X}\right)_{d_{X}}(a) \cong \downarrow a$ for every open $a \in \Omega X$. Therefore by the description of $f_{*}$ given in part (c) of the lemma we have that the sheaf corresponding to $U_{Y}\left(X_{f}\right)$ is

$$
U_{Y}\left(X_{f}\right)(b) \cong \downarrow \Omega f(b)
$$

for every $b \in \Omega Y$.

As for $U_{Y}(h)$, note first that under the order isomorphism just established, for any $c \leq \Omega f(b)$, the point of $f_{*}\left(\mathbb{S}_{X}\right)_{d_{X}}$ corresponding to $c$ is the adjoint transpose (under $f^{*} \dashv f_{*}$ ) of $\chi_{c}: f^{*} b \rightarrow\left(\mathbb{S}_{X}\right)_{d_{X}}$. The image of $c$ under $U_{Y}(h)$ is therefore the adjoint transpose (under $\left.\left(f^{\prime}\right)^{*} \dashv f_{*}^{\prime}\right)$ of

$$
b \stackrel{\overline{\chi_{c}}}{\longrightarrow} f_{*}\left(\left(\mathbb{S}_{X}\right)_{d_{X}}\right) \stackrel{\left.f_{*} \stackrel{\left(\widetilde{\chi_{h^{*} \top}}\right.}{\longrightarrow}\right)}{\prime} f_{*}^{\prime}\left(\left(\mathbb{S}_{X^{\prime}}\right)_{d_{X^{\prime}}}\right)
$$

which is $h^{*}(c)$. In other words, as a natural transformation (i.e. as a morphisms in $S h(Y)), U_{Y}(h)(c)=\Omega h(c)$ for any $c \in U_{Y}\left(X_{f}\right)(b)$. Note that it is then immediate, by considering $b=1$, that $U_{Y}$ is conservative and so (ii) is checked.

For (iii) certainly $(\text { Loc } / Y)^{o p}$ has such coequalizers as Loc is cartesian. Say $E_{e} \stackrel{i}{\hookrightarrow} X_{f} \underset{h_{2}}{\stackrel{h_{1}}{\longrightarrow}} Z_{g}$ is an equalizer diagram in Loc/ $Y$ such that there is a split coequalizer

$$
U_{Y}\left(Z_{g}\right) \underset{U_{Y}\left(h_{2}\right)}{\stackrel{U_{Y}\left(h_{1}\right)}{\longrightarrow}} U_{Y}\left(X_{f}\right) \stackrel{\gamma}{\rightarrow} F
$$

in $S h(Y)$. We must check that $F \cong U_{Y}\left(E_{e}\right)$. Firstly note that for any open $b \hookrightarrow Y$,

$$
\downarrow \Omega g(b) \stackrel{\Omega h_{1}}{\longrightarrow} \downarrow \Omega f(b) \stackrel{\Omega i}{\longrightarrow} \downarrow \Omega e(b)
$$

is a frame coequalizer since the pullback of the equalizer diagram $E \stackrel{i}{\hookrightarrow} X \underset{h_{2}}{\stackrel{h_{1}}{\longrightarrow}} Z$ along the open $\Omega g(b) \hookrightarrow Z$ is again an equalizer diagram in Loc. Now, for every open $b$ of $Y$ we have that

$$
U_{Y}\left(Z_{g}\right)(b) \underset{U_{Y}\left(h_{2}\right)_{b}}{\stackrel{U_{Y}\left(h_{1}\right)_{b}}{\longrightarrow}} U_{Y}\left(X_{f}\right)(b) \stackrel{\gamma_{b}}{\rightarrow} F(b)
$$

is a split coequalizer diagram in $\wedge$ - SLat and since the category of frames is monadic over $\wedge-$ SLat we have that $[*]$ is a coequalizer in the category of frames. But

$$
U_{Y}\left(Z_{g}\right)(b) \underset{U_{Y}\left(h_{2}\right)_{b}}{\stackrel{U_{Y}\left(h_{1}\right)_{b}}{\longrightarrow}} U_{Y}\left(X_{f}\right)(b)
$$


is isomorphic to

$$
\downarrow \Omega g(b) \underset{\Omega h_{2}}{\stackrel{\Omega h_{1}}{\longrightarrow}} \downarrow \Omega f(b)
$$

and so $F(b) \cong \downarrow \Omega e(b) \cong U_{Y}\left(E_{e}\right)(b)$ for every $b$. Hence $F \cong U_{Y}\left(E_{e}\right)$ as required. This completes the proof of (iii) and so shows that $U_{Y}$ is monadic as required.

Clearly we want the monad defined in the proposition to be equivalent to $\mathbb{D}$ on $\wedge-\mathbf{S L a t}_{\mathbf{L H} / X}$. In fact we know that we have the correct monad even without going to the slice:

Proposition 5.2.4. If $\operatorname{Dis}_{\mathcal{C}} \hookrightarrow \mathcal{C}$ has a right adjoint and Idl(_- $)^{o p}: \wedge-$ $\mathbf{S L a t}_{\text {Dis }} \rightarrow \mathcal{C}^{o p}$ has a right adjoint (say denoted $U$ ), then the monad induced on $\wedge-\mathbf{S L a t}_{\text {Dis }}$ is (naturally isomorphic to) the lower powerset monad $\mathbb{D}$ on $\wedge-$ SLat $_{\text {Disc }}$.

Proof. Firstly we check that for all meet semilattices $A$ and $B$

$$
\wedge-\operatorname{SLat}_{\mathbf{D i s}}\left(A, U \operatorname{Idl}\left(B^{o p}\right)\right) \cong \wedge-\operatorname{SLat}_{\mathbf{D i s}_{\mathcal{C}}}(A, \mathcal{D} B) .
$$

Note that $\mathcal{D} B$ is the split of $P B \underset{\downarrow}{\stackrel{I d}{\longrightarrow}} P B$ where, of course, $P B=\left(\mathbb{S}^{B}\right)_{d}$. Since $\mathcal{D} B \hookrightarrow P B$ is a meet semilattice homomorphism it is easy to check that

$$
\wedge-\operatorname{SLat}_{\text {Dis }_{\mathcal{C}}}(A, \mathcal{D} B) \cong\{f: A \rightarrow P B \mid f \wedge-S \text { Lat hom. and } \downarrow f=f\} .
$$

Now $\wedge-\operatorname{SLat}_{\operatorname{Dis}_{\mathcal{C}}}\left(A, \operatorname{UIdl}\left(B^{o p}\right)\right) \cong\left\{n: A \rightarrow \mathbb{S}^{B} \mid n \wedge-\right.$ SLat hom. and $\left.\alpha_{\geq_{B}} n=n\right\}$ by Lemma 4.5.5. So to complete a proof $(*)$ we must but check that

$$
\downarrow=\left(\alpha_{\geq_{B}}\right)_{d}
$$

since we have commented already that the adjunction $i \dashv\left(\left(_{-}\right)_{d}: \mathbf{D i s}_{\mathcal{C}} \longleftarrow \mathcal{C}\right.$ gives rise to an adjunction $\wedge-$ SLat $_{\text {Dis }} \longleftarrow \wedge-$ SLat $_{\mathcal{C}}$.

For (a) it is sufficient to show that for any monomorphism $R \hookrightarrow C \times B$ of $\operatorname{Dis}_{\mathcal{C}}$ that $\downarrow\left\ulcorner\chi_{R}\right\urcorner=\left(\alpha_{\geq_{B}}\right)_{d}\left\ulcorner\chi_{R}\right\urcorner$. But by Lemma 5.1.5 the mate of $\left(\alpha_{\geq_{B}}\right)_{d}\left\ulcorner\chi_{R}\right\urcorner$ under the adjunction $i \dashv()_{d}$ is

$$
\begin{aligned}
& C \stackrel{\ulcorner R\urcorner}{\longrightarrow} \mathbb{S}^{B} \stackrel{\alpha_{\geq_{B}}}{\longrightarrow} \mathbb{S}^{B} \\
& =C \stackrel{\left\ulcorner R ; \geq_{B}\right\urcorner}{\longrightarrow} \mathbb{S}^{B}
\end{aligned}
$$

Now $C \stackrel{\left\ulcorner\chi_{R}\right\urcorner}{\longrightarrow} P B \stackrel{\downarrow}{\longrightarrow} P B$ is equal to $C \stackrel{\left\ulcorner\chi_{R_{i} \geq_{B}}^{\longrightarrow}\right\urcorner}{\longrightarrow} P B$ since relational composition is calculated in $\mathbf{D i s}_{\mathcal{C}}$ and so uses the ; formulae developed. Therefore $\downarrow\left\ulcorner\chi_{R}\right\urcorner=\left(\alpha_{\geq_{B}}\right)_{d}\left\ulcorner\chi_{R}\right\urcorner$ follows by another application of Lemma 5.1.5 and so 
(a) is established since we have argued with arbitrary $R$. Therefore we have established (*) and so have established a natural isomorphism $\phi: \mathcal{D} \rightarrow U \operatorname{Idl}\left({ }_{-}\right)^{o p}$.

The remainder of the proof checks that the monad structure induced by the adjunction $I d l\left(_{-}\right)^{o p} \dashv U$ is naturally isomorphic to $\mathbb{D}$. Note that by construction $\phi_{B}$ is the adjoint transpose of the opposite of a map $\operatorname{Idl}\left(B^{o p}\right) \rightarrow \operatorname{Idl}\left((\mathcal{D} B)^{o p}\right)$ which itself corresponds to the membership relation $\in_{B} \hookrightarrow \mathcal{D} B \times B$. In other

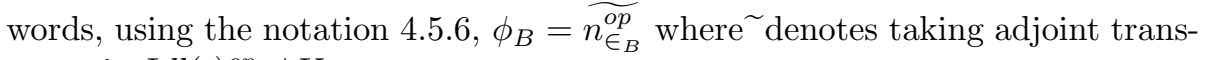
pose via $\operatorname{Idl}\left({ }_{-}\right)^{o p} \dashv U$.

The unit of the monad induced by $\operatorname{Idl}\left({ }_{-}\right)^{o p} \dashv U$, evaluated at $B$, is given by taking the adjoint transpose of the identity on $\operatorname{Idl}\left(B^{o p}\right)$. Given Lemma 4.5.5 we known that this corresponds to $B \stackrel{\ulcorner\geq B\urcorner}{\longrightarrow} \mathbb{S}^{B}$ and so to $\downarrow: B \rightarrow P B$ (lemma 5.1.5 since $\left.\downarrow=\left\ulcorner\chi_{\geq_{B}}\right\urcorner\right)$. Therefore the unit of the monad induced by $I d l\left({ }_{-}\right)^{o p} \dashv U$ maps to the unit of $\mathbb{D} \equiv(\mathcal{D}, I d \stackrel{\downarrow}{\longrightarrow} \mathcal{D}, \mathcal{D D} \stackrel{\cup}{\longrightarrow} \mathcal{D})$ via $(*)$.

To complete the proof we must check that the union operation $\mathcal{D D} \stackrel{\cup}{\longrightarrow} \mathcal{D}$ maps to $U \varepsilon_{I d l\left(\text { I }^{o p} \text { via }\right.}$ via natural isomorphism $\phi$, where $\varepsilon$ is the counit of the adjunction $\operatorname{Idl}\left(_{-}\right)^{o p} \dashv U$. In other words we must verifying that the diagram

$$
\begin{array}{ccc}
\mathcal{D D} B & \operatorname{UIdl}\left(\phi_{B}\right) \phi_{\mathcal{D} B} & \operatorname{UIdl}\left(U \operatorname{UIdl}\left(B^{o p}\right)\right)^{o p} \\
\cup \downarrow & & \downarrow U \varepsilon_{\operatorname{Idl}\left(B^{o p}\right)} \\
\mathcal{D} B & \stackrel{\phi_{B}}{\longrightarrow} & U \operatorname{Idl}\left(B^{o p}\right)
\end{array}
$$

commutes. By taking adjoint transpose this amounts to checking that the diagram

$$
\begin{aligned}
& \operatorname{Idl}\left(\mathcal{D D D} B^{o p}\right) \stackrel{\stackrel{n^{o p}}{\stackrel{\epsilon_{\mathcal{D} B}}{\longrightarrow}}}{\longrightarrow} \operatorname{Idl}\left(\mathcal{D} B^{o p}\right) \stackrel{\operatorname{Idl}\left(\phi_{B}\right)}{\longrightarrow} \operatorname{Idl}\left(\operatorname{UIdl}\left(B^{o p}\right)\right)^{o p} \\
& I d l(\cup) \downarrow \quad \downarrow \varepsilon_{I d l\left(B^{o p}\right)} \\
& \operatorname{Idl}\left(\mathcal{D} B^{o p}\right) \quad \stackrel{n_{\epsilon_{B}}^{o p}}{\longrightarrow} \quad \operatorname{Idl}\left(B^{o p}\right)
\end{aligned}
$$

commutes in $\mathcal{C}^{o p}$. Now $\varepsilon_{I d l\left(B^{o p}\right)}$ is the adjoint transpose of the identity $I d_{U I d l\left(B^{o p}\right)}$ and since this identity factors as

$$
\operatorname{UIdl}\left(B^{o p}\right) \stackrel{\phi_{B}^{-1}}{\longrightarrow} \mathcal{D} B \stackrel{\phi_{B}}{\longrightarrow} U \operatorname{Idl}\left(B^{o p}\right)
$$

(and $\phi_{B}=\widetilde{n_{\epsilon_{B}}^{o p}}$ ) we get that $\varepsilon_{I d l\left(B^{o p}\right)}=n_{\epsilon_{B}}^{o p} \operatorname{Idl}\left(\phi_{B}^{-1}\right)$. So we are left checking that $n_{\in_{\mathcal{D} B}} n_{\epsilon_{B}}=\operatorname{Idl}(\cup)^{o p} n_{\in_{B}}$. By Lemma 4.5.7 $n_{\in_{\mathcal{D} B}} n_{\epsilon_{B}}=n_{\in_{\mathcal{D} B} ; \epsilon_{B}}$ and by (iii) of Lemma 5.1.5 we get that the relation on $\mathcal{D D} B \times \mathcal{D} B$ corresponding to $\operatorname{Idl}(\cup)^{o p}$ is (isomorphic to) the pullback of $\supseteq_{\mathcal{D} B} \hookrightarrow \mathcal{D} B \times \mathcal{D} B$ along $\cup \times I d_{\mathcal{D} B}$. Since it is trivial to check that

$$
\epsilon_{\mathcal{D} B} ; \epsilon_{B}=\left[\left(\cup \times I d_{\mathcal{D} B}\right)^{*} \supseteq_{\mathcal{D} B}\right] ; \epsilon_{B}
$$

we are done.

In summary we have now proved:

Theorem 5.2.5. For any locale $Y, \operatorname{Loc}_{S h(Y)} \simeq \operatorname{Loc} / Y$. 


\section{Concluding comments}

It should be clear that the categorical axioms offered here are not as elegant as could be hoped for. There is no claim in this work that the axioms represent the best way of developing locale theory based purely on categorical assumptions. For example, the interdependencies between the axioms have not been explored and so some axioms may by redundant. The point of the work is rather that (a) the axioms 'do the job' in the sense that they allow us to recover well known constructions and results, (b) the theory is order dual and so simultaneously covers both the theory of proper maps and the theory of open maps and (c) the axioms are slice stable. This paper has focused on (c) and we have covered the following aspects,

(i) the axiomatic framework is slice stable,

(ii) the fundamental theorem of topos theory can be recovered; and

(iii) the logical slice stability of locales can be proved using only some basic assumptions from sheaf theory.

Along the way we have offered a new categorical proof of Vicker's result that $P_{L}(A) \cong \mathbb{S}^{A}$ for discrete $A$ and have developed, axiomatically, some well known properties of the ideal completion locale construction. We end with a table of the axioms together with some technical comments on them. 


\begin{tabular}{|c|c|c|}
\hline Axiom & Description & Comment \\
\hline 1 & $\begin{array}{ll}\text { Order } & \text { enriched } \\
\text { cartesian } & \end{array}$ & \\
\hline 2 & Stable distributive & Necessity of slice stable phrasing open \\
\hline 3 & Sierpiński exists & Not canonical \\
\hline 4 & $\begin{array}{l}\text { Equalizers give rise } \\
\text { to coequalizers in } \\
\text { presheaves over } \mathcal{C}\end{array}$ & $\begin{array}{l}\text { Axiomatic version of the double cover- } \\
\text { age theorem. Equivalent to } \mathbb{P} \text { preserving } \\
\text { coreflexive equalizers in the presence of } \\
\text { Axioms } 7 \text { and } 9\end{array}$ \\
\hline 5 & $\begin{array}{l}\mathbb{S}^{(-)} \text {reflects isomor- } \\
\text { phisms }\end{array}$ & \\
\hline $5^{\prime}$ & $\begin{array}{l}\text { Distributive lattice } \\
\text { homs. } \mathbb{S}^{Y} \rightarrow \mathbb{S}^{X} \\
\text { are of the form } \mathbb{S}^{f} \\
\text { for unique } f: X \rightarrow \\
Y\end{array}$ & Strengthening of Axiom 5 \\
\hline 6 & $\begin{array}{l}\mathbb{S}^{(-)} \text {takes regular } \\
\text { epimorphisms to } \\
\text { monomorphisms }\end{array}$ & $\begin{array}{l}\text { In locale theory } \mathbb{S}^{q} \text { is a regular } \\
\text { monomorphism for regular epi. } q \text { but } \\
\text { this strengthening is not required in ap- } \\
\text { plication. }\end{array}$ \\
\hline 7 & $\begin{array}{l}\text { Double exponentia- } \\
\text { tion over } \mathbb{S} \text { exists }\end{array}$ & $\begin{array}{l}\text { Existence necessary for the other two } \\
\text { power object and the ideal completion } \\
\text { constructions }\end{array}$ \\
\hline 8 & $\begin{array}{l}\text { Power monads are } \\
\mathrm{KZ} / \text { coKZ }\end{array}$ & $\begin{array}{l}\text { Implied by Cauchy completeness of } \\
\text { Kleisli categories. Implies that idem- } \\
\text { potent inflationary/deflationary Kleisli } \\
\text { morphisms split. This splitting (on the } \\
\text { lower side of the duality) gives ideal } \\
\text { completion. }\end{array}$ \\
\hline 9 & $\begin{array}{l}\text { Product is given by } \\
\text { tensor }\end{array}$ & $\begin{array}{l}\text { Can be used to prove pullback stability } \\
\text { results. Open whether necessary as an } \\
\text { additional axiom. }\end{array}$ \\
\hline
\end{tabular}

\section{Appendix}

\subsection{Product as join semilattice tensor}

The following axiom is used in [T06]. It asserts that tensor exists in $\mathcal{C}_{P_{L}}^{o p}$ and is given by product in $\mathcal{C}$. In the case that $\mathcal{C}=$ Loc this is well known since suplattice tensor provides a description of frame coproduct (i.e. of locale product).

Axiom 9. For any objects $X$ and $Y$ of $\mathcal{C}$, the map $\otimes: \mathbb{S}^{X} \times \mathbb{S}^{Y} \rightarrow \mathbb{S}^{X \times Y}$ defined by $\Pi_{\mathbb{S} X \times \mathbb{Y}}\left(\mathbb{S}^{\pi_{1}} \times \mathbb{S}^{\pi_{2}}\right)$ is universal $\sqcup-$ bilinear.

It is not immediate that this axiom extends beyond the binary case for which it is given. Though we would expect, for example, that the map $\otimes$ : $\mathbb{S}^{X} \times \mathbb{S}^{Y} \times \mathbb{S}^{Z} \rightarrow \mathbb{S}^{X \times Y \times Z}$ is necessarily universal $\sqcup-$ trilinear given the axiom, 
the usual proof of this fact does not work because the category $\mathcal{C}_{P_{L}}^{o p}$ is not cartesian closed. However, given Axiom 9, n-ary tensor does exist for $n \geq 2$, but proof of this seems to require a slightly involved lemma which we now provide.

Lemma 7.1.1. Given Axiom 9 and any objects $X, Y$ and $Z$ of $\mathcal{C}$ the map $\otimes: \mathbb{S}^{X} \times \mathbb{S}^{Y} \times \mathbb{S}^{Z} \rightarrow \mathbb{S}^{X \times Y \times Z} \quad$ defined by $\Pi_{\mathbb{S} X \times \mathbb{Y} \times \mathbb{Z}}\left(I d \times \Pi_{\mathbb{S} X \times \mathbb{Y} \times \mathbb{Z}}\right)\left(\mathbb{S}^{\pi_{1}} \times \mathbb{S}^{\pi_{2}} \times \mathbb{S}^{\pi_{3}}\right)$ is universal $\sqcup-$ trilinear.

Proof. Certainly $\otimes: \mathbb{S}^{X} \times \mathbb{S}^{Y} \times \mathbb{S}^{Z} \rightarrow \mathbb{S}^{X \times Y \times Z}$ is join trilinear. We must show that given any join trilinear $\psi: \mathbb{S}^{X} \times \mathbb{S}^{Y} \times \mathbb{S}^{Z} \rightarrow \mathbb{S}^{R}$ that there exists a unique join semilattice homomorphism $\delta: \mathbb{S}^{X \times Y \times Z} \rightarrow \mathbb{S}^{R}$ such that $\delta \otimes=\psi$.

Given any $c: W \times Z \rightarrow \mathbb{S}$ and $W^{\prime}$ an object of $\mathcal{C}$ define

$$
\begin{aligned}
\phi_{W^{\prime}}^{c} & : \quad \mathcal{C}\left(W^{\prime} \times X, \mathbb{S}\right) \times \mathcal{C}\left(W^{\prime} \times Y, \mathbb{S}\right) \rightarrow \mathcal{C}\left(W^{\prime} \times W \times R, \mathbb{S}\right) \\
(a, b) & \mapsto \quad \psi_{W^{\prime} \times W}\left(a \pi_{13}, b \pi_{13}, c \pi_{23}\right)
\end{aligned}
$$

It is routine to verify that this is natural in $W^{\prime}$ and further it is routine to verify that the natural transformation $\phi^{c}: \mathbb{S}^{X} \times \mathbb{S}^{Y} \rightarrow \mathbb{S}^{W \times R}$ so defined is join bilinear. By Axiom 9 there exists a unique join semilattice homomorphism $\gamma^{c}: \mathbb{S}^{X \times Y} \rightarrow \mathbb{S}^{W \times R}$ such that $\gamma^{c} \otimes=\phi^{c}$. By uniqueness note that for any $c_{1}, c_{2}: W \times Z \rightarrow \mathbb{S}, \gamma^{c_{1} \sqcup c_{2}}=\gamma^{c_{1}} \sqcup \gamma^{c_{2}}$ and $\gamma^{0}=0$. Also by uniqueness $\gamma^{c}$ is natural in $c$; if $g: W_{2} \rightarrow W_{1}$ and $c: W_{1} \times Z \rightarrow \mathbb{S}$ then for arbitrary $W^{\prime}$, $\gamma_{W^{\prime}}^{c\left(g \times I d_{Z}\right)}(a \otimes b)$ equals

$$
\begin{aligned}
& \psi_{W^{\prime} \times W_{2}}\left(a \pi_{13}, b \pi_{13}, c\left(g \times I d_{Z}\right) \pi_{23}\right) \\
= & \psi_{W^{\prime} \times W_{2}}\left(a \pi_{13}\left(I d_{W^{\prime}} \times g \times I d_{X}\right), b \pi_{13}\left(I d_{W^{\prime}} \times g \times I d_{Y}\right), c \pi_{23}\left(I d_{W^{\prime}} \times g \times I d_{Z}\right)\right) \\
= & \left(I d_{W^{\prime}} \times g \times I d_{R}\right) \psi_{W^{\prime} \times W_{1}}\left(a \pi_{13}, b \pi_{13}, c \pi_{23}\right) \\
= & \left(I d_{W^{\prime}} \times g \times I d_{R}\right) \gamma_{W^{\prime}}^{c}(a \otimes b)
\end{aligned}
$$

which implies that $\mathbb{S}^{g \times I d_{R}} \gamma^{c} \otimes=\gamma^{c\left(g \times I d_{Z}\right)} \otimes$ and so $\mathbb{S}^{g \times I d_{R}} \gamma^{c}=\gamma^{c\left(g \times I d_{Z}\right)}$ by uniqueness as $\mathbb{S}^{g \times I d_{R}}$ is a join semilattice homomorphism. This verifies our claim that $\gamma^{c}$ is natural in $c$.

We now use $\gamma^{c}$ and its properties to define a join bilinear map $\mathbb{S}^{X \times Y} \times \mathbb{S}^{Z} \rightarrow$ $\mathbb{S}^{R}$ which will therefore extend to our required map $\delta: \mathbb{S}^{X \times Y \times Z} \rightarrow \mathbb{S}^{R}$ by application of Axiom 9. For each object $W$ of $\mathcal{C}$ define a map

$$
\begin{aligned}
& \rho_{W} \quad: \quad \mathcal{C}(W \times X \times Y, \mathbb{S}) \times \mathcal{C}(W \times Z, \mathbb{S}) \rightarrow \mathcal{C}(W \times R, \mathbb{S}) \\
& (I, c) \longmapsto \gamma_{W}^{c}(I) \circ\left(\Delta_{W} \times I d_{R}\right)
\end{aligned}
$$

This is natural in $W$ (use the naturality of $\gamma^{c}$ in $c$ which we have just established). The natural transformation $\rho: \mathbb{S}^{X \times Y} \times \mathbb{S}^{Z} \rightarrow \mathbb{S}^{R}$ so defined is join bilinear since $\gamma^{c}$ is a join semilattice homomorphism and $\gamma^{c_{1}} \sqcup c_{2}=\gamma^{c_{1}} \sqcup \gamma^{c_{2}}$ (and $\gamma^{0}=$ $0)$. By Axiom 9 there exists a join semilattice homomorphism $\delta: \mathbb{S}^{X \times Y \times Z} \rightarrow \mathbb{S}^{R}$ such that $\delta \otimes^{X \times Y, Z}=\rho$ where $\otimes^{X \times Y, Z}: \mathbb{S}^{X \times Y} \times \mathbb{S}^{Z} \rightarrow \mathbb{S}^{X \times Y \times Z}$ is the universal join bilinear tensor. The join trilinear tensor $\otimes: \mathbb{S}^{X} \times \mathbb{S}^{Y} \times \mathbb{S}^{Z} \rightarrow \mathbb{S}^{X \times Y \times Z}$ 
factors as $\otimes: \mathbb{S}^{X} \times \mathbb{S}^{Y} \times \mathbb{S}^{Z} \stackrel{\otimes \times I d}{\longrightarrow} \mathbb{S}^{X \times Y} \times \mathbb{S}^{Z} \stackrel{\otimes^{X \times Y, Z}}{\rightarrow} \mathbb{S}^{X \times Y \times Z}$ and so to complete the existence part of this proof we must just check that

$$
\begin{array}{ccc}
\mathbb{S}^{X} \times \mathbb{S}^{Y} \times \mathbb{S}^{Z} & \stackrel{\otimes \times I d}{\longrightarrow} & \mathbb{S}^{X \times Y} \times \mathbb{S}^{Z} \\
& \psi \searrow & \downarrow \rho \\
& & \mathbb{S}^{R}
\end{array}
$$

commutes. But for each $W$ we have

$$
\begin{aligned}
\rho_{W}(a \otimes b, c) & =\gamma_{W}^{c}(a \otimes b) \circ\left(\Delta_{W} \times I d_{R}\right) \\
& =\psi_{W \times W}\left(a \pi_{13}, b \pi_{13}, c \pi_{23}\right) \circ\left(\Delta_{W} \times I d_{R}\right) \\
& =\psi_{W}\left(a \pi_{13}\left(\Delta_{W} \times I d_{X}\right), b \pi_{13}\left(\Delta_{W} \times I d_{Y}\right), c \pi_{23}\left(\Delta_{W} \times I d_{Z}\right)\right) \\
& =\psi_{W}(a, b, c)
\end{aligned}
$$

by naturality of $\psi$ and so existence is established.

For uniqueness say $\delta^{\prime}: \mathbb{S}^{X \times Y \times Z} \rightarrow \mathbb{S}^{R}$ is also a join semilattice homomorphism with $\delta^{\prime} \otimes=\psi$. For each $c: W \times Z \rightarrow \mathbb{S}$ define $\left(\gamma^{\prime}\right)^{c}: \mathbb{S}^{X \times Y} \rightarrow \mathbb{S}^{W \times R}$ by

$$
\begin{aligned}
& \left(\gamma^{\prime}\right)_{W^{\prime}}^{c} \quad: \quad \mathcal{C}\left(W^{\prime} \times X \times Y, \mathbb{S}\right) \rightarrow \mathcal{C}\left(W^{\prime} \times W \times R, \mathbb{S}\right) \\
& I \longmapsto \delta_{W^{\prime} \times W}^{\prime} \otimes_{W^{\prime} \times W}^{X \times Y, Z}\left(I \pi_{134}, c \pi_{23}\right) .
\end{aligned}
$$

It is routine to verify that this is natural in $W^{\prime}$. The morphism $\left(\gamma^{\prime}\right)^{c}$ so defined is a join semilattice homomorphism since $\delta^{\prime}$ is.

By naturality $\delta_{W}^{\prime} \otimes_{W}^{X \times Y, Z}(I, c)=\left(\gamma^{\prime}\right)_{W}^{c}(I) \circ\left(\Delta_{W} \times I d_{R}\right)$ so the proof will be complete provided we can show that $\left(\gamma^{\prime}\right)^{c}=\gamma^{c}$, since $\delta_{W} \otimes_{W}^{X \times Y, Z}(I, c)=$ $\gamma_{W}^{c}(I) \circ\left(\Delta_{W} \times I d_{R}\right)$ by construction. To show that indeed $\left(\gamma^{\prime}\right)^{c}=\gamma^{c}$ it is sufficient to check that we $\left(\gamma^{\prime}\right)^{c} \otimes=\gamma^{c} \otimes$ i.e. that

$$
\delta_{W^{\prime} \times W}^{\prime} \otimes_{W^{\prime} \times W}^{X \times Y, Z}\left((a \otimes b) \pi_{134}, c \pi_{23}\right)=\psi_{W^{\prime} \times W}\left(a \pi_{13}, b \pi_{13}, c \pi_{23}\right)
$$

for all $a: W^{\prime} \times X \rightarrow \mathbb{S}$ and $b: W^{\prime} \times Y \rightarrow \mathbb{S}$. Since $\delta_{W^{\prime} \times W}^{\prime} \otimes_{W^{\prime} \times W}^{X \times Y, Z}\left(\otimes_{W^{\prime} \times W} \times I d\right)=$ $\psi_{W^{\prime} \times W}$ by assumption (as the trilinear tensor factors as $\left.\otimes_{W^{\prime} \times W}^{X \times Y}\left(\otimes_{W^{\prime} \times W} \times I d\right)\right)$ we therefore are left checking that $\otimes_{W^{\prime} \times W}\left(a \pi_{13}, b \pi_{13}\right)=(a \otimes b) \pi_{134}$. This is a routine unwinding of the definition of the natural transformation $\otimes: \mathbb{S}^{X} \times \mathbb{S}^{Y} \rightarrow$ $\mathbb{S}^{X \times Y}$

$$
\begin{aligned}
\otimes_{W^{\prime} \times W}\left(a \pi_{13}, b \pi_{13}\right) & =\Pi_{\mathbb{S}}\left(a \pi_{123}, b \pi_{124}\right) \\
& =\Pi_{\mathbb{S}}\left(a \pi_{12}, b \pi_{13}\right) \pi_{134} \\
& =\otimes_{W^{\prime}}(a, b) \pi_{134} \\
& =(a \otimes b) \pi_{134} .
\end{aligned}
$$

Proposition 7.1.2. Assuming Axioms 1-4, Axiom 9 is slice stable. 
Proof. If $A, B$ and $C$ are objects of $\mathcal{C} / Y$ then we must check that any join bilinear $\psi: \mathbb{S}_{Y}^{A} \times \mathbb{S}_{Y}^{B} \rightarrow \mathbb{S}_{Y}^{C}$ extends uniquely, via $\otimes: \mathbb{S}_{Y}^{A} \times \mathbb{S}_{Y}^{B} \rightarrow \mathbb{S}_{Y}^{A \times B}$, to a join semilattice homomorphism.

Firstly note that if $A=X_{Y}$ and $B=X_{Y}^{\prime}$ for some objects $X$ and $X^{\prime}$ of $\mathcal{C}$ then the result holds by change of base (from $Y$ to 1 ). But for arbitrary $X_{f}$ an object of $\mathcal{C} / Y$, there exists an equalizer diagram

$$
X_{f} \stackrel{(I d, f)}{\longrightarrow} X_{Y} \stackrel{f \times I d}{\longrightarrow}{ }_{\Delta_{Y} \pi_{2}}^{\longrightarrow} Y_{Y}
$$

in $\mathcal{C} / Y$. The proof will therefore be complete provided we can show that the axiom is stable under taking equalizers. For this it is sufficient to check, given an equalizer diagram $E \stackrel{e}{\hookrightarrow} X \underset{g}{\stackrel{f}{\rightarrow}} Y$ in $\mathcal{C}$ and object $Z$ of $\mathcal{C}$ with $X, Y$ and $Z$ all satisfying the axiom, that $E$ and $Z$ also satisfy the axiom. Say that we are given join bilinear $\psi: \mathbb{S}^{E} \times \mathbb{S}^{Z} \rightarrow \mathbb{S}^{W}$ for some arbitrary object $W$. By the lower coverage theorem join semilattice homomorphisms $\delta: \mathbb{S}^{E \times Z} \rightarrow \mathbb{S}^{W}$ are in order isomorphism with join semilattice homomorphisms $\beta: \mathbb{S}^{X \times Z} \rightarrow \mathbb{S}^{W}$ such that

$$
\beta \sqcap_{\mathbb{S} X \times Z}\left(I d \times \mathbb{S}^{f \times I d_{Z}}\right)=\beta \sqcap_{\mathbb{S} X \times Z}\left(I d \times \mathbb{S}^{g \times I d_{Z}}\right)
$$

since $E \times Z \stackrel{e \times I d_{Z}}{\longrightarrow} X \times Z \underset{g \times I d_{Z}}{\stackrel{f \times I d_{Z}}{\longrightarrow}} Y \times Z$ is an equalizer diagram. In fact, condition (*) is equivalent to $\beta$ composing equally with

$$
\mathbb{S}^{X} \times \mathbb{S}^{Z} \times \mathbb{S}^{Y} \times \mathbb{S}^{Z} \stackrel{\otimes}{\longrightarrow} \mathbb{S}^{X \times Z \times Y \times Z} \stackrel{\mathbb{S}^{\left(\pi_{1}, \pi_{2}, f \pi_{1}, \pi_{2}\right)}}{\longrightarrow} \mathbb{S}^{X \times Z}
$$

and

$$
\mathbb{S}^{X} \times \mathbb{S}^{Z} \times \mathbb{S}^{Y} \times \mathbb{S}^{Z} \stackrel{\otimes}{\longrightarrow} \mathbb{S}^{X \times Z \times Y \times Z} \stackrel{\mathbb{S}^{\left(\pi_{1}, \pi_{2}, g \pi_{1}, \pi_{2}\right)}}{\longrightarrow} \mathbb{S}^{X \times Z}
$$

by the previous lemma where the $\otimes s$ are 4-ary tensors. But (a) factors as

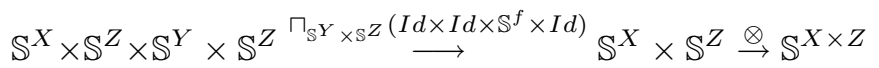

and (b) factors as

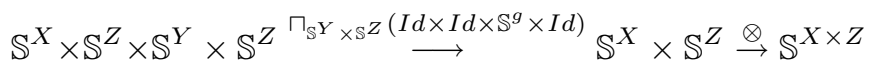

and so since $\mathbb{S}^{e} \times I d: \mathbb{S}^{X} \times \mathbb{S}^{Z} \rightarrow \mathbb{S}^{E} \times \mathbb{S}^{Z}$ composes equally with the first factor of (a') and the first factor of (b') we have that the extension $\overline{\psi\left(\mathbb{S}^{e} \times I d\right)}$ : $\mathbb{S}^{X \times Z} \rightarrow \mathbb{S}^{W}$ of the join bilinear map $\psi\left(\mathbb{S}^{e} \times I d\right): \mathbb{S}^{X} \times \mathbb{S}^{Z} \rightarrow \mathbb{S}^{W}$ must factor via $\mathbb{S}^{X \times Z} \stackrel{\mathbb{S}^{e \times I d} Z}{\rightarrow} \mathbb{S}^{E \times Z}$, and so there exists a join semilattice homomorphism $\delta: \mathbb{S}^{E \times Z} \rightarrow \mathbb{S}^{W}$ with the property that $\delta \mathbb{S}^{e \times I d_{Z}}=\overline{\psi\left(\mathbb{S}^{e} \times I d\right)}$. Certainly the 
diagram

$$
\begin{array}{ccc}
\mathbb{S}^{X \times Z} & \stackrel{\mathbb{S}^{e \times I d} Z}{\longrightarrow} & \mathbb{S}^{E \times Z} \\
\otimes^{X, Z} \uparrow & & \otimes^{E, Z} \uparrow \\
\mathbb{S}^{X} \times \mathbb{S}^{Z} & \stackrel{\mathbb{S}^{e} \times I d}{\longrightarrow} & \mathbb{S}^{E} \times \mathbb{S}^{Z}
\end{array}
$$

commutes and so $\delta \otimes^{E, Z}\left(\mathbb{S}^{e} \times I d\right)=\psi\left(\mathbb{S}^{e} \times I d\right)$ and since, as in the proof of the lower coverage theorem, $\mathbb{S}^{e} \times I d$ is a an epimorphism this shows that $\delta \otimes^{E, Z}=$ $\psi$ as required. The proof is therefore completed with the observation that uniqueness is trivial since (i) $\mathbb{S}^{e \times I d_{z}}$ is an epimorphism and (ii), by assumption, $\otimes^{X, Z}$ is universal.

All of the results of [T06] are therefore slice stable. In particular, applying those techniques to the exposition on subgroupoids contained within Theorem 5.3.1 of [J02], we conjecture that it can shown axiomatically that (i) any subgroupoid $\mathbb{H}$ of $\mathbb{G}$ with open domain and codomain maps is weakly closed over the object object $G_{0}$ of $\mathbb{G}$ and (ii) any subgroupoid $\mathbb{H}$ of $\mathbb{G}$ with proper domain and codomain maps is fitted over the object object $G_{0}$ of $\mathbb{G}$.

\section{References}

\section{References}

[BF96] Bunge, M. and Funk, J. Constructive theory of the lower powerlocale. Math. Structures Comput. Sci. 6 (1996), 69-83.

[HM81] Hofmann, K.H. and Mislove, M.W. Local compactness and continuous lattices. In B. Banaschewski and R.-E. Hoffmann (eds) Continuous Lattices: Proceedings, Bremen 1979, Lecture Notes in Mathematics 871, Springer-Verlag, Berlin (1981), 209-248.

[J82] Johnstone, P.T. Stone Spaces. Cambridge Studies in Advanced Mathematics 3. Cambridge University Press, 1982.

[JV91] Johnstone, P.T., and Vickers, S.J. "Preframe presentations present", in Carboni, Pedicchio and Rosolini (eds) Category Theory - Proceedings, Como, 1990 (Springer Lecture Notes in Mathematics 1488, 1991), 193-212.

[J02] Johnstone, P.T. Sketches of an elephant: A topos theory compendium. Vols 1, 2, Oxford Logic Guides 43, 44, Oxford Science Publications, 2002.

[JT84] Joyal, A. and Tierney, M. An Extension of the Galois Theory of Grothendieck, Memoirs of the American Mathematical Society 309, 1984. 
[Tay00] Taylor, P. Geometric and Higher Order Logic in terms of Abstract Stone Duality. Theory and Applications of Categories 7(15), December 2000, 284-338.

[T04] Townsend, C.F. An Axiomatic account of Weak Localic Triquotient Assignments. Submitted (2003).

[T05] Townsend, C.F. A categorical account of the Hofmann-Mislove theorem. Math. Proc. Camb. Phil. Soc. 139 (2005) 441-456.

[T06] Townsend, C.F. A categorical account of the localic closed subgroup theorem. Comment. Math. Univ. Carolin. 48, 3 (2007) 541-553.

[Vic 89] Vickers. S.J. Topology via Logic. Cambridge Tracts in Theoretical Computer Science, 5. Cambridge University Press, 1989.

[V95] Vickers, S.J. Locales are not pointless, in Hankin, Mackie and Nagarajan (eds) Theory and Formal Methods of Computing 1994 (Imperial College Press, 1995) 199-216.

[V97] Vickers, S.J. Constructive points of powerlocales. Math. Proc. Cam. Phil. Soc. 122 (1997), 207-222.

[VT04] Vickers, S.J. and Townsend, C.F. A Universal Characterization of the Double Power Locale. Theoretical Computer Science 316 (2004) 297-321.

Correspondence: 8 Aylesbury Road, Tring, Hertfordshire, HP23 4DJ, United Kingdom. Email: info@christophertownsend.org 